\title{
TITLE:
}

\section{Off-shell Effect of the Quark-model NN Interaction in the Nd Scattering}

$\operatorname{AUTHOR}(S)$ :

Fujiwara, Yoshikazu; Fukukawa, Kenji

CITATION:

Fujiwara, Yoshikazu ... [et al]. Off-shell Effect of the Quark-model NN Interaction in the Nd Scattering. Few-Body Systems 2012, 12: 2357-2393

ISSUE DATE:

2012-11

URL:

http://hdl.handle.net/2433/169947

\section{RIGHT:}

The final publication is available at www.springerlink.com; This is not the published version. Please cite only the published version.; この論文 は出版社版でありません。引用の際には出版社版をご確認ご利用くだ さい。 
Archive of Applied Mechanics manuscript No.

(will be inserted by the editor)

Y. Fujiwara · K. Fukukawa

\title{
Off-shell effect of the quark-model $N N$ interaction in the $N d$ scattering
}

Received: date / Accepted: date

\begin{abstract}
Low- and medium-energy nucleon-deuteron $(N d)$ scattering less than the incident energy $65 \mathrm{MeV}$ per nucleon is examined by using the quark-model nucleon-nucleon $(N N)$ interaction fss2. The off-shell effect of this non-local interaction yields a part of the attractive effect given by the threenucleon force in the standard description by the meson-exchange potentials. The triton binding energy and the spin-doublet scattering length are well described by fss 2 without introducing the three-nucleon force. The nucleon analyzing power is slightly improved from the results by the AV18 potential, but the so-called $A_{y}$ puzzle in the low-energy region is not essentially solved nor is the similar deficiency of the peak heights seen in the vector analyzing power of the deuteron, $i T_{11}(\theta)$. Other observables are rather similar to the predictions by meson-exchange potentials, including disagreements of the deuteron breakup differential cross sections with experiment for some particular kinematical configurations. The space-star anomaly of the $n d$ and $p d$ scattering at $E_{N}=13 \mathrm{MeV}$ is not improved. The accurate and systematic KVI data of the ${ }^{1} \mathrm{H}(\vec{d}, 2 p) n$ scattering at $E_{d}=130 \mathrm{MeV}$ are compared with the predictions by fss 2 for the breakup differential cross sections and the deuteron analyzing powers.
\end{abstract}

Keywords $n d$ and $p d$ scattering - Quark-model baryon-baryon interaction · AGS equations

\section{Introduction}

The QCD-inspired spin-flavor $S U_{6}$ quark model $(\mathrm{QM})$ for the baryon-baryon interaction, developed by the Kyoto-Niigata group, has achieved accurate description of available nucleon-nucleon $(N N)$ and hyperon-nucleon experimental data [1]. These QM baryon-baryon interactions are characterized by the nonlocality and the energy dependence inherent to the framework of the resonating-group method (RGM) for two three-quark systems. In the strangeness sector, the Pauli forbidden state sometimes exists as the result of the exact antisymmetrization of six quarks. The short-range repulsion of the baryon-baryon interaction is mainly described by the quark-exchange kernel, which gives quite different off-shell properties from the standard meson-exchange potentials. The energy dependence of the interaction is eliminated by the standard off-shell transformation [2], utilizing the square-root inverse of the normalization kernel for the interaction Hamiltonian and the renormalized relative wave function between two clusters. This procedure yields an extra nonlocality, whose effect was examined

Dedicated to Prof. H. Witała on the occasion of his 60 th birthday

Y. Fujiwara

Department of Physics, Kyoto University, Kyoto 606-8502, Japan

Tel.: +81-75-753-3875

Fax: +81-75-753-3866

E-mail: fujiwara_82@msn.com

K. Fukukawa

RIKEN Nishina Center, 2-1 Hirosawa, Wako, Saitama 351-0198, Japan 
in detail for the three-nucleon bound state and for the hypertriton [3]. We found a large triton binding energy by the QM $N N$ interaction; namely, the deficiency of $350 \mathrm{keV}$, predicted by the model fss 2 , is almost half of the standard values $0.5 \mathrm{MeV}-1 \mathrm{MeV}$ [4], given by the modern meson-exchange $N N$ potentials. It is therefore interesting to examine the QM predictions to the three-nucleon scattering, especially in this renormalized framework without the explicit energy dependence of the RGM kernel.

In this study, we apply our QM $N N$ interaction fss2 to the neutron-deuteron ( $n d)$ and protondeuteron $(p d)$ scattering in the Faddeev formalism for systems of composite particles [5]. The AltGrassberger-Sandhas (AGS) equations [6] are solved in the momentum representation, using the off-shell RGM $T$-matrix obtained from the energy-independent renormalized RGM kernel [2]. The Gaussian nonlocal potential constructed from the fss2 is essentially used in the isospin basis [7]. The singularity of the $N N T$-matrix from the deuteron pole is handled in the Noyes-Kowalski method $[8 ; 9]$. Another notorious moving singularity of the free three-body Green function is treated by the standard spline interpolation technique developed by the Böchum-Krakow group [10]. The Coulomb force in the $p d$ scattering is approximately treated by the screened Coulomb force, by introducing a finite cutoff radius $\rho$ [11]. We mainly use the channel-spin formalism which is convenient to discuss the nucleon-deuteron $(N d)$ elastic scattering. The $N N$ interaction up to $I_{\max }=4$ is included in the present calculation, which leads to the nucleon incident energies discussed in this paper up to about $E_{N} \sim 65 \mathrm{MeV}$.

This paper is organized as follows. The QM $N N$ interaction is introduced in the next section, together with the off-shell transformation to eliminate the energy dependence of the RGM kernel. In $\S 3$, the formulation of the present study is summarized, focusing on our treatment of three types of singularities appearing in the AGS equations; the deuteron singularity of the two-nucleon $T$-matrix, the moving singularity of the three-body free Green function, and the Coulomb singularity for the $p d$ scattering. The results of the $n d$ and $p d$ scattering are discussed in $\S 4$ with respect to the eigenphase shifts, effective-range parameters of the $n d$ scattering, total cross sections, elastic differential cross sections, and analyzing powers of the nucleon and the deuteron. The discussion of the deuteron breakup processes in $\S 5$ includes breakup differential cross sections for various kinematical configurations at $E_{N}=13 \mathrm{MeV},{ }^{1} \mathrm{H}(\vec{d}, 2 p) n$ reaction at $E_{d}=16 \mathrm{MeV}$, and comparison with the KVI data for the ${ }^{1} \mathrm{H}(\vec{d}, 2 p) n$ reaction at $E_{d}=130 \mathrm{MeV}$. The final section is devoted to a summary and outlook.

\section{Quark-model baryon-baryon interaction fss2}

The present model is a low-energy effective model which introduces some of the essential features of QCD characteristics. The color degree of freedom of quarks is explicitly incorporated into the nonrelativistic spin-flavor $S U_{6}$ quark model, and the full antisymmetrization of quarks is carried out in the RGM formalism. The gluon exchange effect is represented in the form of the quark-quark interaction. The confinement potential is a phenomenological $r^{2}$-type potential, which has a favorable feature that it does not contribute to the baryon-baryon interactions. We use a color analogue of the Fermi-Breit (FB) interaction, motivated from the dominant one-gluon exchange process in conjunction with the asymptotic freedom of QCD. We postulate that the short-range part of the baryon-baryon interaction is well described by the quark degree of freedom. This includes the short-range repulsion and the spinorbit force, both of which are successfully described by the FB interaction. On the other hand, the medium range attraction and the long-range tensor force, especially afforded by the pions, are extremely non-perturbative from the viewpoint of QCD. These are therefore most relevantly described by the effective meson exchange potentials (EMEP). In the model fss2 [12; 13], the full QM Hamiltonian $H$ consists of the non-relativistic kinetic-energy term, the phenomenological confinement potential $U_{i j}^{\mathrm{Cf}}$, the colored version of the full FB interaction $U_{i j}^{\mathrm{FB}}$ with explicit quark-mass dependence, and the EMEP's $U_{i j}^{\Omega}$ generated from the scalar $(\Omega=\mathrm{S})$, pseudoscalar (PS) and vector (V) meson exchange potentials acting between quarks:

$$
H=\sum_{i=1}^{6}\left(m_{i} c^{2}+\frac{p_{i}^{2}}{2 m_{i}}-T_{G}\right)+\sum_{i<j}^{6}\left(U_{i j}^{\mathrm{Cf}}+U_{i j}^{\mathrm{FB}}+U_{i j}^{\mathrm{S}}+U_{i j}^{\mathrm{PS}}+U_{i j}^{\mathrm{V}}\right)
$$



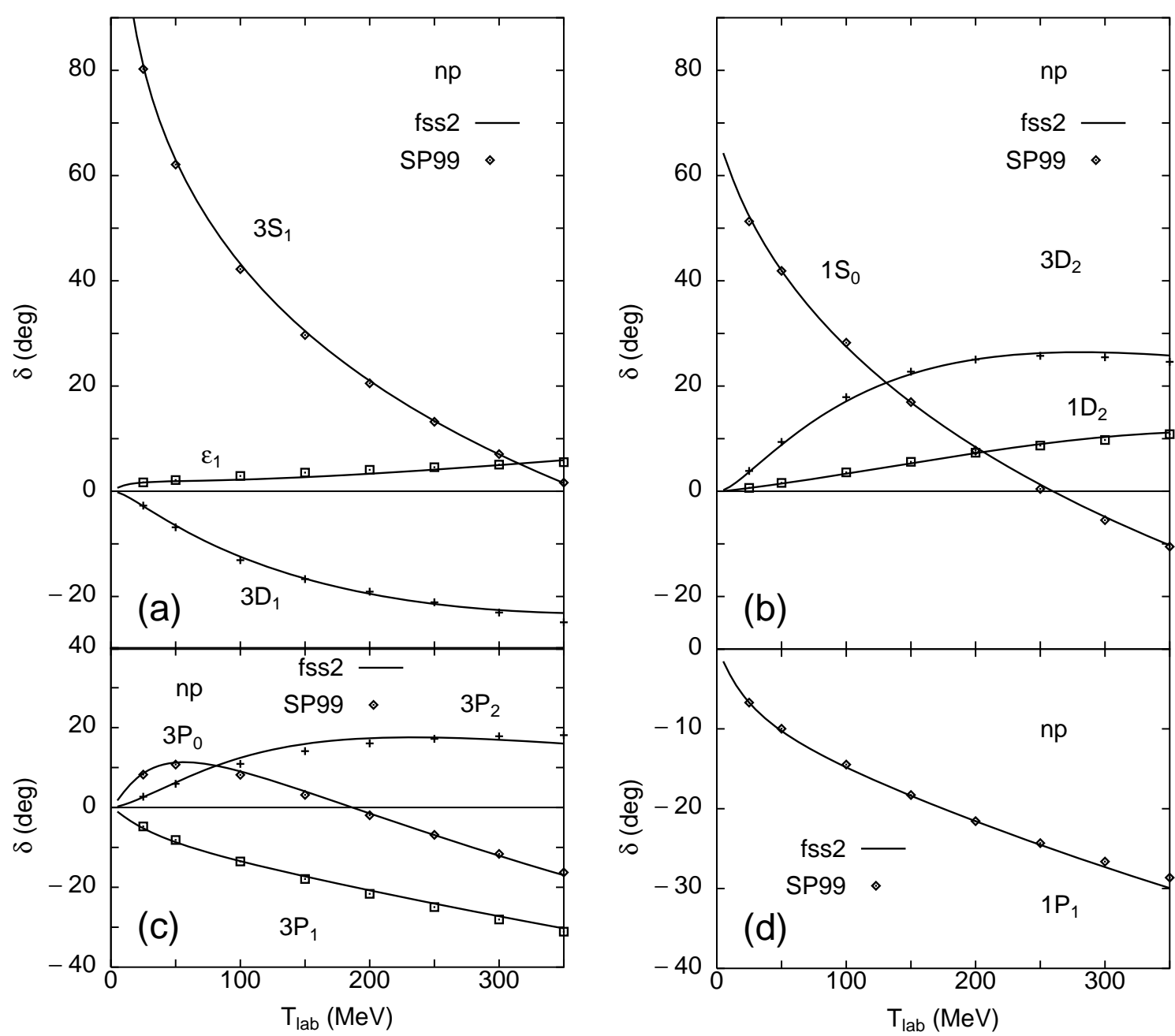

Fig. 1 Calculated $n p$ phase shifts (with $I \leq 2$ ) by fss2 in the isospin basis, compared with the phase-shift analysis SP99 by Arndt et al. [14]

The RGM equation for the relative-motion wave function $\chi(\boldsymbol{r})$ reads

$$
\langle\phi(3 q) \phi(3 q)|E-H| \mathcal{A}\{\phi(3 q) \phi(3 q) \chi(\boldsymbol{r})\}\rangle=0 .
$$

We solve this RGM equation in the momentum representation [15]. If we rewrite the RGM equation in the form of the Schrödinger-type equation as $\left[\varepsilon-H_{0}-V_{\mathrm{RGM}}(\varepsilon)\right] \chi(\boldsymbol{r})=0$, the potential term, $V_{\mathrm{RGM}}(\varepsilon)=V_{\mathrm{D}}+G+\varepsilon K[5]$, becomes nonlocal and energy dependent. Here $V_{\mathrm{D}}$ represents the direct potential of EMEP's, $G$ includes all the exchange kernels for the interaction and kinetic-energy terms, and $K$ is the exchange normalization kernel. Furthermore, $\varepsilon$ is the two-cluster energy in the centerof-mass $(\mathrm{cm})$ system, measured from its threshold. We calculate the plane-wave matrix elements of $V_{\mathrm{RGM}}(\varepsilon)$, and set up with the Lippmann-Schwinger equation of the RGM T-matrix. This approach is convenient to proceed to few-body calculations using the Faddeev formalism [16; 5$]$, and the $G$-matrix calculations as well $[17 ; 18]$. We show in Fig. 1 the $N N$ phase shifts predicted by the model fss 2 . Only the partial waves with the total angular-momentum $I \leq 2$ are shown. More detailed information on the QM baryon-baryon interaction is obtained from the QMPACK homepage [19].

The energy-independent renormalized RGM kernel $V^{\mathrm{RGM}}$ for a two-cluster system reads [2]

$$
V^{\mathrm{RGM}}=V_{\mathrm{D}}+G+W,
$$


where the non-local kernel $W$ appearing through the elimination of the energy-dependence is given by

$$
W=\Lambda \frac{1}{\sqrt{1-K}} h \frac{1}{\sqrt{1-K}} \Lambda-h .
$$

Here, $h$ denotes $h_{0}+V_{\mathrm{D}}+G$ with $h_{0}$ being the kinetic energy for the two-cluster relative motion, and $\Lambda=1-|u\rangle\langle u|$ is a two-cluster Pauli projection operator, where $|u\rangle$ is a Pauli-forbidden state satisfying $K|u\rangle=|u\rangle$. In the $N N$ sector, there appears no Pauli forbidden state at the quark level, so that we can simply set $A=1$ in the following discussion. An advantage of using $V^{\mathrm{RGM}}$, instead of $V_{\mathrm{RGM}}(\varepsilon)$, is that the two-cluster RGM equation takes the form of the usual Schrödinger equation in the Pauli-allowed model space, and the relative wave function is properly normalized. This Schrödingertype equation for the relative wave function gives the same asymptotic behavior as the original RGM equation, thus preserving the phase shifts and physical observables for the two-cluster system. The difference between the previous energy-dependent RGM kernel, $V_{\mathrm{RGM}}(\varepsilon)=V_{\mathrm{D}}+G+\varepsilon K$, and $V^{\mathrm{RGM}}$ in Eq. (2.3) is essentially a replacement of $\Lambda(\varepsilon K) \Lambda$ with $W$. The value of $\varepsilon$ is however not well defined in the three-cluster system, in particular, for scattering systems.

In order to confirm the importance of the present off-shell transformation, we have also calculated the differential cross sections and various polarization observables, using a simple prescription of assuming a constant energy of the two-nucleon subsystem; e.g., the deuteron energy, $\varepsilon=\varepsilon_{d}=-2.2246$ $\mathrm{MeV}$. In this prescription, we come across some disagreements with experimental data. Only when the off-shell transformation is properly taken into account, we can achieve an overall agreement of the scattering observables with the experimental data for the $N d$ elastic scattering. We therefore show throughout this study only the results obtained with the renormalized RGM kernel of the QM $N N$ interaction in Eqs. (2.3) and (2.4) both for the bound-state solution and the scattering problems.

\section{Formulation}

We start with the three-cluster Schrödinger equation,

$$
\left[E-H_{0}-V_{\alpha}^{\mathrm{RGM}}-V_{\beta}^{\mathrm{RGM}}-V_{\gamma}^{\mathrm{RGM}}\right] \Psi=0,
$$

where $V_{\alpha}^{\text {RGM }}$ denotes the energy independent RGM kernel in Eqs. (2.3) and (2.4) with $\Lambda=1$, for the $\alpha$ pair. The subscripts $\alpha, \beta$ and $\gamma$ specify the types of Jacobi coordinates related to the residual pair in the usual way, with $(\alpha, \beta, \gamma)$ being a cyclic permutation of (123). The three-body kinetic energy operator is given by $H_{0}=h_{0}+\bar{h}_{0}$, where $\bar{h}_{0}$ is the kinetic-energy operator for the relative momentum $\boldsymbol{q}=\left(2 \boldsymbol{k}_{3}-\boldsymbol{k}_{1}-\boldsymbol{k}_{2}\right) / 3$. Another momentum for the $N N$ relative motion is denoted by $\boldsymbol{p}=\left(\boldsymbol{k}_{1}-\boldsymbol{k}_{2}\right) / 2$, for which $h_{0}$ is given by $h_{0}=\left(\hbar^{2} / M_{N}\right) p^{2}$ with $M_{N}$ being an average nucleon mass. The vector $\boldsymbol{k}_{i}(i=1-3)$ is the individual momentum of particle $i$. For systems of three-identical particles, the Faddeev equation for the bound state reads [20]

$$
\psi=G_{0} t P \psi,
$$

where $G_{0}=G_{0}(z)=\left(z-H_{0}\right)^{-1}$ is the three-body Green function for the free motion, $P=P_{12} P_{23}+$ $P_{13} P_{23}$ is a sum of the permutation operators for the nucleon rearrangement, and $t=t\left(z-\bar{h}_{0}\right)$ is the $N N T$-matrix derived by solving the standard Lippmann-Schwinger equation $t=v+v G_{0} t$ with $v=V^{\mathrm{RGM}}$. For the time being, we neglect the Coulomb force for simplicity. Furthermore, $z=E$ is the total energy measured from the $3 N$ threshold, which is below the deuteron energy $\varepsilon_{d}(<0)$ for the $3 N$ bound state. A Faddeev component is defined through $\psi=G_{0} v \Psi$, using the total wave function $\Psi=\sum_{\alpha} \psi_{\alpha}$.

The AGS equation describing the $3 N$ scattering is expressed as [20;21]

$$
U|\phi\rangle=G_{0}^{-1} P|\phi\rangle+P t G_{0} U|\phi\rangle,
$$

where the $|\phi\rangle=\left|\boldsymbol{q}_{0}, \psi_{d}\right\rangle$ is the plane-wave channel wave function with $\left|\psi_{d}\right\rangle$ being the normalized deuteron wave function. Here, $z=E+i 0$ is the cm total energy in the complex energy plane and $E$ is expressed as $E=E_{\mathrm{cm}}+\varepsilon_{d}$ with $E_{\mathrm{cm}}=\left(3 \hbar^{2} / 4 M_{N}\right) q_{0}^{2}$ being the nucleon incident energy in the $\mathrm{cm}$ system. The incident energy in the laboratory system is given by $E_{\mathrm{lab}}=(3 / 2) E_{\mathrm{cm}}$ for the nucleonincident reaction and $E_{\mathrm{lab}}=3 E_{\mathrm{cm}}$ for the deuteron-incident reaction with the change of $\boldsymbol{q}_{0} \rightarrow-\boldsymbol{q}_{0}$. 
The scattering amplitude for the elastic scattering is obtained from $\langle\phi|U| \phi\rangle$. It is important that Eq. (3.3) also provides information on the full breakup process of the deuteron. The transition amplitude for the breakup process is given by

$$
U_{0}|\phi\rangle=(1+P) t G_{0} U|\phi\rangle=(1+P) T|\phi\rangle,
$$

where $T=t G_{0} U$ corresponds to the three-body $T$-matrix. The breakup cross sections are obtained from the amplitude $\left\langle\boldsymbol{p} \boldsymbol{q}\left|U_{0}\right| \phi\right\rangle$ with the corresponding on-shell energy $E=\left(\hbar^{2} / M_{N}\right)\left(\boldsymbol{p}^{2}+(3 / 4) \boldsymbol{q}^{2}\right)$. The Faddeev component is expanded into the partial wave components through

$$
|\boldsymbol{p}, \boldsymbol{q} ; 123\rangle=\sum_{\gamma}|p, q, \gamma\rangle\langle\gamma \mid \widehat{\boldsymbol{p}}, \widehat{\boldsymbol{q}} ; 123\rangle
$$

Here, the summation is over all the angular-momentum, spin and isospin quantum numbers in the $\gamma$ channel. We mainly use the channel-spin formalism, for which the two-nucleon total angular-momentum $I$ and the spin $\frac{1}{2}$ of the spectator nucleon is coupled to the channel spin $\left(I \frac{1}{2}\right) S_{c}$. The two-nucleon channel is specified by $(\lambda s) I$; $t$, where $I, \lambda, s$ and $t$ are the total angular momentum, the orbital angular momentum, the spin and the isospin of the $N N$ subsystem, respectively. The $N N$ isospin $t$ is uniquely specified by $\lambda$ and $s$ from the Pauli principle $(-)^{\lambda+s+t}=-1$. The channel spin $S_{c}$ is further coupled with the relative angular momentum $\ell$ between the spectator nucleon and the deuteron, to make the total angular-momentum $\left(\ell S_{c}\right) J$. We specify the deuteron channel with $\lambda=0$ or $2, s=1$, $I=1$ and $t=0$ by $\gamma_{d}$. The channel-spin formalism is particularly convenient to describe the elastic scattering, but the $L S$-coupling scheme is necessary for the breakup processes.

In the scattering problem of the $3 N$ system, we come across three types of singularities, which make the numerical calculation difficult. One is the deuteron singularity in the two-nucleon $T$-matrix, which exists in the deuteron channel $\gamma_{d}$ for the positive incident energy $E_{\mathrm{cm}}>0$. We explicitly separate the delta function part of the deuteron pole through [22]

$$
t=\widetilde{t}-i c G_{0}^{-1}|\phi\rangle\langle\phi| G_{0}^{-1},
$$

where $c=(\pi / 2)\left(4 q_{0} M_{N} / 3 \hbar^{2}\right)$. The difference between $t$ and $\tilde{t}$ appears only when $q=q_{0}$ in the deuteron channel, and $\widetilde{t}$ also satisfies the same basic $T$-matrix equation $\widetilde{t}=v+v G_{0} \widetilde{t}$. The second term in the right-hand side of Eq. (3.6) leads to a mere renormalization of $U_{0}|\phi\rangle$ in Eq. (3.4). We further apply the the Noyes-Kowalski method [8;9] to the resultant equation and obtain a new equation

$$
Q|\phi\rangle=P|\phi\rangle+W \widetilde{t} Q|\phi\rangle
$$

by

$$
G_{0} U|\phi\rangle=Q|\phi\rangle Z^{-1}\langle\phi|U| \phi\rangle
$$

Here, we have defined

$$
W=P G_{0}-P|\phi\rangle Z^{-1}\langle\phi| P \text {. }
$$

and $Z=\left\langle\phi\left|G_{0}{ }^{-1} P\right| \phi\right\rangle$. The rearrangement of the variable $p$ by the permutation $P$ is handled by the spline interpolation technique [20]. We should note that $W$ in Eq. (3.9) satisfies $\langle\phi| G_{0}{ }^{-1} W=0$ and $W G_{0}{ }^{-1}|\phi\rangle=0$. Thus, if we multiply Eq. (3.7) by $\langle\phi| G_{0}{ }^{-1}$ from the left-hand side, we obtain

$$
\left\langle\phi\left|G_{0}{ }^{-1} Q\right| \phi\right\rangle=\left\langle\phi\left|G_{0}^{-1} P\right| \phi\right\rangle=Z,
$$

which is consistent with the definition of $Q|\phi\rangle$ in Eq. (3.8). The basic relationship Eqs. (3.7) and (3.10) implies that $Q|\phi\rangle$ is a modification of $P|\phi\rangle$ by the effect of the nonsingular interaction $\widetilde{t}$, and the real symmetric matrix $Z$ plays an essential role in the following discussion. Furthermore, $\widetilde{t}_{\gamma}$ in Eq. (3.7) can be restored to $t_{\gamma}$ for the deuteron channel $\gamma=\gamma_{d}$ owing to $W G_{0}{ }^{-1}|\phi\rangle=0$ and Eq. (3.6), thus allowing us to write $\widetilde{t}$ as $t$. Because $Z^{-1}$ is a nonsingular matrix, we can define

$$
\widetilde{Q}|\phi\rangle=Q|\phi\rangle Z^{-1}, \quad \widetilde{P}|\phi\rangle=P|\phi\rangle Z^{-1}
$$


We multiply Eq. (3.7) with $Z^{-1}$ from the right-hand side and obtain our final equation

$$
\widetilde{Q}|\phi\rangle=\widetilde{P}|\phi\rangle+W \widetilde{t} \widetilde{Q}|\phi\rangle .
$$

The matrix element $\langle\phi|U| \phi\rangle$ is calculated from

$$
\langle\phi|U| \phi\rangle=\left[Z^{-1}-\langle\phi|X| \phi\rangle+i c \cdot \mathbf{1}\right]^{-1},
$$

where

$$
\langle\phi|X| \phi\rangle=Z^{-1}\langle\phi|P \widetilde{t} Q| \phi\rangle Z^{-1}=\langle\phi|\widetilde{P} \widetilde{t} \widetilde{Q}| \phi\rangle,
$$

is a matrix in the channel-spin variables $\left(\ell S_{c}\right)$ and involves a principal-value integral calculated from the solutions of Eq. (3.12).

In the channel-spin formalism, the asymptotic channel wave function $|\phi\rangle$ is actually $\left|\phi ;\left(\ell S_{c}\right) J J_{z}\right\rangle$ in the partial wave expansion with at most three possible configurations, i.e., $\left(\ell S_{c}\right) J=(J \pm 3 / 2,3 / 2) J$, $(J \mp 1 / 2,1 / 2) J$ and $(J \mp 1 / 2,3 / 2) J$ for the parity $\pi=(-)^{J \mp 1 / 2}$. The partial wave components of the scattering amplitude, $U_{\left(\ell^{\prime} S_{c}^{\prime}\right),\left(\ell S_{c}\right)}^{J}$, are defined by

$\left\langle\phi_{\boldsymbol{q}_{f}} ; S_{c}^{\prime} S_{c z}^{\prime}|U| \phi_{\boldsymbol{q}_{i}} ; S_{c} S_{c z}\right\rangle=\sum_{\ell^{\prime} \ell J J_{z}} U_{\left(\ell^{\prime} S_{c}^{\prime}\right),\left(\ell S_{c}\right)}^{J} \sum_{m^{\prime}}\left\langle\ell^{\prime} m^{\prime} S_{c}^{\prime} S_{c z}^{\prime} \mid J J_{z}\right\rangle Y_{\ell^{\prime} m^{\prime}}\left(\widehat{\boldsymbol{q}}_{f}\right) \sum_{m}\left\langle\ell m S_{c} S_{c z} \mid J J_{z}\right\rangle Y_{\ell m}^{*}\left(\widehat{\boldsymbol{q}}_{i}\right)$,

with $\left|\boldsymbol{q}_{f}\right|=\left|\boldsymbol{q}_{i}\right|=q_{0}$. These are obtained by solving a linear equation derived from Eq. (3.13). The coupled-channel $S$-matrix is related to the scattering amplitudes through

$$
S_{\left(\ell S_{c}\right),\left(\ell^{\prime} S_{c}^{\prime}\right)}^{J}=\delta_{\ell, \ell^{\prime}} \delta_{S_{c}, S_{c}^{\prime}}-2 i c U_{\left(\ell S_{c}\right),\left(\ell^{\prime} S_{c}^{\prime}\right)}^{J} .
$$

Here, the $S$-matrix is a two-dimensional (for $J=1 / 2$ ) or three-dimensional (for $J \neq 1 / 2$ ) matrix. We diagonalize the $S$-matrix by [23]

$$
S=U^{\dagger} e^{2 i \Delta} U
$$

where $\Delta$ is the diagonal matrix whose matrix elements are composed of $e^{i \delta_{\ell S_{c}}^{J}}$. Here, the eigenphase shift $\delta_{\ell S_{c}}^{J}$ is complex for the energies above the deuteron breakup threshold ${ }^{1}$. The unitary matrix $U$ can be parametrized in terms of the mixing parameters $\varepsilon, \xi$ and $\eta$,

$$
\begin{aligned}
U & =\left(\begin{array}{ccc}
1 & 0 & 0 \\
0 & \cos \varepsilon & \sin \varepsilon \\
0 & -\sin \varepsilon & \cos \varepsilon
\end{array}\right)\left(\begin{array}{ccc}
\cos \xi & 0 \sin \xi \\
0 & 1 & 0 \\
-\sin \xi & 0 & \cos \xi
\end{array}\right)\left(\begin{array}{ccc}
\cos \eta & \sin \eta & 0 \\
-\sin \eta \cos \eta & 0 \\
0 & 0 & 1
\end{array}\right) \\
& =\left(\begin{array}{ccc}
\cos \xi \cos \eta & \cos \xi \sin \eta & \sin \xi \\
-\cos \varepsilon \sin \eta-\sin \varepsilon \sin \xi \cos \eta & \cos \varepsilon \cos \eta-\sin \varepsilon \sin \xi \sin \eta & \sin \varepsilon \cos \xi \\
\sin \varepsilon \sin \eta-\cos \varepsilon \sin \xi \cos \eta & -\sin \varepsilon \cos \eta-\cos \varepsilon \sin \xi \sin \eta \cos \varepsilon \cos \xi
\end{array}\right) .
\end{aligned}
$$

We assume $\varepsilon=\xi=0$ for $J^{\pi}=1 / 2^{+}$and $\xi=\eta=0$ for $J^{\pi}=1 / 2^{-}$.

The second singularity appears in $P G_{0}$ in Eq. (3.9) for the positive energies $E>0\left(E_{\mathrm{cm}}>\left|\varepsilon_{d}\right|\right)$. This singularity, usually called a moving singularity from the three-body Green function for the free motion, takes place whenever the breakup process is taken into account. The main idea to avoid this singularity is to apply the spline interpolation to the $q$ variable $[25 ; 10 ; 21 ; 26]$, in addition to the $p$ variable. We first separate the $q-q^{\prime}$ plane into two regions. The one is the region with $q>q_{M}$ or $q^{\prime}>q_{M}$, and the other is $q<q_{M}$ and $q^{\prime}<q_{M}$. Here, $q_{M}$ is the maximum momentum of $q$ for the breakup of the deuteron, given by $q_{M}=\sqrt{q_{0}^{2}-\kappa_{d}^{2}}$ with $\kappa_{d}^{2}$ being related to the deuteron binding energy $\left|\varepsilon_{d}\right|$ through $\left|\varepsilon_{d}\right|=\left(3 \hbar^{2} / 4 M_{N}\right) \kappa_{d}^{2}$. In the region with $q>q_{M}$ or $q^{\prime}>q_{M}, x_{0}$ defined by $x_{0}=x_{0}\left(q, q^{\prime}\right)=\left((3 / 4) q_{M^{2}}-q^{2}-{q^{\prime}}^{2}\right) /\left(q q^{\prime}\right)$ is always less than -1 , so that no singularity appears. In the region with $q<q_{M}$ and $q^{\prime}<q_{M}$, we are confronted with the angular integral,

$$
I\left(q, q^{\prime}\right)=\int_{-1}^{1} d x \frac{F\left(q, q^{\prime} ; x\right)}{x-x_{0}-i 0} .
$$

\footnotetext{
${ }^{1}$ We follow the notation in Ref. [24]. However, it should be noted that $\ell$ and $S_{c}$ are no longer good quantum numbers, but merely characterize the dominant $\ell$ and $S_{c}$ values.
} 
The kernel function $F\left(q, q^{\prime} ; x\right)$ is composed of a combination of spline interpolation functions $G\left(q, q^{\prime} ; x\right)$ and rearrangement coefficients $B\left(q, q^{\prime} ; x\right)$ :

$$
F\left(q, q^{\prime} ; x\right)=G\left(q, q^{\prime} ; x\right) B\left(q, q^{\prime} ; x\right) .
$$

The function $B\left(q, q^{\prime} ; x\right)$ is expressed as the finite sum of the Legendre polynomials of the first kind $P_{k}(x)$.

$$
B\left(q, q^{\prime} ; x\right)=\sum_{k=0}^{k_{M}}(2 k+1) B^{(k)}\left(q, q^{\prime}\right) P_{k}(x),
$$

where $B^{(k)}\left(q, q^{\prime}\right)$ is a polynomial of $q$ and $q^{\prime}$. We modify Eq. (3.19) to

$$
I\left(q, q^{\prime}\right)=\int_{-1}^{1} d x \frac{G\left(q, q^{\prime} ; x\right)-G\left(q, q^{\prime} ; x_{0}\right)}{x-x_{0}} B\left(q, q^{\prime} ; x\right)+G\left(q, q^{\prime} ; x_{0}\right) \int_{-1}^{1} d x \frac{B\left(q, q^{\prime} ; x\right)}{x-x_{0}-i 0} .
$$

In this modification, we have extracted the $x$-dependence of $B\left(q, q^{\prime} ; x\right)$ in the second term of the righthand side. The second term is expressed in terms of the Legendre polynomials of the second kind, $Q_{k}(x) \equiv(1 / 2) \int_{-1}^{1} d t P_{k}(t) /(x-t)$ :

$I\left(q, q^{\prime}\right)=\int_{-1}^{1} d x \frac{G\left(q, q^{\prime} ; x\right)-G\left(q, q^{\prime} ; x_{0}\right)}{x-x_{0}} B\left(q, q^{\prime} ; x\right)+(-2) G\left(q, q^{\prime} ; x_{0}\right) \sum_{k=0}^{k_{M}}(2 k+1) B^{(k)}\left(q, q^{\prime}\right) Q_{k}\left(x_{0}+i 0\right)$,

where

$$
Q_{k}\left(x_{0}+i 0\right)=-\frac{1}{2}\left\{P_{k}\left(x_{0}\right)\left[\log \left|\frac{1-x_{0}}{1+x_{0}}\right|+i \pi \theta\left(1-\left|x_{0}\right|\right)\right]+2 W_{k-1}\left(x_{0}\right)\right\} .
$$

Here, $\theta(x)$ is the step function and $W_{k}(x)$ is a $k$-th degree polynomial of $x$. We need to calculate the integral

$$
\widetilde{I}=\int_{0}^{q_{M}} d q \int_{0}^{q_{M}} d q^{\prime} f(q) I\left(q, q^{\prime}\right) g\left(q^{\prime}\right),
$$

with the spline interpolation

$$
f(q)=\sum_{\mu} S_{\mu}(q) f\left(q_{\mu}\right) \quad \text { for } \quad q \leq q_{M} \quad \text { etc. }
$$

We use the discretization points $q_{\mu}$ in the Gauss-Legendre integration quadrature with $\omega_{\mu}$ being the integration weights. We define the $I_{\mu, \nu}$ through $\widetilde{I}=\sum_{\mu, \nu} f_{\mu} I_{\mu, \nu} g_{\nu}$, where $f_{\mu}$ is defined by $f_{\mu} \equiv$ $\sqrt{\omega_{\mu}} f\left(q_{\mu}\right)$ etc. If $I\left(q, q^{\prime}\right)$ is sufficiently smooth with respect to $q$ and $q^{\prime}$, we can safely apply spline interpolation to $q$ and $q^{\prime}$ variables. We find

$$
\begin{aligned}
I_{\mu, \nu}= & \frac{1}{\sqrt{\omega_{\mu}} \sqrt{\omega_{\nu}}} \int_{0}^{q_{M}} d q \int_{0}^{q_{M}} d q^{\prime} S_{\mu}(q) I\left(q, q^{\prime}\right) S_{\nu}\left(q^{\prime}\right) \\
= & \sqrt{\omega_{\mu}} \sqrt{\omega_{\nu}} \int_{-1}^{1} d x \frac{G\left(q_{\mu}, q_{\nu} ; x\right)-G\left(q_{\mu}, q_{\nu} ; x_{0 \mu \nu}\right)}{x-x_{0 \mu \nu}} B\left(q_{\mu}, q_{\nu} ; x\right) \\
& +\sqrt{\omega_{\mu}} \sqrt{\omega_{\nu}} G\left(q_{\mu}, q_{\nu} ; x_{0 \mu \nu}\right) \sum_{k=0}^{k_{M}}(2 k+1) B^{(k)}\left(q_{\mu}, q_{\nu}\right) \widetilde{Q}_{k \mu \nu},
\end{aligned}
$$

where $x_{0 \mu \nu}=x_{0}\left(q_{\mu}, q_{\nu}\right)$ and

$$
\widetilde{Q}_{k \mu \nu}=(-2) \frac{1}{\omega_{\mu} \omega_{\nu}} \int_{0}^{q_{M}} d q \int_{0}^{q_{M}} d q^{\prime} S_{\mu}(q) Q_{k}\left(x_{0}+i 0\right) S_{\nu}\left(q^{\prime}\right) .
$$

Unfortunately, a complete analytical calculation of the two-side spline interpolation in Eq. (3.28) is not possible. Here, we use the one-side spline interpolation formula with respect to $q^{\prime}$

$$
Q_{k \mu \nu}=(-2) \frac{1}{\omega_{\nu}} \int_{0}^{q_{M}} d q^{\prime} Q_{k}\left(x_{0 \mu}+i 0\right) S_{\nu}\left(q^{\prime}\right)
$$


where $x_{0 \mu}=\left((3 / 4) q_{M}{ }^{2}-q_{\mu}{ }^{2}-{q^{\prime}}^{2}\right) /\left(q_{\mu} q^{\prime}\right)$. The detailed procedure to calculate $Q_{k \mu \nu}$ is given in Appendix B of Ref. [22]. This approximation of the one-side spline interpolation procedure slightly breaks the symmetry with respect to the exchange of $\mu$ and $\nu$. In the actual calculation, we recover the symmetry of the $S$-matrix when we calculate the matrix elements, $X_{\left(\ell S_{c}\right),\left(\ell^{\prime} S_{c}^{\prime}\right)}$ in Eq. (3.14). Namely, we modify $X_{\left(\ell S_{c}\right),\left(\ell^{\prime} S_{c}^{\prime}\right)}$ to $(1 / 2)\left[X_{\left(\ell S_{c}\right),\left(\ell^{\prime} S_{c}^{\prime}\right)}+X_{\left(\ell^{\prime} S_{c}^{\prime}\right),\left(\ell S_{c}\right)}\right]$.

The third singularity is the Coulomb singularity for the $p d$ scattering. The Coulomb $T$-matrix $\left\langle\boldsymbol{p}\left|t^{C}\right| \boldsymbol{p}^{\prime}\right\rangle$ is singular at $\boldsymbol{p}=\boldsymbol{p}^{\prime}$. We avoid this singularity, by using the sharply cut-off Coulomb force introduced at the most basic quark level in the form of $\left(1 / r_{q q}\right) \theta\left(\rho-r_{q q}\right)$, where $r_{q q}$ is the relative distance between two quarks. We use the isospin formalism for introducing the Coulomb force to the AGS equations. The proton-proton $(p p)$ potential $\omega^{\rho}(r ; 1,2)$ is obtained by folding the sharply cut-off Coulomb force with the $(3 q)-(3 q)$ internal wave function, resulting in

$$
\begin{aligned}
\omega^{\rho}(r ; 1,2)= & \frac{e^{2}}{r}\left\{\operatorname{erf}\left(\frac{\sqrt{3}}{2} \frac{r}{b}\right)-\frac{1}{2}\left[\operatorname{erf}\left(\frac{\sqrt{3}}{2} \frac{r+\rho}{b}\right)+\operatorname{erf}\left(\frac{\sqrt{3}}{2} \frac{r-\rho}{b}\right)\right]\right\} \\
& \times \frac{1+\tau_{z}(1)}{2} \frac{1+\tau_{z}(2)}{2},
\end{aligned}
$$

where $r$ is the distance between the two protons, $r=\left|\boldsymbol{r}_{12}\right|=\left|\boldsymbol{x}_{1}-\boldsymbol{x}_{2}\right|$, and $b$ is the harmonic-oscillator range parameter of the (3q)-clusters. The plane-wave matrix elements of Eq. (3.30) is given by

$$
\omega^{\rho}\left(\boldsymbol{p}, \boldsymbol{p}^{\prime} ; 1,2\right)=\left\langle e^{i \boldsymbol{p} \cdot \boldsymbol{r}}\left|\omega^{\rho}(r ; 1,2)\right| e^{i \boldsymbol{p}^{\prime} \cdot \boldsymbol{r}}\right\rangle=e^{2} 2 \pi \rho^{2}\left(\frac{\sin \frac{k \rho}{2}}{\frac{k \rho}{2}}\right)^{2} e^{-\frac{1}{3}(b \boldsymbol{k})^{2}} \frac{1+\tau_{z}(1)}{2} \frac{1+\tau_{z}(2)}{2},
$$

with $k=\left|\boldsymbol{p}-\boldsymbol{p}^{\prime}\right|$, which clearly shows that the Born kernel of the screened Coulomb force is not singular at $\boldsymbol{k}=0$. The angular momentum projection is carried out numerically, using enough number of discretization points for the Gauss-Legendre quadrature. We assume the total isospin $T T_{z}=\frac{1}{2} \frac{1}{2}$ for the ppn system, and neglect the coupling to the isospin $T=\frac{3}{2}$ channel by the Coulomb force. The $T$-matrix is generated from the sum of the nuclear and Coulomb matrix elements for the isospin $t=1$ channels, for which the factor $2 / 3$ is induced from the isospin matrix elements, $\left\langle\gamma\left|\frac{1+\tau_{z}(1)}{2} \frac{1+\tau_{z}(2)}{2}\right| \gamma^{\prime}\right\rangle=\delta_{\gamma, \gamma^{\prime}}(2 / 3)$ for $T=T^{\prime}=1 / 2$.

We calculate the $p d$ screened Coulomb potential by further folding the $p p$ potential in Eq. (3.30) with our deuteron wave function $\left\langle\boldsymbol{r} ; 1,2 \mid \psi_{d}\right\rangle$ :

$$
V_{p d}^{\rho C}(R)=\left\langle\psi^{d}\left|\left[\omega^{\rho}(|\boldsymbol{R}+\boldsymbol{r} / 2| ; 2,3)+\omega^{\rho}(|\boldsymbol{R}-\boldsymbol{r} / 2| ; 3,1)\right]\right| \psi^{d}\right\rangle,
$$

where $\boldsymbol{R}=\boldsymbol{x}_{3}-\left(\boldsymbol{x}_{1}+\boldsymbol{x}_{2}\right) / 2$ is the relative coordinate between the center-of-mass of the deuteron and the proton. This calculation is made in Appendix C of Ref. [11]. We assign the long-range part of Eq. (3.32) to the asymptotic Coulomb potential $W^{\rho}(R)$ for the $p d$ scattering, and parametrize it as $W^{\rho}(R)=\left(e^{2} / R\right) \alpha^{\rho}(R)$. The screening function $\alpha^{\rho}(R)$ is numerically calculated by using the momentum-space deuteron wave function expanded in the dipole form factors [12]. Instead of using the "screening and renormalization" procedure $[27 ; 28 ; 29 ; 30 ; 31 ; 32 ; 33]$, we use an extension of Vincent and Phatak procedure [34] for two-cluster Coulomb problems, which is equivalent to the "screening and renormalization procedure" only in the limit of $\rho \rightarrow \infty$. The scattering amplitude is obtained by imposing a connection condition on the $K$-matrix $K_{\alpha, \beta}^{\rho} \equiv\left(Z^{-1}\right)_{\alpha, \beta}-\left\langle\phi_{\alpha}\left|X^{\rho}\right| \phi_{\beta}\right\rangle$ for the $p d$ scattering [22], which is derived from two different asymptotic forms of the reduced wave function $\Phi_{\alpha, \gamma}^{\rho(+)}(R) \equiv\left\langle R, \psi_{\alpha}^{d} \mid \Psi_{\gamma}^{\rho(+)}\right\rangle$. Here, the subscripts $\alpha, \gamma$, etc. specify the channel quantum numbers. The first one is the approximate Coulomb wave for the relative distance of two clusters, $r$, smaller than $R_{\text {in }}(<\rho)$, and the other is free (no-Coulomb) wave in the longer range region, $r>R_{\text {out }}>\rho$. Here, $R_{\text {in }}$ should be sufficiently large in comparison with the range of the nuclear force. The asymptotic Hamiltonian composed of the screened Coulomb force allows us to calculate constant Wronskians of this Hamiltonian in either region, in terms of the modified Coulomb wave functions $\widetilde{F}_{\ell}^{\rho}\left(q_{0}, R\right)$ and $\widetilde{G}_{\ell}^{\rho}\left(q_{0}, R\right)$. Using this property, we can extend the standard procedure of matching conditions for asymptotic waves to the screened Coulomb potentials. The connection condition for $\Phi_{\alpha, \gamma}^{\rho(+)}(R)$ at 
$R=R_{\text {out }}$ is written in terms of Wronskians:

$$
\begin{aligned}
& \sum_{\beta} \widetilde{K}_{\alpha, \beta}^{\rho}\left\{W\left[\widetilde{F}_{\beta}^{\rho}, u_{\beta}\right]_{R_{\text {out }}} K_{\beta, \gamma}^{\rho}-W\left[\widetilde{F}_{\beta}^{\rho}, v_{\beta}\right]_{R_{\text {out }}} c \delta_{\beta, \gamma}\right\} \\
& =c\left\{W\left[\widetilde{G}_{\alpha}^{\rho}, u_{\alpha}\right]_{R_{\text {out }}} K_{\alpha, \gamma}^{\rho}-W\left[\widetilde{G}_{\alpha}^{\rho}, v_{\alpha}\right]_{R_{\text {out }}} c \delta_{\alpha, \gamma}\right\} .
\end{aligned}
$$

The matrix elements $\widetilde{U}_{\beta, \gamma}^{\rho}$ defined by

$$
\sum_{\beta}\left[\widetilde{K}_{\alpha, \beta}^{\rho}+i c \delta_{\alpha, \beta}\right] \widetilde{U}_{\beta, \gamma}^{\rho}=\delta_{\alpha, \gamma}
$$

in the $\rho \rightarrow \infty$ limit are related to the scattering amplitude of the Coulomb-modified AGS equation with respect to the Coulomb distorted waves for the screening Coulomb potential. For details, Ref. [11] should be referred to. The scattering amplitude $f_{\beta, \gamma}^{N, \rho}$ is obtained from $\widetilde{U}_{\beta, \gamma}^{\rho}$ through

$$
f_{\beta, \gamma}^{N, \rho}=-\frac{\pi}{2} \frac{4 M_{N}}{3 \hbar^{2}} \widetilde{U}_{\beta, \gamma}^{\rho}
$$

In the channel-spin formalism, the full scattering amplitude is written as

$$
\begin{aligned}
& f_{S_{c}^{\prime} S_{c z}^{\prime}, S_{c} S_{c z}}^{\rho}\left(\widehat{\boldsymbol{q}}_{f}, \widehat{\boldsymbol{q}}_{i}\right)=\delta_{S_{c}^{\prime}, S_{c}} \delta_{S_{c z}^{\prime}, S_{c z}} f^{C}(\theta)+4 \pi \sum_{\ell^{\prime} \ell J J_{z}} e^{i\left(\sigma_{\ell^{\prime}}+\sigma_{\ell}\right)} f_{\left(\ell^{\prime} S_{c}^{\prime}\right),\left(\ell S_{c}\right)}^{N J, \rho} \\
& \times \sum_{m^{\prime}}\left\langle\ell^{\prime} m^{\prime} S_{c}^{\prime} S_{c z}^{\prime} \mid J J_{z}\right\rangle Y_{\ell^{\prime} m^{\prime}}\left(\widehat{\boldsymbol{q}}_{f}\right) \sum_{m}\left\langle\ell m S_{c} S_{c z} \mid J J_{z}\right\rangle Y_{\ell m}^{*}\left(\widehat{\boldsymbol{q}}_{i}\right),
\end{aligned}
$$

for a sufficiently large $\rho$. In Eq. (3.36), $f^{C}(\theta)$ is the Rutherford amplitude and $\sigma_{\ell}$ etc. are the Coulomb phase shifts for the partial wave $\ell$. For the $p d$ scattering, the definition of the eigenphase shift in Eqs. (3.17) and (3.18) is applied to the nuclear part $\widetilde{U}_{\beta, \gamma}^{\rho}$ in Eq. (3.35).

The $3 N$ breakup kinematics is completely specified by the three-dimensional momenta of the three particles. Among them, only $5(=3 \times 3-4)$ quantities are independent owing to the energy- and momentum-conservation laws. Assuming the experimental setup to detect two particles 1 and 2 , we choose these as the two polar angles $\theta_{1}$ and $\theta_{2}$, and the difference of the azimuthal angles $\phi_{12}=\phi_{1}-\phi_{2}$ for two vectors $\boldsymbol{k}_{1}$ and $\boldsymbol{k}_{2}$, in addition to the energy $S$ determined from $k_{1}=\left|\boldsymbol{k}_{1}\right|$ and $k_{2}=\left|\boldsymbol{k}_{2}\right|$ by the energy conservation. We also choose the $z$-axis as the direction of the incident particle and the $x$-axis such that $\phi_{1}=\pi$ [35]. For a specific total energy (or a nucleon or deuteron incident energy), the energies of particle 1 and $2, E_{i}=\left(\hbar^{2} / 2 M_{N}\right) k_{i}^{2}(i=1,2)$, are specified by a locus of the $E_{1}-E_{2}$ energy plane. We call this a kinematical curve. Along the kinematical curve in the $E_{1}-E_{2}$ plane, the arc length $S$ is measured starting from a certain starting point. The $3 N$ breakup kinematics used in this paper is summarized in Appendix A of Ref. [36], including the transformations between the $\mathrm{cm}$ and lab. systems. The breakup differential cross sections of the $N d$ scattering in the cm system are calculated from

$$
\frac{d^{5} \sigma}{d \widehat{\boldsymbol{p}} d \widehat{\boldsymbol{q}} d q}=(2 \pi)^{4}\left(\frac{2 M_{N}}{3 \hbar^{2}}\right)^{2} \frac{3}{4} \frac{p_{0} q^{2}}{q_{0}} \frac{1}{6} \sum_{\Gamma} \sum_{S_{c} S_{c z}}\left|\left\langle\boldsymbol{p q} \Gamma\left|\mathcal{O}_{\tau}(1+P) T\right| \phi_{\boldsymbol{q}_{0}} ; S_{c} S_{c z}\right\rangle_{0}\right|^{2}
$$

where $\Gamma=\Gamma_{\sigma} \Gamma_{\tau}$ is the spin-isospin quantum numbers in the $L S$-coupling scheme and the subscript 0 in the matrix element implies the on-shell condition $|\boldsymbol{p}|=p_{0}=\sqrt{(3 / 4)\left(q_{M^{2}}-q^{2}\right)}$ with $q_{M}$ being the maximum momentum of $q$ for the deuteron breakup. The species of the detected particles in the final state are controlled by the isospin projection operator $\mathcal{O}_{\tau}$; i.e.,

$$
\mathcal{O}^{p p}=\frac{1+\tau_{z}(1)}{2} \frac{1+\tau_{z}(2)}{2}, \quad \mathcal{O}^{n n}=\frac{1-\tau_{z}(1)}{2} \frac{1-\tau_{z}(2)}{2}
$$

for the two protons and two neutrons, respectively. The permutation operator $P$ in Eq. (3.37) acts on the momentum space and the spin-isospin space. In dealing with the permutation operator in the 
momentum space, we operate $P$ on the bra side and use all the Jacobi coordinates $\boldsymbol{p}_{\alpha}$ and $\boldsymbol{q}_{\alpha}(\alpha=1,2$, and 3) to avoid the slow convergence of the partial-wave expansion with respect to the rearrangement factors. From this process, we obtain

$$
\begin{aligned}
& \frac{d^{5} \sigma}{d \widehat{\boldsymbol{p}} d \widehat{\boldsymbol{q}} d q}=\frac{3}{4} \frac{p_{0} q^{2}}{q_{0}} \frac{1}{6} \sum_{S_{c}, S_{c z}}\left[\sum_{\alpha=1}^{3} \sum_{\widetilde{\Gamma}, \Gamma} \delta_{\tilde{S}, S} \delta_{\tilde{s}, s} X_{\tilde{t}, t}^{\tau(\alpha \alpha)} f_{\widetilde{\Gamma}, S_{c} S_{c z}}^{*}\left(\boldsymbol{p}_{\alpha}, \boldsymbol{q}_{\alpha}\right) f_{\Gamma, S_{c} S_{c z}}\left(\boldsymbol{p}_{\alpha}, \boldsymbol{q}_{\alpha}\right)\right. \\
& \left.+\sum_{(\alpha \beta \gamma)}^{\prime} \sum_{\widetilde{\Gamma}, \Gamma} \delta_{\tilde{S}, S}(-2) X_{\tilde{s}, s}^{S} X_{\tilde{t}, t}^{\tau(\alpha \beta)} \operatorname{Re}\left\{f_{\widetilde{\Gamma}, S_{c} S_{c z}}^{*}\left(\boldsymbol{p}_{\alpha}, \boldsymbol{q}_{\alpha}\right) f_{\Gamma, S_{c} S_{c z}}\left(-\boldsymbol{p}_{\beta}, \boldsymbol{q}_{\beta}\right)\right\}\right]
\end{aligned}
$$

where $X_{\tilde{s}, s}^{S}$ and $X_{\tilde{t}, t}^{\tau(\alpha \beta)}$ etc. are the spin-isospin factors given in Appendix B of Ref. [36], and the direct breakup amplitude is calculated from

$$
\begin{aligned}
f_{\Gamma, S_{c} S_{c z}}(\boldsymbol{p}, \boldsymbol{q})= & \sqrt{4 \pi} \sum_{\gamma, \ell^{\prime}, J}^{\prime}\left\{\begin{array}{c}
1 \\
(-1)^{\ell^{\prime}}
\end{array}\right\} f_{\gamma,\left(\ell^{\prime} S_{c}\right)}^{(\mathrm{db}) J}(q)\left\langle L\left(S_{c z}-S_{z}\right) S S_{z} \mid J S_{c z}\right\rangle \\
& \times \widehat{\ell}^{\prime}\left\langle\ell^{\prime} 0 S_{c} S_{c z} \mid J S_{c z}\right\rangle Y_{(\lambda \ell) L\left(S_{c z}-S_{z}\right)}(\widehat{\boldsymbol{p}}, \widehat{\boldsymbol{q}}) \quad \text { for } \quad \begin{array}{c}
\text { nucleon-incident } \\
\text { deuteron-incident }
\end{array},
\end{aligned}
$$

with $\widehat{\ell}^{\prime}=\sqrt{2 \ell^{\prime}+1}$ and

$$
f_{\gamma,\left(\ell S_{c}\right)}^{(\mathrm{db}) J}(q)=\int_{0}^{\infty} p^{2} d p\left\langle p_{0}\left|t_{\gamma}\left(\hbar^{2} p_{0}^{2} / M_{N}\right)\right| p\right\rangle \sum_{\left(\ell^{\prime} S_{c}^{\prime}\right)}\left\langle p, q, \gamma|\widetilde{Q}| \phi ;\left(\ell^{\prime} S_{c}^{\prime}\right) J\right\rangle f_{\left(\ell^{\prime} S_{c}^{\prime}\right),\left(\ell S_{c}\right)}^{J}
$$

with the elastic scattering amplitude, $f_{\left(\ell^{\prime} S_{c}^{\prime}\right),\left(\ell S_{c}\right)}^{J}$, similar to Eq. (3.35). The breakup differential cross sections in the lab system, $\left(d^{5} \sigma / d \widehat{\boldsymbol{k}}_{1} d \widehat{\boldsymbol{k}}_{2} d S\right)$, are specified by the two directions $\widehat{\boldsymbol{k}}_{1}$ and $\widehat{\boldsymbol{k}}_{2}$, and the energy $S$ measured along the locus of the $E_{1}-E_{2}$ energy plane. They are obtained from the right-hand side of Eq. (3.39) by simply changing the phase space factor [21]

$$
\begin{aligned}
\rho_{\mathrm{cm}}=\frac{3}{4} \frac{p_{0} q^{2}}{q_{0} \longrightarrow \rho_{\mathrm{lab}}=} & \frac{M_{N}}{\hbar^{2}} \frac{3 k_{1} k_{2}}{2 q_{0}}\left[\left(2-\frac{k_{\mathrm{lab}}}{k_{2}} \cos \theta_{2}+\frac{k_{1}}{k_{2}} \cos \theta_{12}\right)^{2}\right. \\
& \left.+\left(2-\frac{k_{\mathrm{lab}}}{k_{1}} \cos \theta_{1}+\frac{k_{2}}{k_{1}} \cos \theta_{12}\right)^{2}\right]^{-1 / 2}
\end{aligned}
$$

Here, $k_{\text {lab }}$ is the incident lab. momentum and $\theta_{12}$ is the relative angle between $\boldsymbol{k}_{1}$ and $\boldsymbol{k}_{2}$. The direct breakup amplitudes for the deuteron-incident reaction are generated by adding an extra phase factor $(-1)^{\ell^{\prime}}$ to each term as in Eq. (3.40), corresponding to the change from $\widehat{\boldsymbol{q}}_{0}$ to $-\widehat{\boldsymbol{q}}_{0} \cdot{ }^{2}$

The deuteron analyzing powers of the breakup differential cross sections are calculated from modifying the initial channel-spin sum in Eq. (3.39) to the spin-transition by $\left\langle S_{c}^{\prime} S_{c z}^{\prime}\left|S_{\mu}^{(\lambda)}\right| S_{c} S_{c z}\right\rangle$. Here, $S_{\mu}^{(\lambda)}$ with $\lambda=1$ and 2 are the spin operators of the deuteron given in Appendix A of Ref. [37]. We denote these spin-transfer differential cross sections by $I(\lambda \mu)$. Then, the vector-type and tensor-type deuteron analyzing powers are given by

$$
\begin{aligned}
& A_{x}(\xi)=\frac{1}{\sqrt{2}}[-I(11)+I(11)], \quad A_{y}(\xi)=i \frac{1}{\sqrt{2}}[I(11)+I(11)] \\
& A_{x y}(\xi)=-i \frac{3}{2}[I(22)+I(2,-2)], \quad A_{x x}(\xi)=-\sqrt{\frac{3}{2}} I(20)+\frac{3}{2}[-I(22)+I(2,-2)] \\
& A_{y y}(\xi)=-\sqrt{\frac{3}{2}} I(20)-\frac{3}{2}[-I(22)+I(2,-2)]
\end{aligned}
$$

for each particular breakup kinematical configuration $\xi$.

\footnotetext{
${ }^{2}$ We thank Professor H. Witała for notifying us about this phase change.
} 
For the $d p$ and $p d$ reactions, we incorporate the screened Coulomb force in a way similar to the $p d$ elastic scattering. The treatment of the half off-shell $T$-matrix in Eq. (3.41) for the screened Coulomb potential is only different from the elastic scattering. When the $p p$ pair is detected, $t^{I=1}=t^{p p}$ is adopted as the half off-shell $T$-matrix for the $\alpha=3$ amplitude. The $\alpha=1$ and $\alpha=2$ amplitudes are generated from $t^{n p}$ both for $I=0$ and $I=1$ channels. The Coulomb-modified breakup amplitude is generated from the large- $\rho$ limit of the breakup amplitude including the screened Coulomb force; i.e.,

$$
\begin{aligned}
& \left\langle\boldsymbol{q}, \psi_{\boldsymbol{p}}^{(-)}\left|\widetilde{U}_{0}\right| \psi^{(+)}\right\rangle_{0}=\lim _{\rho \rightarrow \infty} e^{i z^{\rho}(p)}\left\langle\boldsymbol{q}, \psi_{\boldsymbol{p}}^{\rho(-)}\left|\widetilde{U}_{0}^{\rho}\right| \psi^{\rho(+)}\right\rangle_{0} e^{i \zeta\left(q_{0}\right)}, \\
& \sim e^{i z^{\rho}(p)}\left\langle\boldsymbol{p}, \boldsymbol{q}\left|(1+P) t^{\rho} G_{0} U^{\rho}\right| \phi\right\rangle_{0} e^{i \zeta\left(q_{0}\right)} .
\end{aligned}
$$

Since the renormalization phases, $z^{\rho}(p)$ and $\zeta\left(q_{0}\right)$, are $\lambda$ - or $\ell$-independent in the limit of $\rho \rightarrow \infty$, we neglect these phase factors in the actual calculations.

\section{$4 N d$ elastic scattering}

\subsection{Eigenphase shifts of $n d$ and $p d$ scattering}

Table 1 shows the $n d$ and $p d$ eigenphase shifts, predicted by fss 2 , at the incident energy $E_{N}=3 \mathrm{MeV}$ below the deuteron breakup threshold. In this and all other calculations of the elastic scattering, the maximum angular momentum for the $N N$ system is taken up to $I_{\max }=4$, and the momentum meshpoints $n=6-6-5$ in the definition discussed in $\S 3.1$ of Ref. [22] are used, unless otherwise specified. The Coulomb cutoff radius $\rho=9 \mathrm{fm}(\rho=8 \mathrm{fm})$ is assumed for the elastic scattering, depending on the incident energies below (above) the deuteron breakup threshold. Table 1 also shows predictions by the Pisa group using AV18 and AV18 plus Urbana (UR) $3 N$ potentials [38], as well as the pd eigenphase shifts by the phase-shift analysis (PSA) [38] based on the AV18 + UR3N potentials. From Table 1, we find an outstanding feature that the UR $3 N$ force has a sizable effect only in the $J^{\pi}=1 / 2^{+}$channel. We also find that our results by fss 2 are very similar not to AV18 but to the AV18+UR $3 N$ shown in the parentheses. Our $n d$ and $p d^{2} S_{1 / 2}$ nuclear phase shifts are more attractive than AV18 by $3^{\circ}-5^{\circ}$ at the range of $E_{N}=1-3 \mathrm{MeV}$. In the same energy range, the maximum difference from the AV18+UR3N results is only $0.6^{\circ}$. This attractive behavior is not surprising since the model fss 2 predicts the almost correct triton binding energy. In spite of the attractive tendency in the ${ }^{2} S_{1 / 2}$ channel, we find from Table 1 that our ${ }^{2} S_{1 / 2}$ nuclear phase shift at $E_{p}=3 \mathrm{MeV}$ is not so attractive as to reproduce the result of PSA. This is apparently because the binding energy of ${ }^{3} \mathrm{H}$ is still insufficiently reproduced even for fss2. The model fss 2 also gives the mixing parameter $\eta_{1 / 2}^{+}$very similar to that by AV18+UR3N . For other channels than ${ }^{2} S_{1 / 2}$, the difference of the eigenphase shifts from the AV18 or AV18+UR3N results is less than $1^{\circ}$. After all, we have found a good correspondence of the nuclear phase shifts below the deuteron breakup threshold between our fss 2 and the AV18+UR3N potentials. It is reported that the attractive behavior of UR $3 N$ force is important for good starting point for PSA (See Fig. 10 of Ref. [39]). In our case this attractive behavior is related to the strong deuteron distortion effect, which is sensitive to the description of the short-range $N N$ repulsion.

As to the ${ }^{4} S_{3 / 2}$ channel, the eigenphase shifts are strongly repulsive due to the Pauli principle, and a good correspondence between fss 2 and AV18 or AV18+UR3N results is seen in Table 1 . The $p d$ phase shift of fss2 is also similar to that of the PSA. We have examined the energy dependence of the $n d$ and $p d$ eigenphase shifts up to $E_{N}=50 \mathrm{MeV}$, with respect to the low partial waves. The ${ }^{4} S_{3 / 2}$ eigenphase shifts determined by the PSA are very similar to those by fss 2 at $E_{p} \leq 14 \mathrm{MeV}$ and tend to deviate from the fss2 values at higher energies, to more repulsive direction. This tendency is also seen in the calculation using AV18 [39]. The imaginary part is generally much larger in the doublet scattering than in the quartet scattering, which is also true for the $P$ - and $D$-waves. This result implies that the deuteron breakup effect in the doublet channels is stronger than in the quartet channels. For the $P$-wave phase shifts, ${ }^{4} P_{J}$ phase shifts show the attractive behaviour with rather monotonic rise up to $30^{\circ}-40^{\circ}$ at $E_{N}=20 \mathrm{MeV}$, but two ${ }^{2} P_{J}$ phase shifts with $J=3 / 2$ and $1 / 2$ change the sign around $E_{N}=10-20 \mathrm{MeV}$ from negative to positive. These behaviors are very similar to the AV18 predictions in Ref. [39] and to the PSA on the whole. It is well known that the nucleon analyzing power $A_{y}(\theta)$ at the maximum point is very sensitive to the slight change of the ${ }^{4} P_{J}$ eigenphase shifts [40]. In our 
Table 1 The $n d$ and $p d$ eigenphase shifts at $E_{N}=3 \mathrm{MeV}$, predicted by fss 2 . The maximum angular momentum for the $N N$ system, $I_{\max }=4$, and the momentum mesh points $n=6-6-5$ are used. For the $p d$ calculation, the cutoff Coulomb radius $\rho=9 \mathrm{fm}$ is used. The corresponding parameters calculated by the Pisa group from the AV18 potential models are also listed for comparison [38]. The parameters in the parentheses are predictions by the AV18+UR3N potentials. Results of the phase shift analysis (PSA) [38] are based on the calculations using the AV18+UR3N potentials.

\begin{tabular}{|c|c|c|c|c|c|}
\hline Model & $\overline{f s s 2(n d)}$ & AV18(+UR3N) $(n d)$ & $\overline{f s s 2(p d)}$ & AV18(+UR3N) $(p d)$ & $\overline{\mathrm{PSA}}$ \\
\hline${ }^{4} D_{1 / 2}$ & -3.82 & $-3.85(-3.84)$ & -3.36 & $-3.56(-3.57)$ & $-3.38 \pm 0.01$ \\
\hline${ }^{2} S_{1 / 2}$ & -30.8 & $-35.3(-30.8)$ & -27.2 & $-32.2(-27.8)$ & $-24.85 \pm 0.23$ \\
\hline$\eta_{1 / 2+}$ & 1.55 & $1.12(1.45)$ & 1.60 & $1.10(1.47)$ & $1.65 \pm 0.02$ \\
\hline${ }^{2} P_{1 / 2}$ & -7.44 & $-7.49(-7.50)$ & -7.05 & $-7.36(-7.37)$ & $-7.41 \pm 0.08$ \\
\hline${ }^{4} P_{1 / 2}$ & 23.3 & $24.2(24.5)$ & 21.7 & $22.1(22.3)$ & $21.77 \pm 0.01$ \\
\hline$\varepsilon_{1 / 2-}$ & 6.77 & $6.68(6.82)$ & 5.85 & $5.71(5.83)$ & $5.70 \pm 0.05$ \\
\hline${ }^{4} S_{3 / 2}$ & -69.6 & $-69.9(-69.7)$ & -62.5 & $-63.1(-63.1)$ & $-63.80 \pm 0.11$ \\
\hline${ }^{2} D_{3 / 2}$ & 2.34 & $2.36(2.36)$ & 2.08 & $2.15(2.15)$ & $2.23 \pm 0.02$ \\
\hline${ }^{4} D_{3 / 2}$ & -4.12 & $-4.14(-4.14)$ & -3.63 & $-3.83(-3.83)$ & $-3.76 \pm 0.01$ \\
\hline$\varepsilon_{3 / 2+}$ & 0.763 & $0.747(0.754)$ & 0.866 & $0.800(0.802)$ & $0.975 \pm 0.060$ \\
\hline$\xi_{3 / 2+}$ & 1.36 & $1.35(1.35)$ & 1.31 & $1.30(1.30)$ & $1.27 \pm 0.01$ \\
\hline$\eta_{3 / 2+}$ & -0.364 & -0.363 & -0.319 & $-0.322(-0.316)$ & $-0.257 \pm 0.09$ \\
\hline${ }^{4} F_{3 / 2}$ & 0.919 & $0.920(0.921)$ & 0.775 & $0.800(0.802)$ & $0.928 \pm 0.005$ \\
\hline${ }^{2} P_{3 / 2}$ & -7.10 & $-7.18(-7.20)$ & -6.80 & $-7.14(-7.15)$ & $-7.18 \pm 0.04$ \\
\hline${ }^{4} P_{3 / 2}$ & 25.5 & $26.0(26.0)$ & 24.0 & $24.2(24.2)$ & $24.30 \pm 0.01$ \\
\hline$\varepsilon_{3 / 2-}$ & -2.71 & $-2.61(-2.66)$ & -2.32 & $-2.20(-2.23)$ & $-2.46 \pm 0.01$ \\
\hline$\xi_{3 / 2-}$ & -0.427 & $-0.265(-0.256)$ & -0.427 & $-0.321(-0.314)$ & $-0.352 \pm 0.049$ \\
\hline$\eta_{3 / 2-}$ & -3.57 & $-3.52(-3.53)$ & -3.19 & $-3.11(-3.11)$ & $-3.24 \pm 0.05$ \\
\hline${ }^{4} G_{5 / 2}$ & -0.206 & -0.206 & -0.172 & -0.189 & - \\
\hline${ }^{2} D_{5 / 2}$ & 2.31 & 2.33 & 2.05 & 2.13 & $2.18 \pm 0.02$ \\
\hline${ }^{4} D_{5 / 2}$ & -4.44 & -4.46 & -3.92 & -4.13 & $-4.07 \pm 0.02$ \\
\hline$\varepsilon_{5 / 2+}$ & -0.313 & -0.315 & -0.345 & -0.350 & $-0.265 \pm 0.034$ \\
\hline$\xi_{5 / 2+}$ & -0.716 & -0.701 & -0.710 & -0.699 & - \\
\hline$\eta_{5 / 2+}$ & -2.02 & -2.04 & -2.02 & -2.07 & - \\
\hline${ }^{4} P_{5 / 2}$ & 25.8 & $26.0(26.3)$ & 23.8 & $23.9(24.1)$ & $24.26 \pm 0.01$ \\
\hline${ }^{2} F_{5 / 2}$ & -0.465 & -0.466 & -0.396 & -0.433 & $-0.485 \pm 0.026$ \\
\hline${ }^{4} F_{5 / 2}$ & 0.947 & 0.951 & 0.800 & 0.876 & $0.804 \pm 0.005$ \\
\hline$\varepsilon_{5 / 2-}$ & 0.515 & 0.538 & 0.397 & 0.343 & $0.343 \pm 0.263$ \\
\hline$\xi_{5 / 2-}$ & 0.938 & 0.926 & 0.914 & 0.932 & $0.944 \pm 0.016$ \\
\hline$\eta_{5 / 2-}$ & -0.343 & -0.334 & -0.397 & -0.343 & $-0.321 \pm 0.018$ \\
\hline${ }^{4} D_{7 / 2}$ & -4.04 & -4.07 & -3.56 & -3.77 & $3.64 \pm 0.01$ \\
\hline${ }^{2} G_{7 / 2}$ & 0.108 & 0.107 & 0.088 & 0.099 & - \\
\hline${ }^{4} G_{7 / 2}$ & -0.215 & -0.214 & -0.180 & -0.199 & - \\
\hline$\varepsilon_{7 / 2+}$ & 0.357 & 0.355 & 0.417 & 0.712 & - \\
\hline$\xi_{7 / 2+}$ & 1.16 & 1.14 & 1.16 & 1.14 & 一 \\
\hline$\eta_{7 / 2+}$ & -0.462 & -0.459 & -0.426 & -0.422 & - \\
\hline
\end{tabular}

calculation, the ${ }^{4} P_{1 / 2}$ eigenphase shift is slightly larger than the PSA and the ${ }^{4} P_{3 / 2}$ eigenphase shift is smaller. For these eigenphase shifts, the difference from PSA is growing with higher energies, but the discrepancy of $A_{y}(\theta)$ at the maximum point between the theoretical prediction and the experimental data is decreasing, as will be seen in $\S 4.3$. This is because $A_{y}(\theta)$ is very sensitive to the ${ }^{4} P_{J}$ eigenphase shift only in the lower energy region, but not so much in the higher energy region [40]. We also find some deviation of the ${ }^{2} P_{1 / 2}$ eigenphase shifts from the results of PSA, as seen from the comparison with Fig. 12 of Ref. [39]. However, both ${ }^{2} P_{J}$ phase shifts have sizable and rapidly increasing imaginary components up to $10^{\circ}$ at $E_{N}=20 \mathrm{MeV}$. The ${ }^{2} P_{J^{-}}{ }^{4} P_{J}$ mixing parameters $\varepsilon_{J^{-}}$with $J=1 / 2$ and $3 / 2$ have large values with opposite signs to each other. In particular, $\varepsilon_{3 / 2^{-}}$is very large and reaches at 
$-45^{\circ}$ at $E_{N}=37-38 \mathrm{MeV}$. The eigenphase shifts of the $J^{\pi}=3 / 2^{-}$state are strongly influenced by the large ${ }^{2} P_{3 / 2}{ }^{4} P_{3 / 2}$ mixing for $E_{N} \geq 10 \mathrm{MeV}$, and the dominant components having the ${ }^{2} P_{3 / 2}$ and ${ }^{4} P_{3 / 2}$ quantum numbers are eventually interchanged beyond $\varepsilon_{3 / 2^{-}}>45^{\circ}$. Our result for $\varepsilon_{3 / 2}^{-}$, which is crucial for the correct description of $A_{y}(\theta)$ according to Refs. [38] and [41], agrees reasonably well with the PSA. Both of the ${ }^{2} P_{J}$ phase shifts with $J=1 / 2$ and $J=3 / 2$ have sizable and smoothly increasing imaginary components. For $D$ - and $F$-waves, ${ }^{4} D_{J}$ and ${ }^{2} F_{J}$ eigenphase shifts are repulsive and ${ }^{2} D_{J}$ and ${ }^{4} F_{J}$ are attractive. These are consistently reproduced with the predictions of the PSA, although ${ }^{2} F_{J}$ phase shifts of the PSA have large errorbars in the 4 to $10 \mathrm{MeV}$ region.

For the Coulomb effect in the $p d$ scattering, there exist several benchmark calculations $[42 ; 33 ; 43$; 44], using different approaches for the same realistic $N N$ forces like AV14 and AV18. For example, Ref. [42] compares the low-energy eigenphase shifts and mixing parameters for the $p d$ elastic scattering, predicted by the pair-correlated hyperspherical harmonics (PHH) approach and by the Faddeev-Noyes approach in the configuration space with appropriate Coulomb boundary conditions. Comparison with our results is not straightforward since the $N N$ force is different, but the difference of the phase-shift parameters between AV14 and AV18 is sometimes larger than that between fss2 and AV18+UR3N potentials. (Compare Table 1 with Table 1 of Ref. [42].) Another configuration approach in Refs. [43] and [44] essentially proves the consistency with the PHH approach. The benchmark calculation for the pd elastic-scattering observables by Deltuva et al. [33] shows the characteristic behavior of the Coulomb effect very similar to ours. (Compare Fig. 4 of Ref. [33] with our Figs. 5, 6 and 8, 9, 11, 12 below.) The extension of the configuration space approach in Ref. [44] to the $p d$ breakup differential cross sections shows that our Coulomb effect is qualitatively similar to theirs. (Compare Fig. 14 of Ref. [44] with our Fig. 13 below.)

\subsection{Effective-range parameters of the $n d$ scattering}

In this Section, we analyze the low-energy effective-range parameters and compare our results with the predictions by meson-exchange potentials. We will confirm the attractive feature of the fss 2 interaction in the doublet channel at very low-energies, which is already discussed to some extent in the preceding section. We first calculate the eigenphase shifts of the $n d$ scattering for the $J^{\pi}=1 / 2^{+}$and $J^{\pi}=3 / 2^{+}$ states. In these states, a coupled-channel theory of the effective-range parameters is in principle necessary, since the $S$ - and $D$-waves are coupled. Nevertheless, the single-channel effective-range expansion formula for the eigenphase shifts is reasonably applied to these states, since the $D$-wave eigenphase shifts and mixing parameters are very small compared to the $S$-wave ones for the energies below the deuteron breakup threshold. We however need high accuracy better than 0.01 degree for the eigenphase shifts, in order to extract effective-range parameters reliably. When the realistic deuteron wave function is used, it is very difficult in the momentum-space approach to maintain sufficient accuracy of the phase shifts at extremely low energies below $E_{\mathrm{cm}}=100 \mathrm{keV}$, since the solution of the AGS equation becomes very singular. We therefore increase the momentum discretization points from $n=6-6-5$ to $n$ $=6-10-5$, which corresponds to $3 \times 10+5=35$ points for $p$ for the $N N$ relative motion.

Table 2 The triton binding energy $E_{B}\left({ }^{3} \mathrm{H}\right), S$-wave scattering lengths ${ }^{2} a_{n d}$ and ${ }^{4} a_{n d}$, effective-range parameters $\left({ }^{4} r_{e}\right)_{n d}$ and $\left({ }^{2} \widetilde{r}_{e}\right)_{n d}$, predicted by fss2 for various model spaces with the maximum angular momentum of the $N N$ interaction $\left(I_{\max }\right)$ included. The nonlocal Gaussian potential with 15-point quadrature is used for fss 2 . In " $S+D$ ", only the ${ }^{3} S_{1}+{ }^{3} D_{1}$ and ${ }^{1} S_{0} N N$ channels are included. The pole energy for the doublet channel, $E_{Q}$, is also shown. The calculated deuteron binding energy is $2.2206 \mathrm{MeV}$. The momentum mesh points with $n=6-10-5$ are used.

\begin{tabular}{ccccccc}
\hline \hline$I_{\max }$ & $E_{B}\left({ }^{3} \mathrm{H}\right)(\mathrm{MeV})$ & ${ }^{2} a_{n d}(\mathrm{fm})$ & $\left({ }^{2} \widetilde{r}_{e}\right)_{n d}(\mathrm{fm})$ & $E_{Q}(\mathrm{keV})$ & ${ }^{4} a_{n d}(\mathrm{fm})$ & $\left({ }^{4} r_{e}\right)_{n d}(\mathrm{fm})$ \\
\hline$S+D$ & 8.247 & 0.65 & -149 & -147 & 6.30 & 1.84 \\
1 & 7.948 & 0.94 & -102 & -207 & 6.31 & 1.85 \\
2 & 8.213 & 0.72 & -133 & -163 & 6.30 & 1.84 \\
3 & 8.298 & 0.67 & -146 & -151 & 6.30 & 1.84 \\
4 & 8.307 & 0.66 & -148 & -148 & 6.30 & 1.84 \\
\hline
\end{tabular}




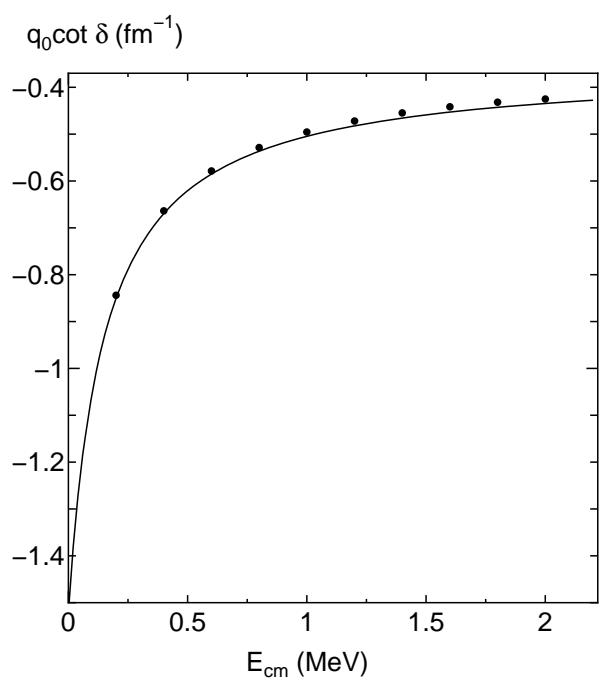

Fig. 2 The effective-range function $K\left(q_{0}\right)=q_{0} \cot \delta$ for the doublet $S$-state as a function of $E_{\mathrm{cm}}$. The filled circles are the fss 2 result and the solid curve shows the approximation by the effective-range parameters in Table 2 with $I_{\max }=4$.

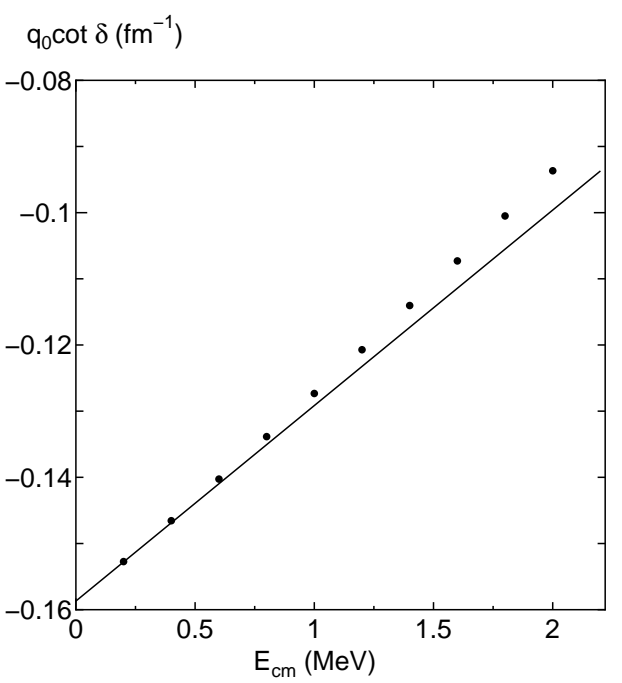

Fig. 3 Same as Fig. 2, but for $K\left(q_{0}\right)=q_{0} \cot \delta$ in the quartet $S$-state.

In the quartet $S$-channel, we expand the effective-range function which is the real part of the inverse scattering amplitude, $K\left(q_{0}\right)=q_{0} \cot \delta$, in a power series of $q_{0}^{2}$ :

$$
K\left(q_{0}\right)=-\frac{1}{a}+\frac{1}{2} r_{e} q_{0}^{2}+\mathcal{O}\left(q_{0}^{4}\right) .
$$

Here, $a$ is the scattering length, $r_{e}$ is the effective range, and $\delta=\delta_{0,3 / 2}^{3 / 2}$ is the quartet $S$-wave eigenphase shift. For the doublet channel, the effective-range function has a pole slightly below the elastic threshold $[45 ; 46 ; 47 ; 48]$. This is parametrized as

$$
K\left(q_{0}\right)=\frac{-\frac{1}{a}+\frac{1}{2} \widetilde{r_{e}} q_{0}^{2}+\mathcal{O}\left(q_{0}^{4}\right)}{1+\left(q_{0} / q_{Q}\right)^{2}}
$$

where the pole parameter $q_{Q}$ specifies the pole position and $\delta=\delta_{0,1 / 2}^{1 / 2}$ is the doublet $S$-wave eigenphase shift. It is reported in the early study using the $N / D$ formalism $[46 ; 47 ; 48]$ that the origin of this pole structure is brought about by the dominant single-nucleon exchange and other effects such as the twonucleon exchange. In the doublet channel, single-nucleon exchange, which is by far the longest-range force, is attractive and the nucleon in this channel can freely approach to the region where the other forces can act. On the other hand, the incident nucleon cannot penetrate deep inside the deuteron owing to the Pauli principle in the quartet channel. This is why the pole structure is found only in the doublet channel.

Table 2 lists the model-space dependence of the triton binding energy and effective-range parameters. The converged triton binding energy is $E_{B}\left({ }^{3} \mathrm{H}\right)=8.311 \mathrm{MeV}$ with $I_{\max }=6$. The difference by $15 \mathrm{keV}$ from the result in Ref. [3] $\left(E_{B}\left({ }^{3} \mathrm{H}\right)=8.326 \mathrm{MeV}\right)$ is supposed to be from the Gaussian representation of the interaction kernel. This can be interpreted in the following way. The Gaussian representation leads to the $4.4 \mathrm{keV}$ difference in the deuteron binding energy, which is the difference between $2.2250 \mathrm{MeV}$ (from the original result of fss2) [1] and $2.2206 \mathrm{MeV}$ (from the result of the nonlocal Gaussian potential for fss2). This difference is almost one-third of the $15 \mathrm{keV}$ difference in the triton binding energy in accordance with three $N N$ pairs in the triton.

The effective-range parameters in Table 2 with $I_{\max }=4$ are calculated from the sample points (filled circles) with energies between $E_{\mathrm{cm}}=200 \mathrm{keV}$ and $2 \mathrm{MeV}$, shown in Figs. 2 (the doublet channel) and 3 (the quartet channel), by applying the Schlessinger's point method [53] to the effective-range parameters. This method is convenient to take into account the contributions of higher-order terms in 
Table 3 Comparison of the $n d$ scattering lengths predicted by using fss2 $\left(I_{\max }=4\right)$ with other models. For the fss 2 results, the charge dependence of the $N N$ force is neglected. The heading $N N$ denotes the calculation using only the $N N$ force, and $N N+$ TM99 denotes the calculation including the Tucson-Melbourne 99 (TM99) $2 \pi$-exchange $3 N$ force $[49 ; 50]$. The results obtained by CD-Bonn 2000, AV18 and Nijm I are taken from Ref. [51] $\left(I_{\max }=5\right)$. The experimental values are taken from Ref. [52]. The values of ${ }^{4} a_{n d}$ are insensitive to the $3 N$ force.

\begin{tabular}{cccccc}
\hline \hline & \multicolumn{2}{c}{$E_{B}\left({ }^{3} \mathrm{H}\right)(\mathrm{MeV})$} & \multicolumn{2}{c}{${ }^{2} a_{n d}(\mathrm{fm})$} & ${ }^{4} a_{n d}(\mathrm{fm})$ \\
\hline Model & $N N$ & $N N+\mathrm{TM} 99$ & $N N$ & $N N+\mathrm{TM} 99$ & $N N(+\mathrm{TM} 99)$ \\
\hline fss2 & 8.307 & - & 0.66 & - & 6.30 \\
CD-Bonn 2000 & 8.005 & 8.482 & 0.925 & 0.569 & 6.347 \\
AV18 & 7.628 & 8.482 & 1.248 & 0.587 & 6.346 \\
Nijm I & 7.742 & 8.485 & 1.158 & 0.594 & 6.342 \\
\hline exp. & & 8.482 & \multicolumn{2}{c}{$0.65 \pm 0.04$} & $6.35 \pm 0.02$ \\
\hline
\end{tabular}

Eqs. (4.1) and (4.2) in a natural way and to approximate a function with a pole. The effective-range functions obtained by using the effective-range parameters with $I_{\max }=4$ in Table 2 are also shown in Figs. 2 and 3 by solid curves. This approximation up to $q_{0}{ }^{2}$ in Eqs. (4.1) and (4.2) is very good in the doublet channel, but the contribution of higher order terms than $q_{0}{ }^{2}$ is visible in the quartet channel. We reconfirm the pole structure in the doublet channel with $E_{Q}=-\left(3 \hbar^{2} q_{Q}^{2} / 4 M_{N}\right) \simeq-150$ $\mathrm{keV}$. From Table 2, we find that the spin-quartet scattering length ${ }^{4} a_{n d}$ has a large and positive value and is insensitive to the model space adopted. This insensitivity is related to the small distortion effect of the deuteron due to the Pauli repulsion of the nd interaction in this channel, which is a kinematical constraint imposed by the spin-isospin quantum numbers. On the other hand, the spindoublet scattering length ${ }^{2} a_{n d}$ is subject to a strong channel coupling effect. The prediction from the $S+D$ calculation, including the ${ }^{3} S_{1}+{ }^{3} D_{1}$ and ${ }^{1} S_{0} N N$ channels only, is close to the converged result. However, $P$-wave $N N$ interaction gives a repulsive effect and a well-converged value is achieved after many partial waves, up to at least $G$-wave of the $N N$ interaction, are included. The values of $\left|E_{Q}\right| /\left({ }^{2} a_{n d}\right) \sim 220 \mathrm{keV} / \mathrm{fm}$ are almost independent of the model space. This linear correlation has already been suggested in Ref. [48] for separable potentials. A strong correlation between the triton binding energy and ${ }^{2} a_{n d}$, which is known as the Phillips line [54], is also apparent. The correlation between the triton binding energy and the doublet scattering length ${ }^{2} a_{n d}$ can be explained in the following way. When the incident neutron energy approaches to zero, the phase shift becomes close to zero more slowly if $n d$ interaction is more attractive. In this situation, the effective-range function $K\left(q_{0}\right) \sim k / \delta \sim-1 /\left({ }^{2} a_{n d}\right)$ is larger in absolute value, which corresponds to a smaller ${ }^{2} a_{n d}$.

In Table 3, we compare the triton binding energy and ${ }^{2} a_{n d}$ with some results of other extensive calculations using meson-exchange potentials and the $3 N$ forces [51]. We should note that the charge dependence is included in the calculations of Ref. [51], but not included in our fss 2 predictions. The charge dependence affects ${ }^{2} a_{n d}$ appreciably, as well as the triton binding energy. We can estimate this effect from the slope of the Phillips line $-0.686 \mathrm{fm} / \mathrm{MeV}$ for fss 2 and the charge dependence effect of the triton binding energy of about $200 \mathrm{keV}$. From these values, the charge dependence correction is estimated to be about $0.10-0.14 \mathrm{fm}$ and we obtain ${ }^{2} a_{n d}$ for fss 2 to be $0.76-0.80 \mathrm{fm}$. Table 3 shows that ${ }^{2} a_{n d}$ is more than $0.9 \mathrm{fm}$ when only $N N$ meson-exchange potentials are used. The experimental values of ${ }^{2} a_{n d}$ and triton binding energy are reproduced only when the $3 N$ force is included. The $3 N$ effect on the doublet scattering length ${ }^{2} a_{n d}$ is more than $0.4 \mathrm{fm}$. As to the doublet scattering length ${ }^{2} a_{n d}$, the $3 N$ force is more influential than the charge dependence of the $N N$ force. The model fss 2 almost reproduces the experimental values of the triton binding energy and ${ }^{2} a_{n d}$ simultaneously without the $3 N$ force.

\subsection{Total cross sections}

In this Section, we mainly show the results for the nd total cross sections and discuss the energy dependence of the $S$-wave contribution using the effective-range expansion Eqs. (4.1) and (4.2). The "S-wave" means that the orbital angular momentum between the neutron and the deuteron is zero. The elastic and breakup total cross sections for $E_{n} \leq 40 \mathrm{MeV}$, predicted using fss2, are plotted in Fig. 4, 
together with the experimental data. Although some discrepancies might exist around $E_{n} \sim 10 \mathrm{MeV}$, the elastic and breakup total cross sections calculated from the optical theorem are well reproduced. In Table 4, we show the $S$-wave contributions from the quartet $\left({ }^{4} S\right)$ and doublet $\left({ }^{2} S\right)$ channels to the total cross sections, calculated from the effective-range parameters in Table 2 . We find that the total cross sections are dominated by the $S$-wave contribution, which is about $60 \%$ for $E_{n}=3 \mathrm{MeV}$. The $S$ wave contribution is not close to $100 \%$, since the $P$-wave and $D$-wave contribution is appreciable even in the energies below the deuteron breakup threshold. We should note that the $D$-wave component of the deuteron wave function can couple with the $n d D$-wave components for the relative motion. The quartet state is far more important than the doublet state owing to the small values of $\left|q_{0} \cos \delta\right|$, even considering the statistical factor $\left(2 S_{c}+1\right)$. As the energy increases, the contributions from the doublet states become appreciable avoiding the effect of the pole structure just below the elastic threshold, but is still less than $8 \%$ at $E_{n}=3 \mathrm{MeV}$. This implies a very special situation in which the extra attraction to the ${ }^{2} S_{1 / 2}$ state by the $3 N$ force is unimportant in reproducing the elastic differential cross sections of the low-energy $n d$ scattering. The low-energy $n d$ cross sections are mainly determined by the magnitude of the strongly repulsive ${ }^{4} S_{3 / 2}$ eigenphase shift.

When the cutoff Coulomb force is introduced to the calculation, the elastic and total cross sections diverge as the cutoff radius $\rho$ is increased. However, the total breakup cross sections derived from the unitary condition remain rather stable for the change of $\rho$. For example, the $n d$ breakup total cross section $48.09 \mathrm{mb}$ at $E_{n}=5 \mathrm{MeV}$ is reduced to $33.78 \mathrm{mb}(33.74 \mathrm{mb}, 32.29 \mathrm{mb})$ if $\rho=8 \mathrm{fm}(16 \mathrm{fm}, 20$ $\mathrm{fm})$ is used. These are compared with the experimental values $\sigma_{n d}(b r)=(34 \pm 6) \mathrm{mb}$ at $E_{n}=4.9 \mathrm{MeV}$ [58] and $\sigma_{p d}(b r)=(21.5 \pm 1.4) \mathrm{mb}$ [60], cited in Ref. [61]. Our calculation somewhat overestimates the experimental values, but the empirical Coulomb reduction factor of $\left(65 \pm{ }_{13}^{19}\right) \%$ is quite close to our values $67 \%-70 \%$. This reduction factor monotonically increases to unity as $\rho$ is increased; $85 \%$ at $E_{p}=8 \mathrm{MeV}, 90 \%$ at $10 \mathrm{MeV}$, and almost unity beyond $30 \mathrm{MeV}$. The $p d$ total reaction cross sections at $23-46 \mathrm{MeV}$ measured by the direct method [62] show almost no difference between $\sigma_{n d}(b r)$ and $\sigma_{p d}(b r)$ within the errorbars, which is in accordance with our predictions.

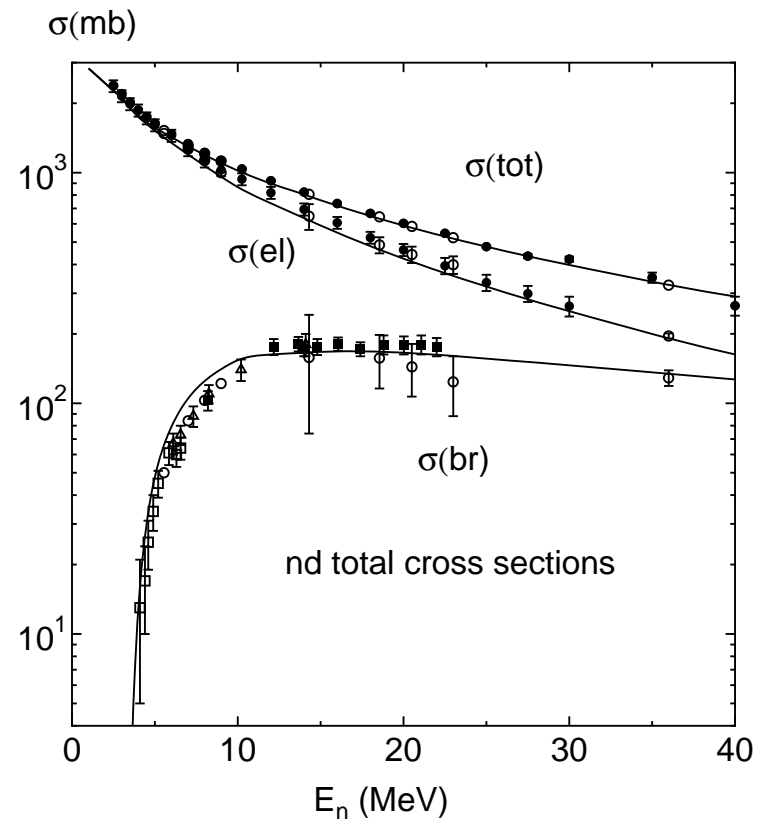

Fig. 4 The $n d$ elastic and breakup total cross sections up to $E_{n}=40 \mathrm{MeV}$, compared with the experimental data. The experimental data are taken from Refs. [55] for filled circles, [56] for open circles, [57] for filled squares, [58] for open squares, and [59] for filled triangles. 
Table 4 The quartet $\left({ }^{4} S\right)$ and doublet $\left({ }^{2} S\right) S$-wave contributions to the low-energy $n d$ total cross sections $\sigma_{\text {tot }}$, calculated from the effective-range parameters for fss 2 with $I_{\max }=4$ in Table 2 .

\begin{tabular}{ccccc}
\hline \hline$E_{n}$ & $0 \mathrm{MeV}$ & $1 \mathrm{MeV}$ & $2 \mathrm{MeV}$ & $3 \mathrm{MeV}$ \\
\hline${ }^{4} S(\mathrm{mb})$ & $3325(99.5 \%)$ & $2055(73 \%)$ & $1467(59 \%)$ & $1128(54 \%)$ \\
${ }^{2} S(\mathrm{mb})$ & $18.2(0.5 \%)$ & $122.4(4.3 \%)$ & $158.4(6.3 \%)$ & $165.5(7.9 \%)$ \\
$S$-wave $(\mathrm{mb})$ & 3343 & $2177(77 \%)$ & $1625(66 \%)$ & $1294(62 \%)$ \\
$\sigma_{\text {tot }}(\mathrm{mb})$ & - & 2832 & 2480 & 2104 \\
\hline exp. $(\mathrm{mb})$ & - & $2893.6 \pm 18.2[63]$ & $2550.6 \pm 11.1[63]$ & $2158.0 \pm 7.2[63]$ \\
& & $2854 \pm 39[64]$ & $2537 \pm 10[65]$ & $2240 \pm 90[66]$ \\
& & $3110 \pm 200[66]$ & $2600 \pm 80[66]$ & $2160 \pm 86[67]$ \\
\hline
\end{tabular}

\subsection{Elastic differential cross sections}

We show in Figs. $5-6$ the $n d$ and $p d$ differential cross sections predicted by fss 2 for incident energies from $E_{N}=1$ to $95 \mathrm{MeV}$. For each energy, two curves are plotted corresponding to calculations for $n d$ scattering (dashed curves) and $p d$ scattering (solid curves). The $n d$ data shown with bars should be compared with the dashed curves and the $p d$ data with circles and others correspond to the solid curves. Some experimental data are obtained at slightly different energies from our calculation. We find that the experimental data are well reproduced including the nuclear-Coulomb interference region. In particular, the agreement at $E_{N} \leq 22.7 \mathrm{MeV}$ is very well, which is a common feature with the predictions by the meson-exchange potentials including the $3 N$ force $[21 ; 68 ; 30 ; 31]$.

In general, the Coulomb effect for the elastic scattering is very large at low energies and gradually reduces as the energy increases. At $E_{N}=1 \mathrm{MeV}$, the $n d$ and $p d$ differential cross sections are quite different from each other. In the low-energy region with $E_{N}<10 \mathrm{MeV}$, Coulomb effect for differential cross sections is mainly observed at forward and backward angles, while in the higher-energy region it is confined only to the forward angles with $\theta_{\mathrm{cm}} \leq 30^{\circ}$. In order to discuss the angular distribution of the differential cross sections below the deuteron breakup threshold, we took the $J$-average of the eigenphase shifts in Ref. [23] with respect to the definite $S_{c}$ and orbital angular momentum $\ell$. We further adopted a simple approximation proposed by Doleschall et al. [69], which is called the "Coulomb externally corrected approximation" in Ref. [31]. In this approximation, we simply add the Coulomb amplitude to the $n d$ scattering amplitude with the Coulomb phase factors. This prescription yields a large overestimation of the differential cross sections [23; 70], especially for low energies. The almost complete reproduction of the differential cross sections is obtained by taking into account the difference between the $n d$ and $p d$ nuclear phase shifts of low-partial waves [23]. This implies that the Coulomb distortion effect to the nuclear interaction is very important in the low-energy region.

We compare in Table 5 quantitatively the $p d$ differential cross sections at the minimum points around $\theta_{\mathrm{cm}}=90^{\circ}-130^{\circ}$ (diffraction minima) between the model fss 2 and the $p d$ experimental data, in order to investigate the energy dependence. The $n d$ differential cross sections calculated by fss2 are also shown to examine the Coulomb effect. We should note that, in energies above the deuteron breakup threshold, many partial waves contribute and yet the shape of the differential cross sections is rather simple owing to the strong cancellation. Therefore, the energy dependence at the diffraction minima affords a very crucial test of the $N N$ interaction. This energy dependence is very important to discuss the effect of the $3 N$ force for the meson-exchange potentials. The disagreement at the diffraction minima between theoretical predictions and experimental data is generally known as the Sagara discrepancy [75]. We find that, on the low-energy side $E_{p} \leq 18 \mathrm{MeV}$, the minimum values of the $p d$ differential cross sections calculated by fss2 reproduce the experimental data with an inaccuracy of less than $2 \%$. There is no strong energy dependence reported in Ref. [75], and our discrepancies are much smaller than the $6-9 \%$ overestimation predicted in Ref. [30]. (See Fig. 8 of Ref. [30].) The $p d$ calculation of Ref. [68], using the AV18 potential, still gives the $4-7 \%$ overestimation of the cross section minima at $E_{p}<18 \mathrm{MeV}$ because of the Coulomb effect. (See Table II of Ref. [68].) This overestimation, however, is largely cancelled by the introduction of the Urbana URIX $3 N$ force, resulting in a good agreement with the experimental data. This fact is also confirmed by Ishikawa. (See Figs. 3 and 4 of [43].) The discrepancy from the experimental data is less than $3 \%$ in this energy region, which is a comparable accuracy to our results. Here we again find that in fss 2 the attractive 

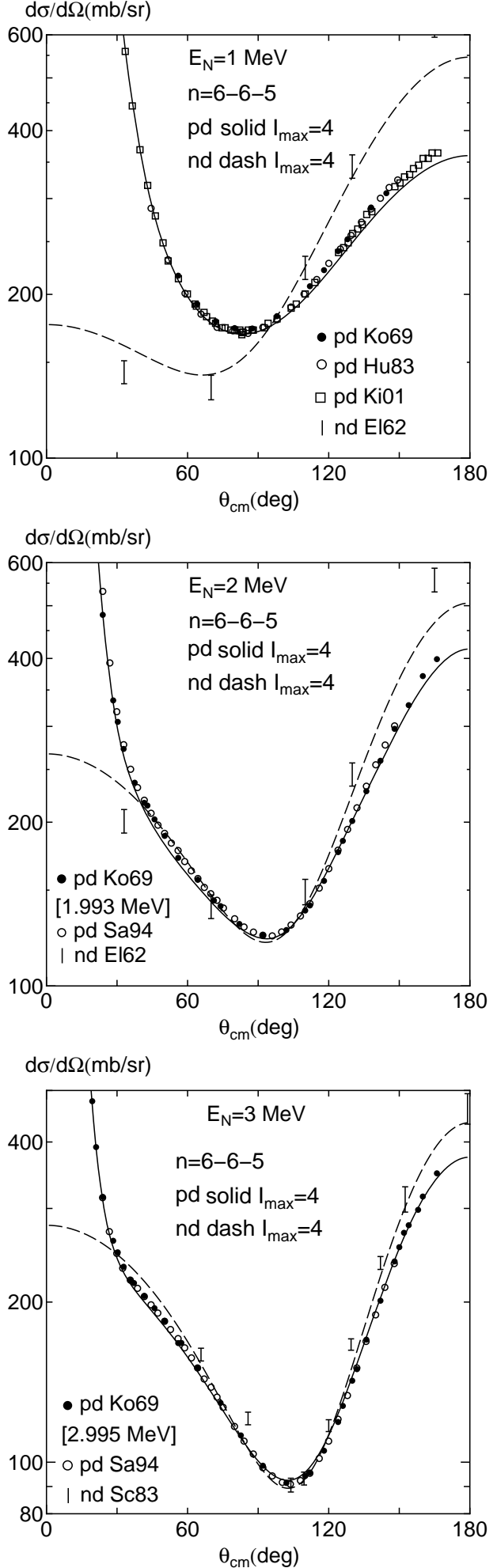

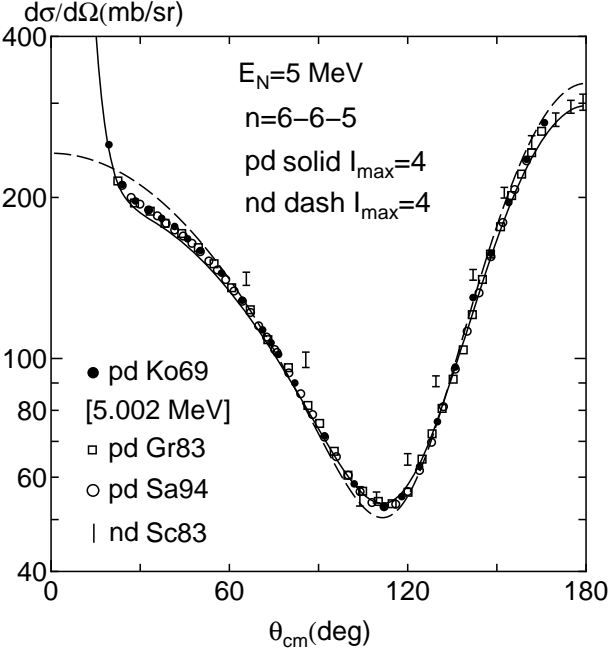

$\mathrm{d} \sigma / \mathrm{d} \Omega(\mathrm{mb} / \mathrm{sr})$

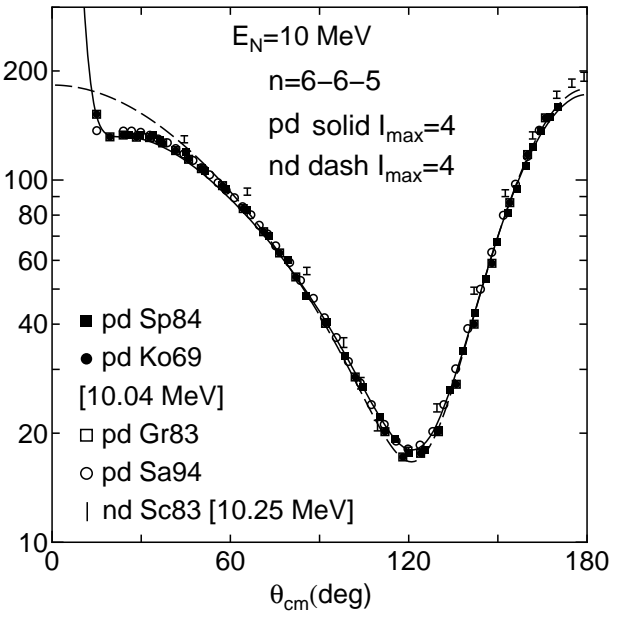

$\mathrm{d} \sigma / \mathrm{d} \Omega(\mathrm{mb} / \mathrm{sr})$

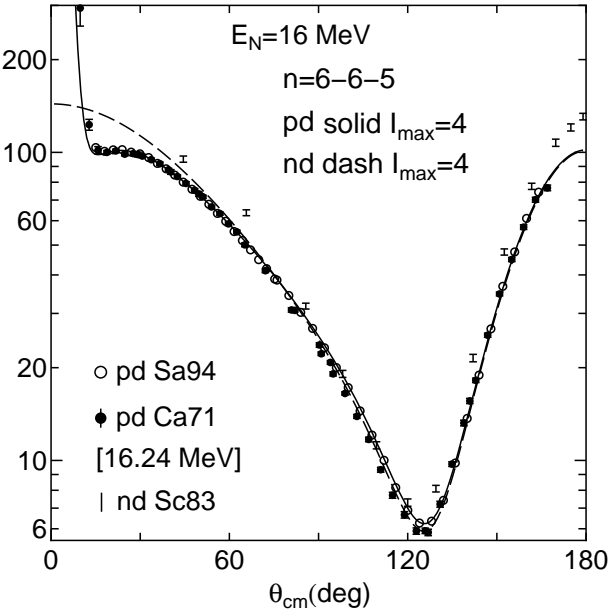

Fig. $5 n d$ and $p d$ differential cross sections from $E_{N}=1$ to $16 \mathrm{MeV}$, compared with the experimental data. The solid curve shows the $p d$ prediction by fss 2 with the screened Coulomb force and the dashed curve shows the $n d$ calculation. The experimental data are taken from Refs. [71] for Ko69, [72] for Hu83, [73] for Ki01, [74] for El62, [75] for Sa94, [55] for Sc83, [76] for Gr83, [77] for Sp84, and Ref. [78] for Ca71. 

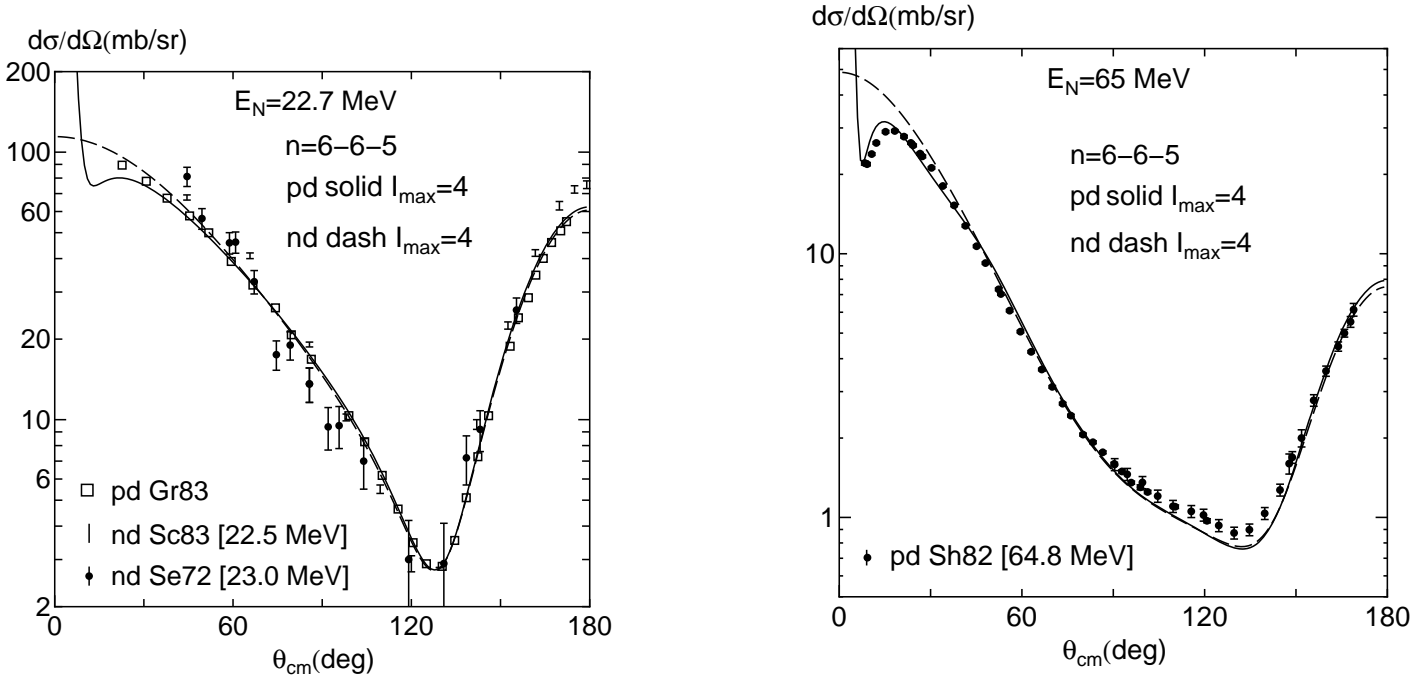

$\mathrm{d} \sigma / \mathrm{d} \Omega(\mathrm{mb} / \mathrm{sr})$
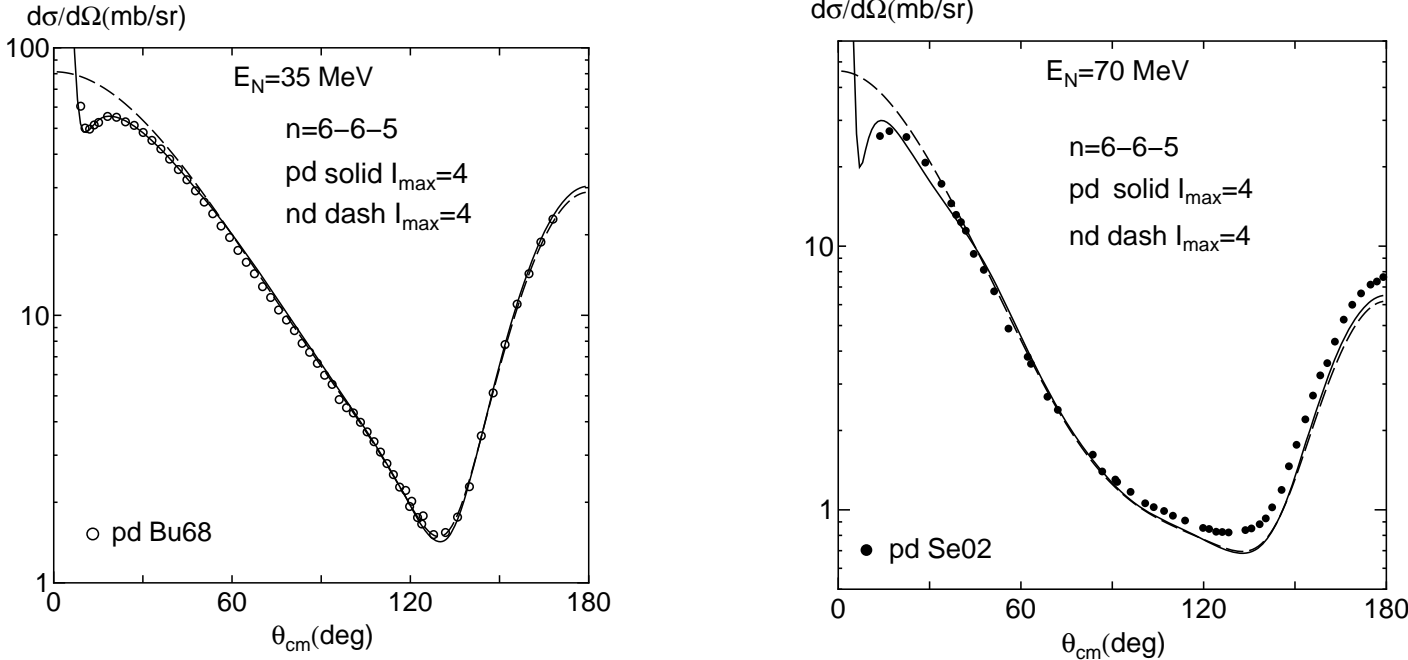

$\mathrm{d} \sigma / \mathrm{d} \Omega(\mathrm{mb} / \mathrm{sr})$
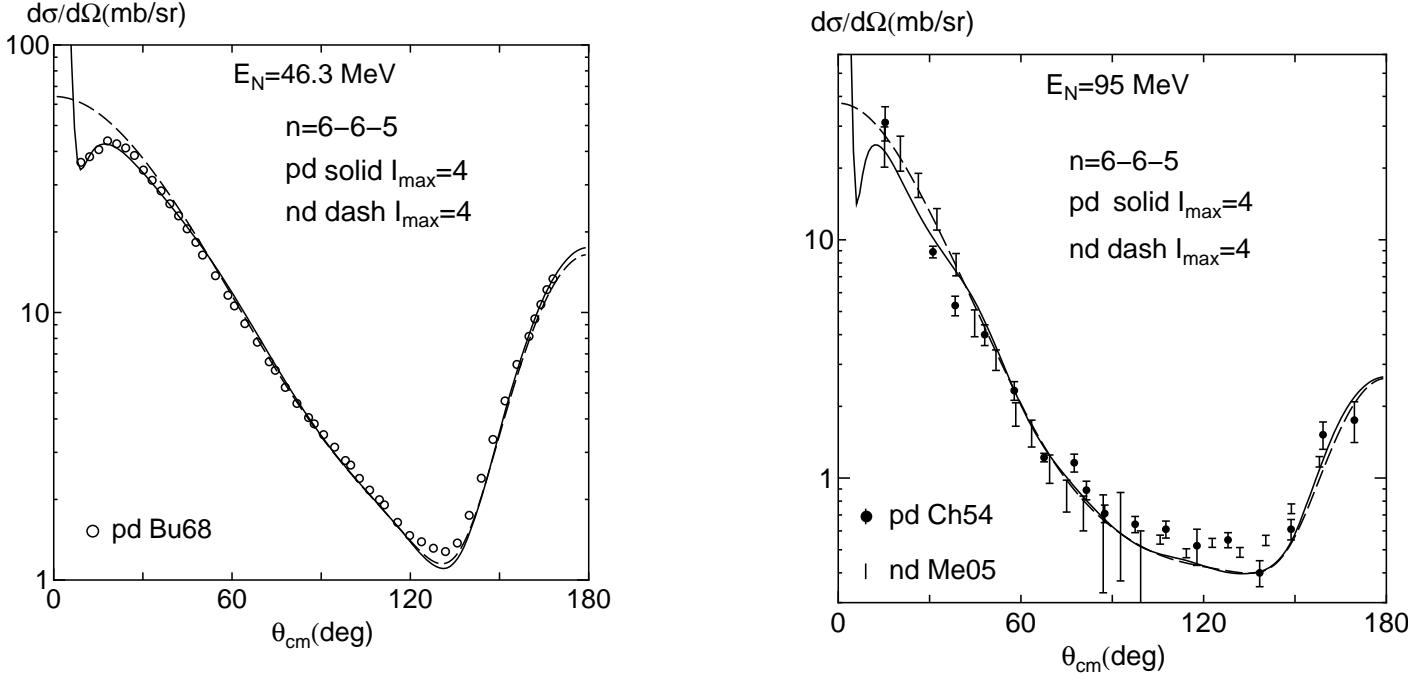

Fig. $6 n d$ and $p d$ differential cross sections from $E_{N}=22.7$ to $95 \mathrm{MeV}$, compared with the experimental data. The experimental data are taken from Refs. [56] for Se72, [79] for Bu68, [80] for Sh82, [81] for Se02, [82] for Ch54, and Ref. [83] for Me05. The others are same as in Fig. 5. 
effect by the $3 N$ force is already taken into account at the two-nucleon interaction level, and the nice agreement with the experimental data is achieved without the three-body force. It should be stressed that this accords with the good reproduction of the triton binding energy [3] and $n d$ doublet scattering length ${ }^{2} a_{n d}$ by fss 2 , as already discussed in the preceding subsections. Concerning the Coulomb effect, it is rather small at the diffraction minima in the low-energy region so long as $E_{p} \geq 2 \mathrm{MeV}$. This is very similar to the result in Ref. [68] for $E_{p}<18 \mathrm{MeV}$. (Compare Table 5 and Table II of Ref. [68].) As the energy increases, the fss 2 results tend to go below the data at the minimum point and the deficiency amounts to $14 \%$ at $E_{p}=28 \mathrm{MeV}$ and $16 \%$ at $E_{p}=70 \mathrm{MeV}$. The discrepancy at $E_{p} \sim$ $70 \mathrm{MeV}$ is similar to the predictions by the meson-exchange potentials in Refs. [21; 81]. In the higher energy region with $E_{p} \geq 65 \mathrm{MeV}$, it is reported that the Coulomb effect to the diffraction minima is rather small and the explicit inclusion of the $\Delta$ isobar degree of freedom becomes more important to decrease the discrepancies (namely to increase the differential cross sections) [31; 85]. In our case, the Coulomb effect works in the direction to reduce the differential cross sections at the diffraction minima beyond $E_{p} \sim 28 \mathrm{MeV}$. This behavior might be due to inadequate treatments such as the too-small Coulomb cutoff parameter or/and the insufficient partial waves.

\subsection{Analyzing powers}

The vector analyzing powers of the nucleon $A_{y}(\theta)$, predicted by the model fss 2 , are compared with a large amount of the experimental data in Figs. $8-9$ for the energies from $E_{N}=1$ to $65 \mathrm{MeV}$. Almost all the polarization data are for the $p d$ or $d p$ scattering and the accurate comparison with the experimental data requires the inclusion of the Coulomb force. The nd data shown with bars should be compared with the dashed curves and the $p d$ data with circles and others correspond to the solid curves. We should note that the polarization observables are more sensitive to the truncation of the model space and to the Coulomb effect than the differential cross sections. They are also affected by

Table 5 Comparison of the minimum values of the elastic differential cross sections, predicted by fss 2 , with the experimental data. The minimum value $(d \sigma / d \Omega)_{\min }(N d)$ at the minimum point $\theta_{\mathrm{cm}}$ is derived for the $n d$ or $p d$ scattering at the nucleon incident energy $E_{N}=E_{n}$ or $E_{p}$

\begin{tabular}{ccccccc}
\hline $\begin{array}{c}E_{N} \\
(\mathrm{MeV})\end{array}$ & $\begin{array}{c}\theta_{\mathrm{cm}} \\
(\mathrm{deg})\end{array}$ & $\begin{array}{c}(d \sigma / d \Omega)_{\min }(n d) \\
(\mathrm{mb})\end{array}$ & $\begin{array}{c}\theta_{\mathrm{cm}} \\
(\mathrm{deg})\end{array}$ & $\begin{array}{c}(d \sigma / d \Omega)_{\min }(p d) \\
(\mathrm{mb})\end{array}$ & $\begin{array}{c}(d \sigma / d \Omega)_{\min }^{\exp }(p d) \\
(\mathrm{mb})\end{array}$ & Ref. \\
\hline 1 & 66 & 142.1 & 83 & 169.0 & $170.2 \pm 1.3$ & {$[73]$} \\
2 & 93 & 120.3 & 93 & 122.1 & $123.7 \pm 1.0$ & {$[75]$} \\
2.5 & 99 & 103.8 & 99 & 106.5 & $104.5 \pm 0.8$ & {$[75]$} \\
3 & 103 & 89.3 & 103 & 92.5 & $91.1 \pm 0.7$ & {$[75]$} \\
4 & 108 & 65.4 & 107 & 69.2 & $69.8 \pm 0.5$ & {$[75]$} \\
5 & 112 & 50.4 & 112 & 53.6 & $52.7 \pm 0.4$ & {$[75]$} \\
6 & 114 & 39.6 & 114 & 42.3 & $41.8 \pm 0.3$ & {$[75]$} \\
6.5 & 115 & 35.3 & 116 & 37.7 & $37.2 \pm 0.3$ & {$[75]$} \\
7 & 116 & 31.5 & 117 & 33.7 & $32.9 \pm 0.2$ & {$[75]$} \\
8 & 118 & 25.2 & 119 & 27.1 & $26.7 \pm 0.2$ & {$[75]$} \\
8.5 & 119 & 22.7 & 120 & 24.4 & $24.3 \pm 0.2$ & {$[75]$} \\
9 & 120 & 20.4 & 120 & 22.0 & $21.8 \pm 0.2$ & {$[75]$} \\
10 & 121 & 16.7 & 121 & 18.0 & $18.0 \pm 0.2$ & {$[75]$} \\
12 & 123 & 11.4 & 123 & 12.3 & $12.2 \pm 0.1$ & {$[75]$} \\
14 & 124 & 8.05 & 125 & 8.61 & $8.7 \pm 0.1$ & {$[75]$} \\
16 & 125 & 5.89 & 126 & 6.23 & $6.2 \pm 0.1$ & {$[75]$} \\
18 & 126 & 4.48 & 126 & 4.69 & $4.7 \pm 0.1$ & {$[75]$} \\
22.7 & 128 & 2.72 & 128 & 2.75 & $2.89 \pm 0.03$ & {$[76]$} \\
28 & 129 & 1.92 & 129 & 1.88 & $2.19 \pm 0.02$ & {$[84]$} \\
35 & 129 & 1.49 & 130 & 1.42 & $1.52 \pm 0.04$ & {$[79]$} \\
46.3 & 131 & 1.15 & 130 & 1.10 & $1.28 \pm 0.02$ & {$[79]$} \\
65 & 131 & 0.775 & 132 & 0.759 & $0.873 \pm 0.045$ & {$[80](64.8 \mathrm{MeV})$} \\
70 & 133 & 0.695 & 133 & 0.684 & $0.812 \pm 0.004$ & {$[81]$} \\
\hline
\end{tabular}


the slight change of the nuclear phase shifts. The enhancement of $A_{y}(\theta)$ in the forward angular region is qualitatively reproduced by introducing the screened Coulomb force, but not quantitatively. In the low energy region $E_{p} \leq 7 \mathrm{MeV}$ the enhancement in $\theta_{\mathrm{cm}} \leq 60^{\circ}$ is not sufficient in the present calculations. In the energy region $E_{N} \leq 14 \mathrm{MeV}$, the Coulomb effect is appreciable at the maximum points around $\theta_{\mathrm{cm}} \sim 120^{\circ}$ and make the agreement with the experimental data worse. In the energy region $E_{N}=16$ - $30 \mathrm{MeV}$, the shape of $A_{y}(\theta)$ changes and a clear minimum point appears. At the minimum points, the Coulomb effect can be observed. In the higher energy region, the Coulomb effect diminishes and is confined to the forward angular region. In the energy region $E_{p}=30-65 \mathrm{MeV}$, there is no large discrepancy in $A_{y}(\theta)$ between the theory and experiment.

The long-standing $A_{y}$ puzzle for the large discrepancy between the theory and experiment in the low-energy region $E_{N} \leq 20 \mathrm{MeV}$ still persists even in our calculations. In order to examine the discrepancy more quantitatively, we calculate the relative magnitude at the maximum point according to

$$
\mathrm{RA}_{\max }=\frac{A_{y}^{\mathrm{th}}\left(\theta_{\max }\right)}{A_{y}^{\exp }\left(\theta_{\max }\right)}
$$

where $A_{y}^{\mathrm{th}}\left(\theta_{\max }\right)$ and $A_{y}^{\exp }\left(\theta_{\max }\right)$ are the maximum values of $A_{y}(\theta)$ in the theoretical predictions and the experimental data, respectively. In Fig. 7, we show RAmax's in the energy region $E_{N} \leq 19 \mathrm{MeV}$, and compare them with the AV18 predictions in Ref. [102]. From this figure, the deficiency of the $A_{y}(\theta)$ at the maximum point is about $15-20 \%$ in our case, which is smaller than $25-30 \%$ in the predictions by AV18. In our calculations, the $A_{y}$ puzzle for the $p d$ scattering is more serious than for the $n d$ scattering. However, in the $p d$ scattering, this discrepancy diminishes as the energy increases. The realistic $N N$ interaction AV18 does not reproduce $A_{y}(\theta)$ unless the ${ }^{3} P_{J} N N$ interactions is modified

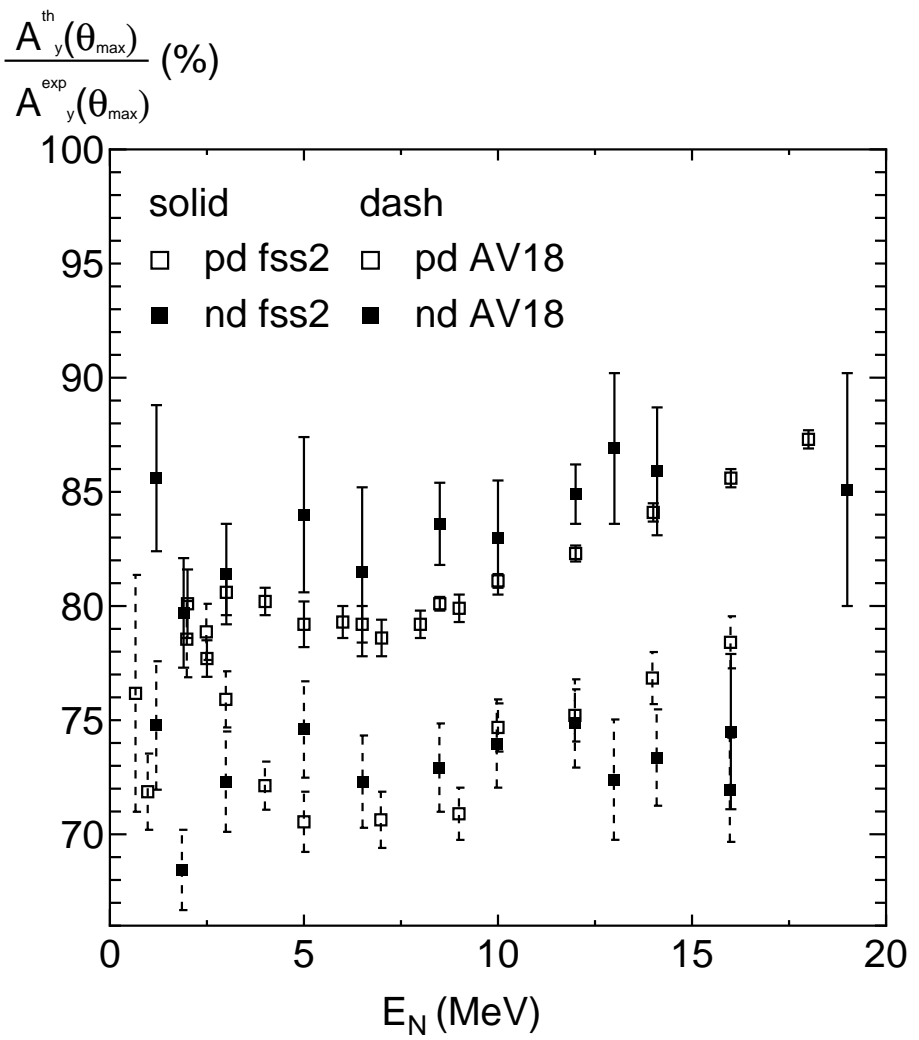

Fig. 7 Relative magnitudes of the theoretical predictions to the experimental data for the $p d$ (open squares) and $n d$ (filled squares) analyzing powers in the maximum angular points of $A_{y}(\theta)$. See Eq. (4.3). The experimental data are taken from Refs. $[75 ; 86]$ for the $p d$ scattering, and Refs. $[87 ; 88 ; 89 ; 90 ; 91 ; 92 ; 93]$ for the $n d$ scattering. The results for AV18 are also shown by open and filled squares with dashed errorbars for comparison. The nd calculations using AV18 are from Witala [94] and pd calculations from Kievsky [95]. 

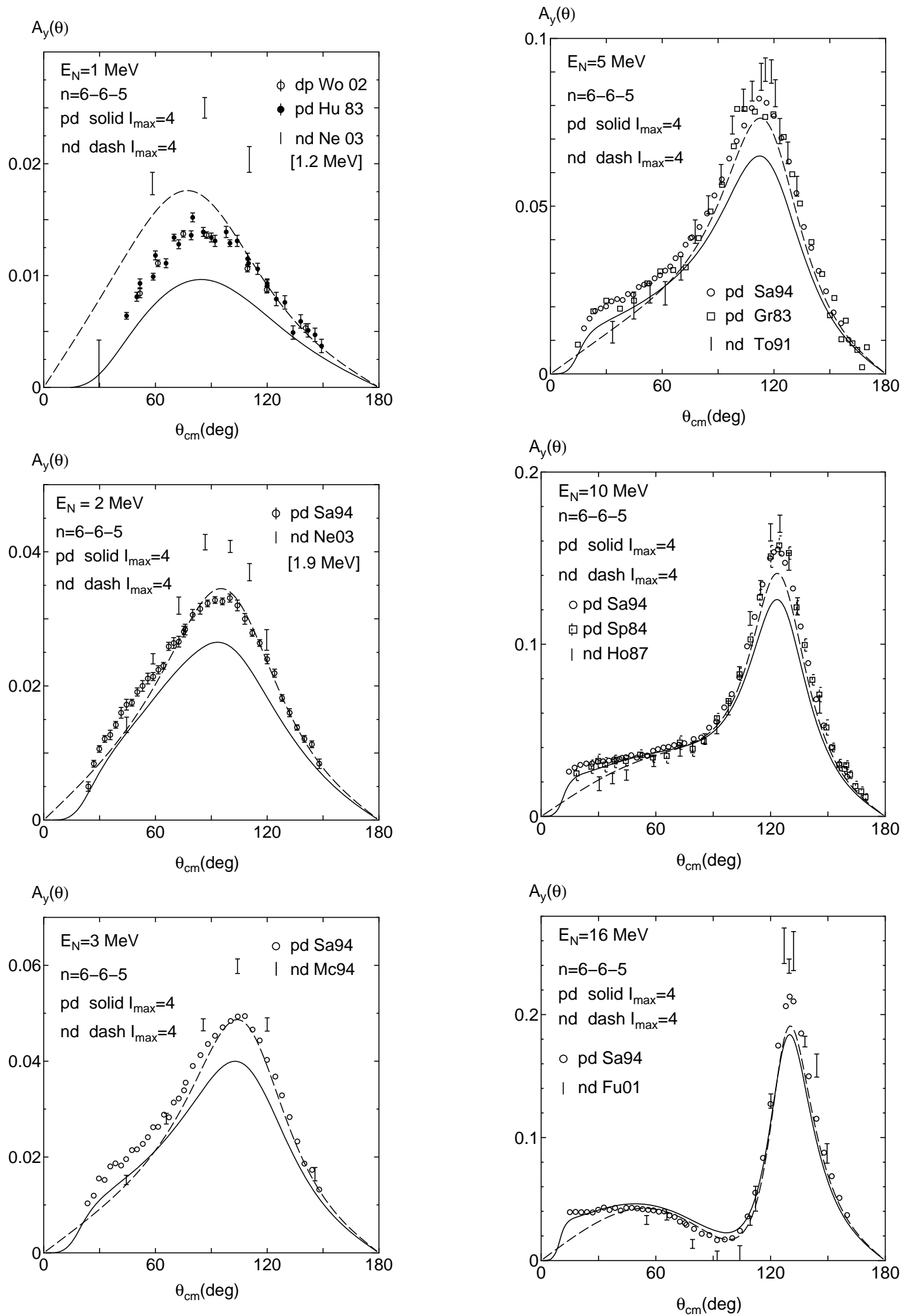

Fig. 8 Nucleon analyzing power $A_{y}(\theta)$ for the $n d$ and $p d$ elastic scattering from $E_{N}=1$ to $16 \mathrm{MeV}$, compared with the experimental data. The experimental data are taken from Refs. [96] for Wo02, [87] for Ne03, [88] for Mc94, [89] for To91, [90] for Ho87, and Ref. [92] for Fu01. The others are same as in Fig. 5. 

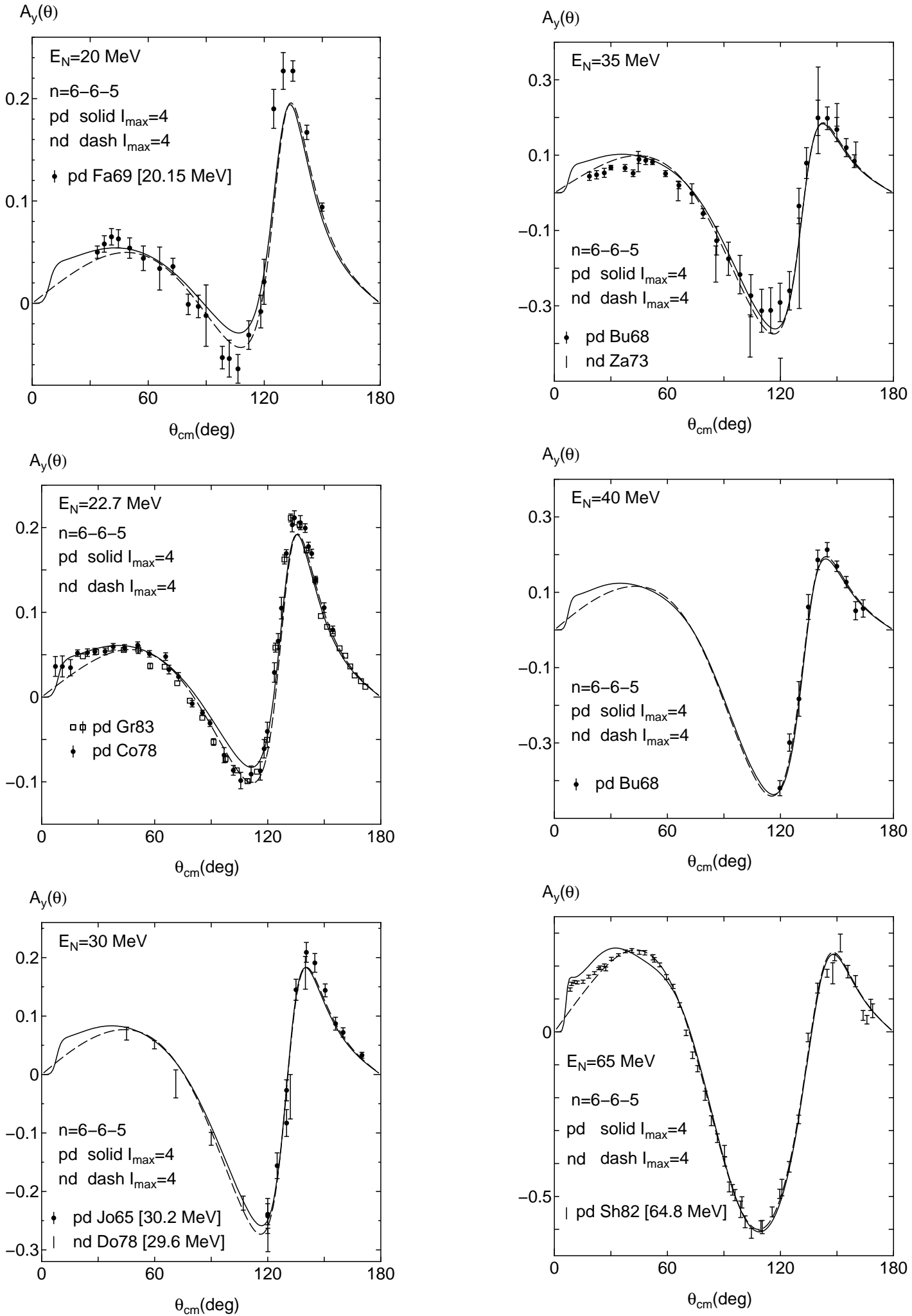

Fig. 9 Nucleon analyzing power $A_{y}(\theta)$ for the $n d$ and $p d$ elastic scattering from $E_{N}=20$ to $65 \mathrm{MeV}$, compared with the experimental data. The experimental data are taken from Refs. [97] for Fa69, [98] for Co78, [99] for Jo65, [100] for Do78, [79] for Bu68, [101] for Za73, and Ref. [80] for Sh82. The others are same as in Fig. 8. 
unrealistically [41]. The $N N$ interaction of the model fss 2 reproduces the empirical ${ }^{3} P_{J}$ phase shifts within an accuracy of one degree at energies less than $300 \mathrm{MeV}$ [1]. In Ref. [41], the authors claim that the ${ }^{4} P_{1 / 2}$ and $\varepsilon_{3 / 2}^{-}$should be appreciably modified by the $3 N$ force, in order to improve $A_{y}(\theta)$. In Refs. [103; 44], a phenomenological $3 N$ force including the tensor and spin-orbit components is incorporated to reproduce the experimental data for the low-energy $A_{y}(\theta)$ and $T_{21}(\theta)$. The energy dependence of these observables is nicely reproduced by the $3 N$ spin-orbit and tensor forces with moderate strength. It is interesting to figure out if these non-central $3 N$ forces are consistent with predictions by the effective field theory.

In some old predictions using the realistic separable potentials $[69 ; 104 ; 105 ; 106], A_{y}(\theta)$ 's at some energies are very well reproduced. Our QM $N N$ interactions and the separable potentials, which are characterized by the strong nonlocality in the configuration space, are both considered to have quite differential off-shell properties from the meson-exchange potentials. It is expected that the nonlocal description of the short-range repulsion is related to our improvement in $A_{y}$ puzzle, although it is difficult to pin down some particular eigenphase shifts.

We show the vector analyzing powers of the deuteron $i T_{11}(\theta)$ in Fig. 10, and compare them with the experimental data. Since all these observables are measured from the $d p$ elastic scattering, the inclusion of the Coulomb force is necessary as for $A_{y}(\theta)$. We find a problem that in low energies the peak heights of $i T_{11}(\theta)$ are appreciably lowered by the effect of the Coulomb force, which is already pointed out in Ref. [38]. This situation is similar to $A_{y}(\theta)$ and is not surprising, since $A_{y}(\theta)$ and $i T_{11}(\theta)$ have rather similar spin structure. The peak height around $\theta_{\mathrm{cm}} \sim 120^{\circ}$ is too low by $10-15 \%$ in the energy region $E_{d} \leq 28 \mathrm{MeV}$. The introduction of the Coulomb force leads to the slight enhancement at $\theta_{\mathrm{cm}} \leq 60^{\circ}$. The experimental data in $E_{d} \leq 14 \mathrm{MeV}$ are very well reproduced in this angular region. However, our calculations do not reproduce the behavior of $A_{y}(\theta)$ and $i T_{11}(\theta)$ in the forward angles simultaneously with a common cutoff radius $\rho$, which is also seen in other calculations $[30 ; 31 ; 68 ; 70]$. In the higher energy region, the experimental data are well reproduced, although error bars are rather large.

In Figs. 11 - 12, the deuteron tensor analyzing powers $T_{20}(\theta), T_{21}(\theta)$ and $T_{22}(\theta)$ in $E_{d} \leq 130$ $\mathrm{MeV}$ are compared with the $d p$ experimental data. Our results using fss 2 show comparatively better agreement with the experimental data than the vector analyzing powers. In the higher energy region with $E_{d}>56 \mathrm{MeV}$, experimental data with smaller error bars are desirable. The tensor analyzing powers in the low energy region are largely influenced by the Coulomb effect. In particular, the shape of $T_{20}(\theta)$ and $T_{21}(\theta)$ at the forward angle $\theta_{\mathrm{cm}} \leq 60^{\circ}$ is greatly modified, giving a better reproduction of the $d p$ experimental data. In $T_{21}(\theta)$ at $E_{d}=5-18 \mathrm{MeV}$, dip structure around $\theta_{\text {cm }} \sim 90^{\circ}$ are not sufficiently reproduced in ours calculations. This tendency is also seen in the calculations of Ref. [68], but the values predicted by Alt et al. [30] are too small in this angular region probably because of the too much simplified nuclear interaction (separable potentials). At the maximum point around $\theta_{\mathrm{cm}} \sim 120^{\circ}$, the Coulomb effect remains at $E_{d} \leq 22 \mathrm{MeV}$. For $T_{22}(\theta)$, the Coulomb effect generally raises the minimum point in the low-energy side with $E_{d} \leq 35 \mathrm{MeV}$, which results in good agreement with the experimental data. At $E_{d} \leq 6 \mathrm{MeV}$, the predicted values at the minimum point overshoot the experimental data and are slightly too much reduced. As to this small difference between the theory and the experiment, the comparison with the result in Ref. [68] indicates that the $3 N$ force effect might be important to enhance the magnitudes at the minimum points.

\section{Deuteron breakup processes}

\subsection{Breakup differential cross sections for various kinematical configurations}

A large number of examples for the breakup differential cross sections, predicted by fss2, are already published in Ref. [36] for the neutron-induced $n+d$ reactions without the Coulomb force. In these cases, two neutrons in principle should be detected experimentally in various kinematical configurations. It is customary to classify the three-body breakup kinematics into the following six categories based on the classical (or geometrical) argument:

1. The quasi-free scattering (QFS): one of the nucleons in the final state is at rest in the lab system $\left(\boldsymbol{k}_{\alpha}=0\right)$. 

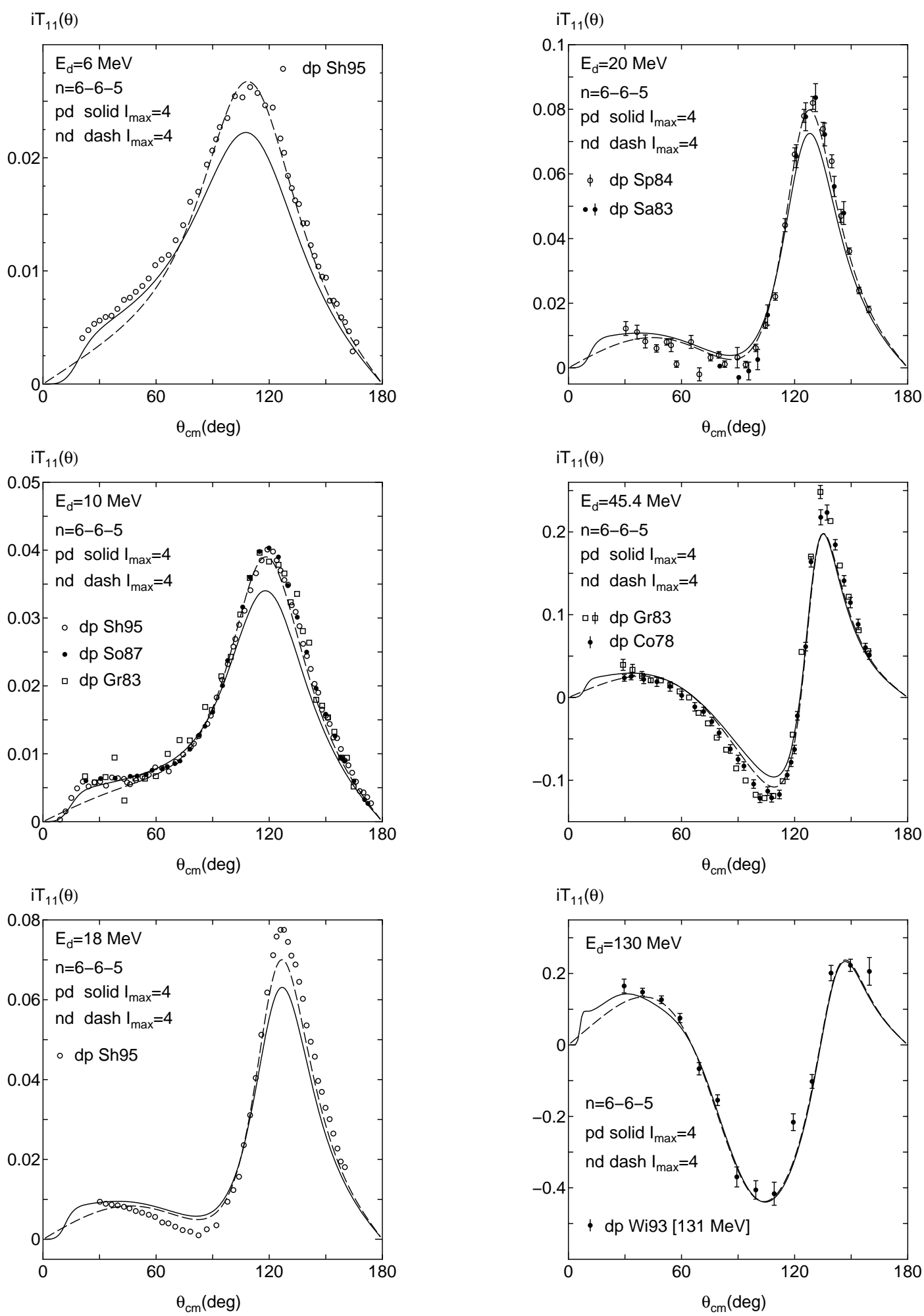

Fig. 10 Vector-type deuteron analyzing power $i T_{11}(\theta)$ for the $n d$ and $p d$ elastic scattering from $E /$ nucleon $=3$ to $65 \mathrm{MeV}$, compared with the experimental data. The experimental data are taken from Refs. [86] for Sh95, [107] for So87, [76] for Gr83, [77] for Sp84, [104] for Sa83, [98] for Co78, and Ref. [108] for Wi93. 

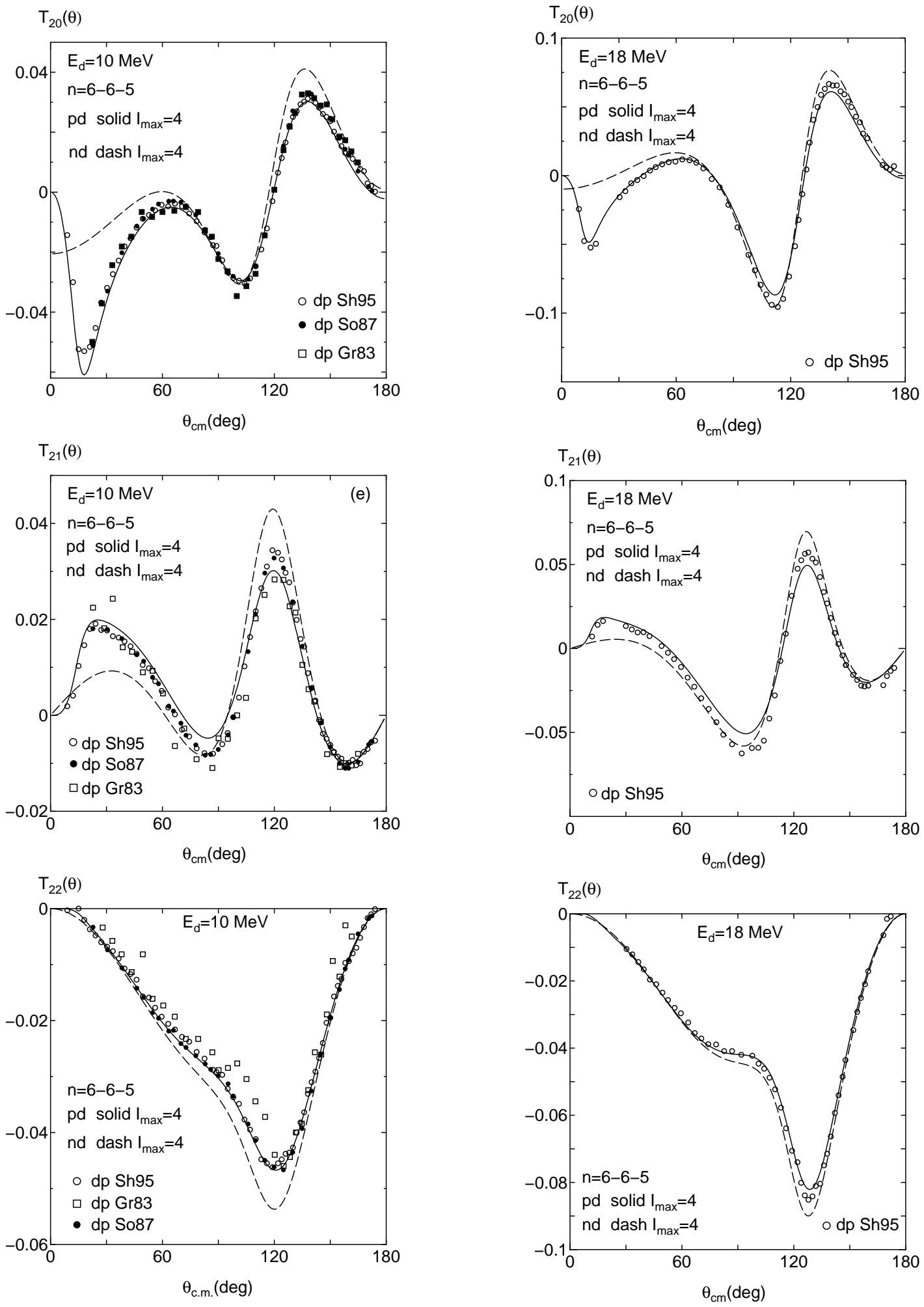

Fig. 11 Tensor-type deuteron analyzing powers $i T_{2 m}(\theta)$ with $m=0,1$ and 2 for the $n d$ and $p d$ elastic scattering at $E /$ nucleon $=5 \mathrm{MeV}$ (left panels) and $9 \mathrm{MeV}$ (right panels), compared with the experimental data. The experimental data are taken from Refs. [86] for Sh95, [107] for So87, and Ref. [76] for Gr83. 

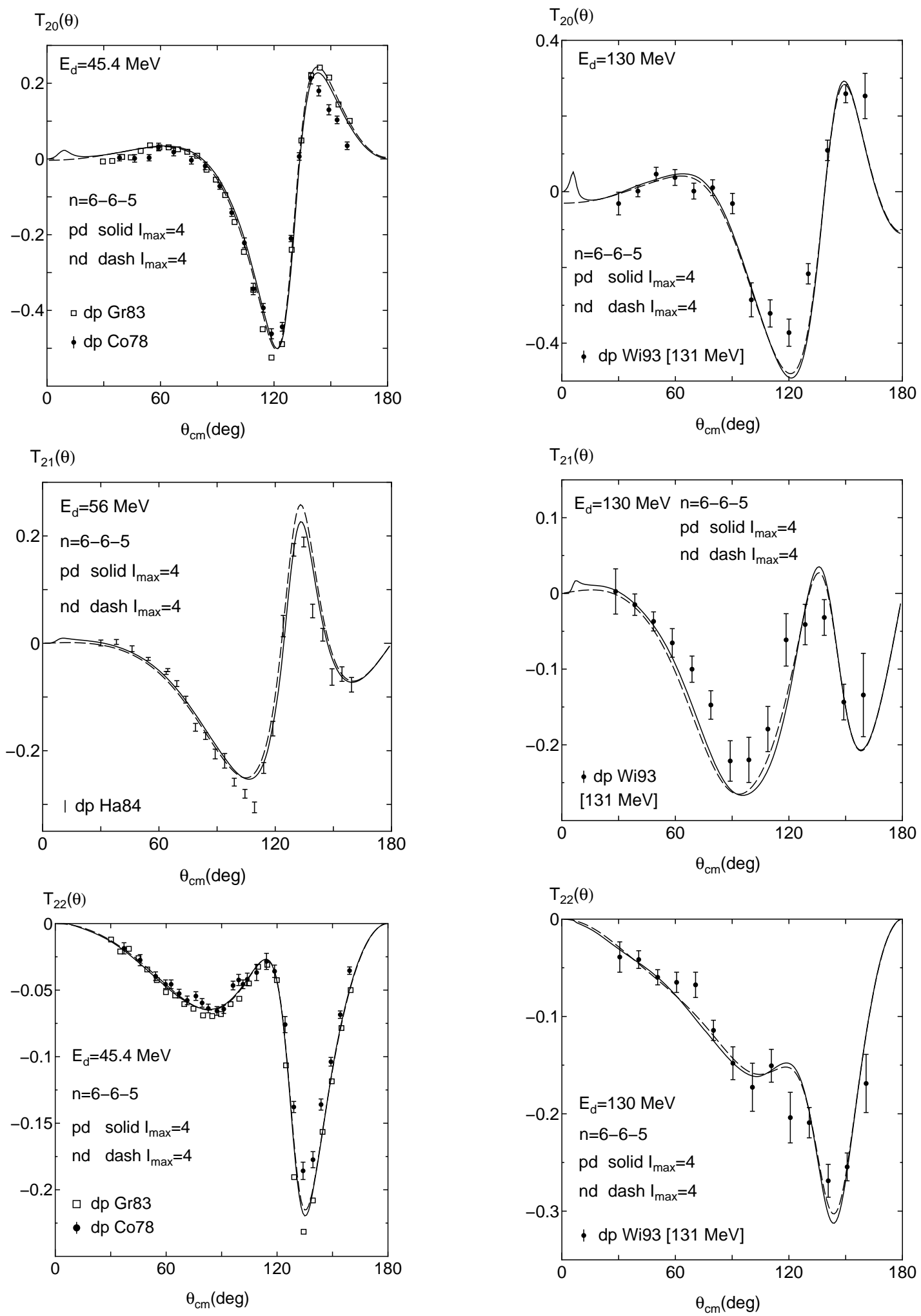

Fig. 12 Same as Fig. 11, but for $E /$ nucleon $=22.7 \mathrm{MeV}\left(T_{20}(\theta)\right.$ and $\left.T_{22}(\theta)\right)$ or $28 \mathrm{MeV}\left(T_{21}(\theta)\right)$ (left panels), and $65 \mathrm{MeV}$ (right panels). The experimental data are taken from Refs. [76] for Gr83, [98] for Co78, [84] for Ha84, and [108] for Wi93. 
2. The final-state interaction (FSI): the relative momentum of the two outgoing nucleons is equal to zero $\left(\boldsymbol{p}_{\alpha}=0\right)$.

3. The collinear configuration (COLL): one of the outgoing nucleons is at rest in the cm system, and the other two have momenta back to back $\left(\boldsymbol{q}_{\alpha}=0\right)$.

4. The symmetric space star configuration (SST): the three nucleons emerge from the reaction point in the cm system, keeping equal momenta with $120^{\circ}$ relative to each other and perpendicular to the beam direction (on the $x-y$ plane in the cm system).

5. The coplanar star configuration (CST): the same as the symmetric space star configuration, but with the three momenta lying on the reaction plane.

6. The non-standard configuration (NS): the other non-specific configurations.

From these configurations, we can roughly estimate which portion of the $N N T$-matrix is responsible at the final stage of the reaction, according to the structure of the direct breakup amplitudes in Eq. (3.41). For example, Ref. [21] argues that the first Born term of the QFS is approximately a product of an on-shell two-nucleon $T$-matrix and the deuteron wave function at zero momentum. It is known that the $3 N$ force effect is rather small for the QFS condition. On the other hand, the collinear configurations are expected to be sensitive to the $3 N$ force intuitively. For the FSI configuration the half-off shell $T$-matrix generates a large peak corresponding to the ${ }^{1} S_{0}$ positive-energy bound state near the zero-energy threshold. It is well known that the $n d$ theoretical calculations in the SST configuration underpredict the experimental values largely, which is called the space star anomaly [113]. It should be noted, however, that the disagreement between the theory and experiment is also seen in some other coplanar star and non-standard configurations.

Actually, many of the experimental data are for the $p+d$ reactions and the Coulomb force should be included to compare the calculated results with experiment, which is particularly important in the $T$-matrix at the final stage of rearrangement in Eq. (3.41). The convergence of the partial-wave expansion is very slow for the screened Coulomb potential with a large cutoff Coulomb radius $\rho$. The half off-shell $T$-matrix for the nuclear plus screened Coulomb potential in Eq. (3.41) begins to oscillate in $p$ when $\rho$ becomes large, and eventually approaches to the full Coulomb result except for the renormalization phase factor. The half-off shell Coulomb $T$-matrix is a smooth function of $p$, but has a sharp singularity at $p=p_{0}$ in the on-shell limit. We therefore need to take a large $I_{\max }$ and enough number of discretization points for the relative momentum $p$ between two protons, especially in the small $p$ region. In this paper, we modify the four-interval separation of the $p$-mesh points with the middle points 1,3 and $6 \mathrm{fm}[22]$ in the no-Coulomb case to five-interval separation with $0.2,0.8$, 2.4 and $5 \mathrm{fm}$ when the Coulomb force is included. We use the same notation $n=n_{1}-n_{2}-n_{3}=5-6-5$ as in $\S 3.1$ of Ref. [22], but the actual number of discretization points is $4 \times 6+5=29$. In the following figures, we show the calculated results with $\rho=8$ (dotted curve), 16 (solid curve) and $20 \mathrm{fm}$ (bold solid curve), together with $I_{\max }=4$. For $\rho=8 \mathrm{fm}$, two different choices of the $p$-mesh points above give almost the same results.

Figure 13 shows the breakup differential cross sections for the reactions $d(p, 2 p) n$ and $d(n, 2 n) p$ with the nucleon incident lab. energy $E_{N}=13 \mathrm{MeV}$. The $n d$ calculations are shown by the dashed curves and should be compared with the experimental data with error bars. We find that the Coulomb effect is rather small in these examples and our results are very similar to the predictions by the meson-exchange potentials, given in Refs. $[21 ; 32 ; 44 ; 114]$. A slight overestimation of the peak in quasi-free scattering (QFS) is reduced by the Coulomb effect and the agreement with the $p d$ experimental data [109] or more recent one [115] from Kyushu university group is improved for $\rho=16 \mathrm{fm}$. However, if we further increase $\rho$ up to $20 \mathrm{fm}$, a catastrophic situation takes place and the peak height is too much suppressed. The $n p$ final state interaction (FSI) peaks are well reproduced. In the collinear (COLL1, COLL2) and coplanar star (CST1) configurations, the nd data [113] agree well with theoretical predictions, but our CST1 result is at least $10-20 \%$ too small. The space star result is located just between the lower $p d$ data and the higher $n d$ data, which is the same feature as other predictions by the meson-exchange potentials. This disagreement of breakup differential cross sections at $E_{n}=13 \mathrm{MeV}$ was reported a long time ago, and is still an unsolved problem called the space star anomaly [113]. 
$\mathrm{d}^{5} \sigma / \mathrm{d} \Omega_{1} \mathrm{~d} \Omega_{2} \mathrm{dS} \quad\left[\mathrm{mb} / \mathrm{MeV} \mathrm{sr^{2 }}\right]$

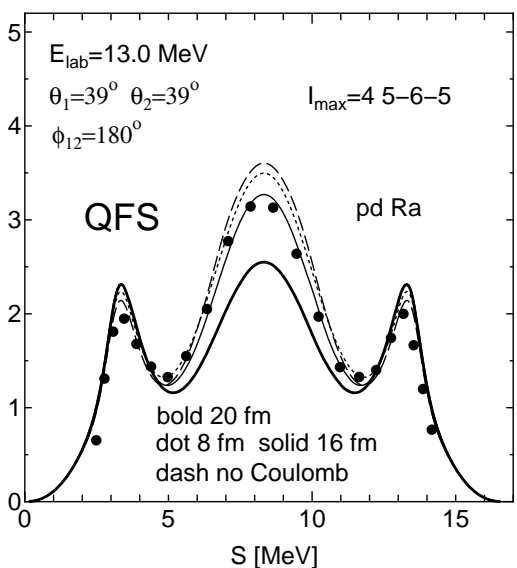

$\mathrm{d}^{5} \sigma / \mathrm{d} \Omega_{1} \mathrm{~d} \Omega_{2} \mathrm{~d} S \quad\left[\mathrm{mb} / \mathrm{MeV} \mathrm{sr}{ }^{2}\right]$

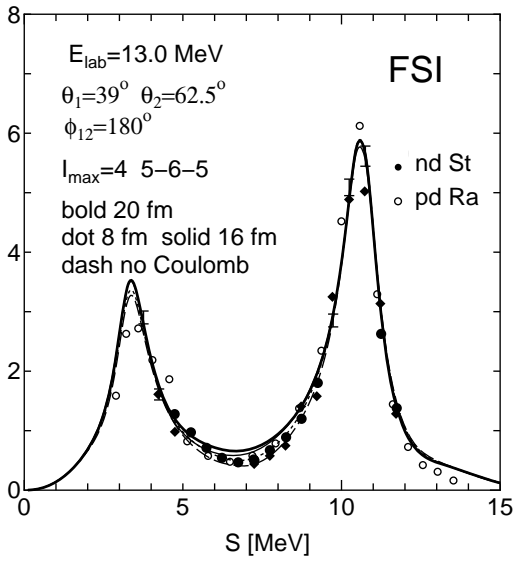

$\mathrm{d}^{5} \sigma / \mathrm{d} \Omega_{1} \mathrm{~d} \Omega_{2} \mathrm{dS} \quad\left[\mathrm{mb} / \mathrm{MeV} \mathrm{sr}^{2}\right]$

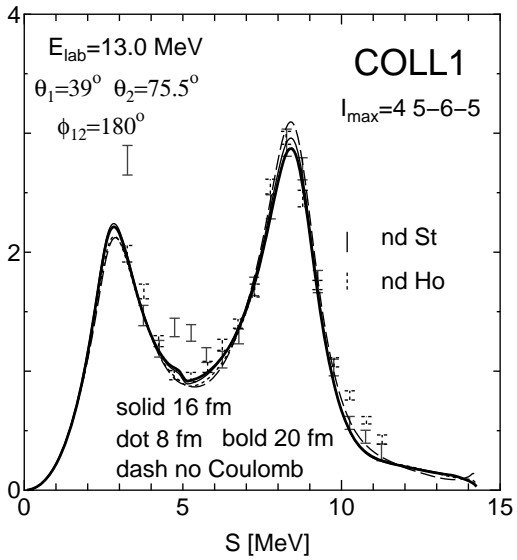

$\mathrm{d}^{5} \sigma / \mathrm{d} \Omega_{1} \mathrm{~d} \Omega_{2} \mathrm{dS} \quad\left[\mathrm{mb} / \mathrm{MeV} \mathrm{sr^{2 } ]}\right.$

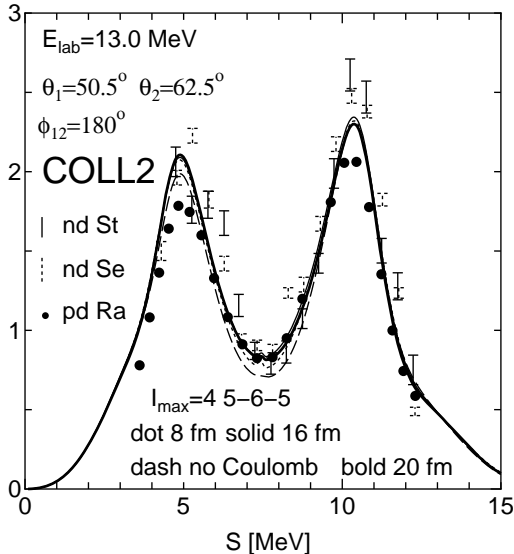

$\mathrm{d}^{5} \sigma / \mathrm{d} \Omega_{1} \mathrm{~d} \Omega_{2} \mathrm{dS} \quad\left[\mathrm{mb} / \mathrm{MeV} \mathrm{sr}{ }^{2}\right]$

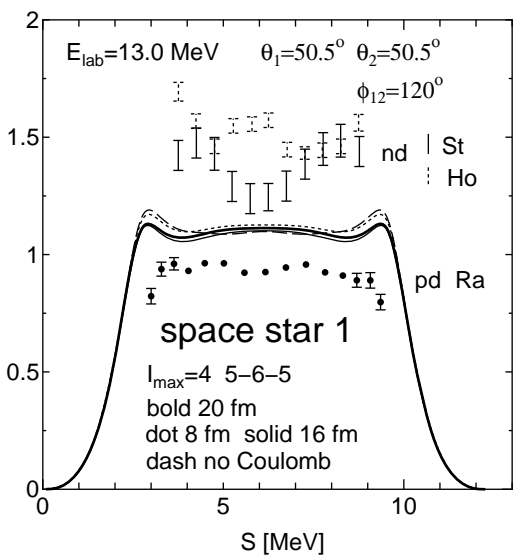

$\mathrm{d}^{5} \sigma / \mathrm{d} \Omega_{1} \mathrm{~d} \Omega_{2} \mathrm{dS} \quad\left[\mathrm{mb} / \mathrm{MeV} \mathrm{sr}^{2}\right]$

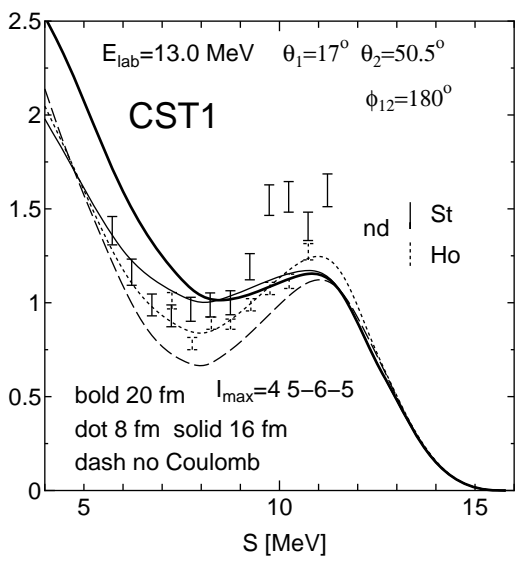

Fig. 13 Breakup differential cross sections for the $n d$ and $p d$ reactions at $E_{N}=13 \mathrm{MeV}$ for the quasi-free scattering (QFS), the final state interaction (FSI), the collinear configurations (COLL1, COLL2), the symmetric space star (space star 1), and the coplanar star (CST1) configurations. The $n d$ result is shown by the dashed curve and the $p d$ results by the dotted $(\rho=8 \mathrm{fm})$, solid $(\rho=16 \mathrm{fm})$ and bold solid $(\rho=20 \mathrm{fm})$ curves. The experimental data are taken from Ref. [109] for the quasi-free scattering, Refs. [109] $(p d)$ and $[110 ; 111 ; 112]$ $(n d)$ for the final state interaction, Refs. [109] $(p d)$ and $[110 ; 111 ; 113](n d)$ for the collinear configurations, Refs. $[110 ; 111 ; 112 ; 113](n d)$ and $[109](p d)$ for the space star configuration, and Refs. [110; 113] for the coplanar star configuration. 

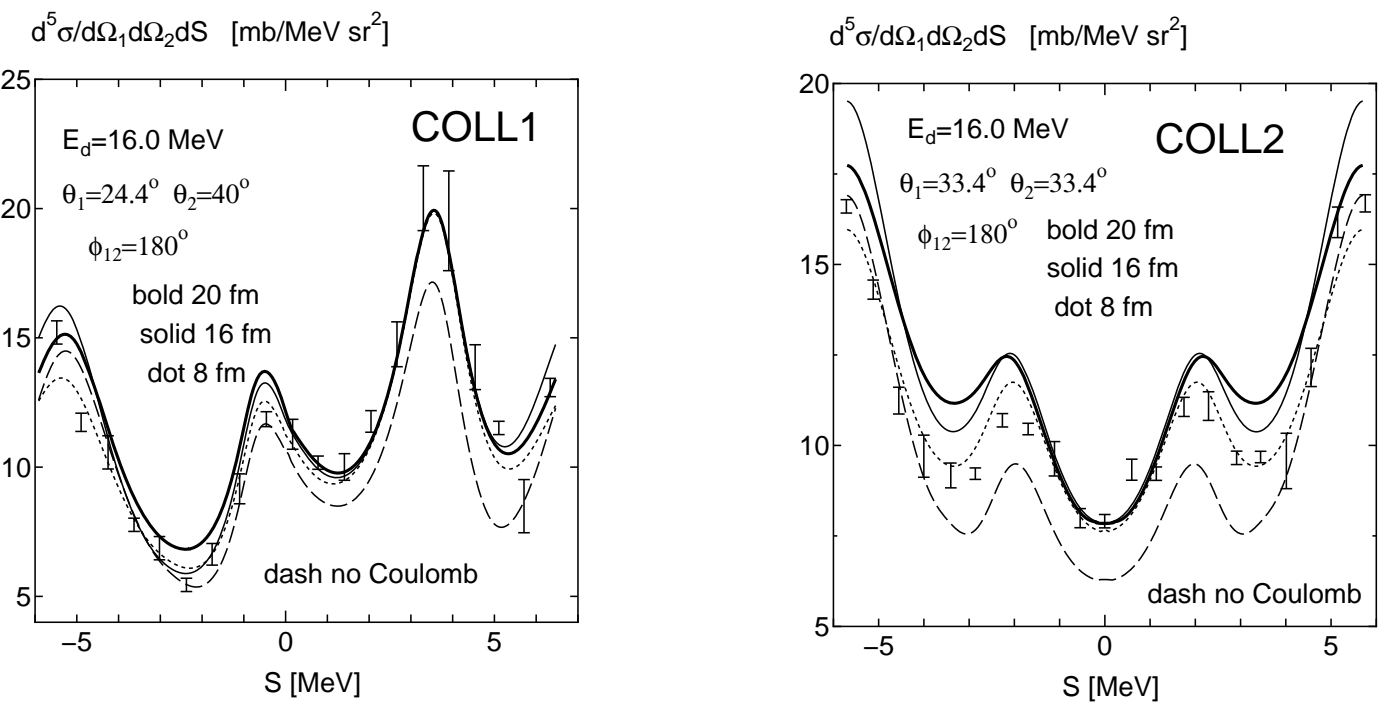

$\mathrm{d}^{5} \sigma / \mathrm{d} \Omega_{1} \mathrm{~d} \Omega_{2} \mathrm{dS} \quad\left[\mathrm{mb} / \mathrm{MeV} \mathrm{sr}{ }^{2}\right]$
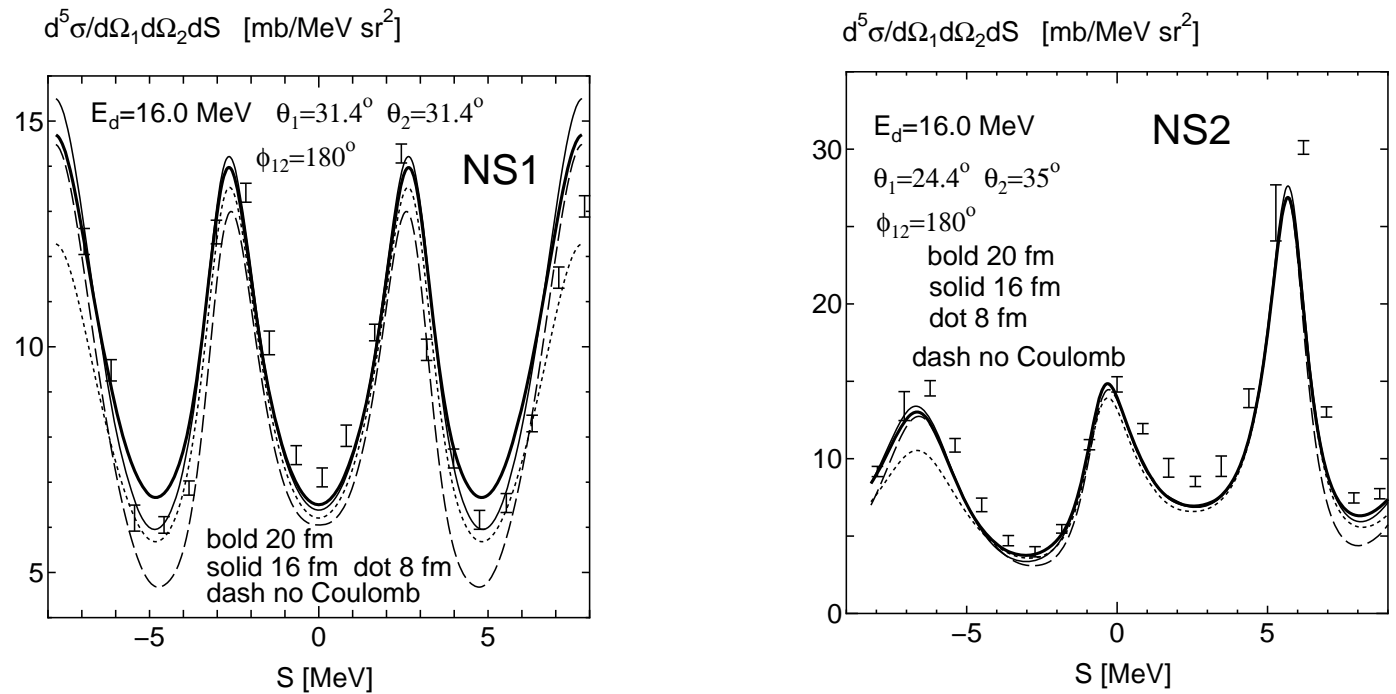

Fig. 14 Breakup differential cross sections for the reaction ${ }^{1} \mathrm{H}(\vec{d}, 2 p) n$ with $E_{d}=16 \mathrm{MeV}$, compared with the experimental data [116]. The cutoff Coulomb radii, $\rho=8$ (dotted), 16 (solid) and $20 \mathrm{fm}$ (bold solid curves), together with $I_{\max }=4$ are used. No-Coulomb results are shown by the dashed curves.

\section{$5.2{ }^{1} \mathrm{H}(\vec{d}, 2 p) n$ reaction at $E_{d}=16 \mathrm{MeV}$}

Figure 14 shows the $n d$ and $p d$ breakup differential cross sections at the two collinear (COLL1, COLL2) and two nonstandard (NS1, NS2) configurations, compared with the $d p$ experimental data of Correll's et al. [116] for the reaction ${ }^{1} \mathrm{H}(\vec{d}, 2 p) n$ at $E_{d}=16 \mathrm{MeV}$. This corresponds to the nucleon-induced breakup reaction at the nucleon incident energy of $8 \mathrm{MeV}$. The $n d$ breakup differential cross sections are shown by dashed curves and the experimental data should be compared with other curves. The starting point $S=0$ is chosen as each collinear point or the nearest point, as discussed Appendix B of Ref. [36]. In general, the agreement with experimental data is good, although not perfect especially for COLL2. If we compare our $n d$ results (dashed curves) with the predictions by meson-exchange predictions in Ref. [21], we find that they are very similar to each other. The Coulomb effect clearly improves the agreement with the experimental data in collinear (COLL1, COLL2) and nonstandard (NS1) configurations, although the results by $\rho=20 \mathrm{fm}$ sometimes overshoot the experimental data. 


\subsection{Comparison with the KVI data for the ${ }^{1} \mathrm{H}(\vec{d}, 2 p) n$ reaction at $E_{d}=130 \mathrm{MeV}$}

Very accurate KVI data for the ${ }^{1} \mathrm{H}(\vec{d}, 2 p) n$ reaction with $E_{d}=130 \mathrm{MeV}[117 ; 118]$ are compared with our predictions in Fig. 15. The $n d$ results are shown by the dashed curves. For the $d p$ calculations, the cutoff parameter $\rho$ for the Coulomb force is chosen to be $8 \mathrm{fm}$ (dotted curve), $16 \mathrm{fm}$ (solid curve) and $20 \mathrm{fm}$ (bold solid curve). Since the starting positions of $S=0$ are not the same between our calculation and the experiment, we shift the $S$-axis of the calculated result by the amount denoted in each figure. The dip structure seen at the $p p$ final state interaction for the first panel PL-1 $\left(\theta_{1}=\theta_{2}=13^{\circ}\right.$ and $\phi_{12}=20^{\circ}$ ) is properly reproduced when we choose the cutoff parameter $\rho \geq 16 \mathrm{fm}$. On the other hand, the improvement by the Coulomb effect with $\rho=8 \mathrm{fm}$ is spoiled when $\rho$ is increased to $16 \mathrm{fm}$ or 20 $\mathrm{fm}$ in PL-2. In other cases, we find that the $p d$ calculations with the Coulomb effect generally improve the agreement with experiment. For the large azimuthal angles $\phi_{12} \sim 180^{\circ}$, the underestimation of the breakup differential cross sections may not be only due to the flaw in the Coulomb treatment. We have also compared our calculations with a large amount of the systematic data of the KVI group [117], although they are not shown in this paper. The overall agreement between the theory and the experiment is obtained.

The vector-type and tensor-type analyzing powers of the deuteron in the breakup differential cross sections are shown in Figs. 16 and 17, respectively. No-Coulomb results denoted by the dashed curves almost reproduce the experimental data on the whole, but some uncomfortable oscillations develop when the Coulomb cutoff radius is increased up to $\rho=20 \mathrm{fm}$. This is particularly serious for configurations of the $p p$ final-state interaction with $\theta_{1} \sim \theta_{2}$ and $\phi_{12}=0$ in Fig. 17, where a great accuracy around the small $p \sim p_{0}$ is required as discussed in $\S 5.1$.

\section{Summary and outlook}

In this series of studies $[22 ; 23 ; 37 ; 36 ; 11]$, we have applied the quark-model (QM) baryon-baryon interaction fss 2 to the neutron-deuteron $(n d)$ and the proton-deuteron $(p d)$ scattering in the Faddeev formalism for composite particles. The main motivation is to investigate the off-shell effect of the QM nucleon-nucleon $(N N)$ interaction, in which the short-range repulsion is described by the nonlocalities of the quark exchange kernel in the resonating-group method (RGM), assuming the naive three-quark structure of nucleons. The practical calculations are carried out using the 15-point Gaussian nonlocal potential constructed from fss2, which is tailored to reproduce the converged triton binding energy of fss2 with an accuracy of $15 \mathrm{keV}$ and the $N N$ phase-shift parameters with a difference of less than $0.1^{\circ}$ $[23 ; 7]$. The potential keeps all the nonlocal effects of the original fss2, including the energy-dependent term of the QM RGM. The energy dependence is eliminated by the standard off-shell transformation utilizing the square root of the normalization kernel for two three-quark clusters [2]. It is extremely important to deal with this energy dependence properly, since an extra nonlocal kernel generated from this procedure is crucial to reproduce all the elastic scattering observables below $E_{n} \leq 65 \mathrm{MeV}$ [22; 37].

In order to compare our results with many accurate experimental data of the $p d$ scattering, we have incorporated the Coulomb force in an approximate way of using the screened Coulomb potential. Based on the two-potential formula, we have extended the Vincent and Phatak method [34] for the sharply cut-off Coulomb problem to the $p d$ elastic scattering, using a connection condition for the $K$-matrix derived from the asymptotic waves [11]. This method gives reasonable results for various scattering observables with a rather moderate value for the Coulomb cutoff radius around $\rho \sim 8$ $9 \mathrm{fm}$. The nuclear - Coulomb interference in the forward angular region is correctly reproduced for the differential cross sections and analyzing powers. The breakup differential cross sections and the deuteron analyzing powers of the $d p$ and $p d$ reactions are also calculated with the same solutions of the AGS equation as the elastic scattering, by incorporating the half off-shell $T$-matrix for the nuclear plus screened Coulomb potential in the final $p p$ channel. It turns out that, in the kinematical configurations involving the $p p$ final-state interaction, a large $\rho$ value greater than $16 \mathrm{fm}$ is required to reproduce an intricate behavior of the differential cross sections and analyzing powers. Accurate calculations including enough number of partial waves and low-momentum discretization points for the $p p$ relative motion are necessary to obtain reliable results.

We have found that our calculations reproduce almost all the results for the breakup differential cross sections, predicted by the meson-exchange potentials, including the disagreement with the ex- 

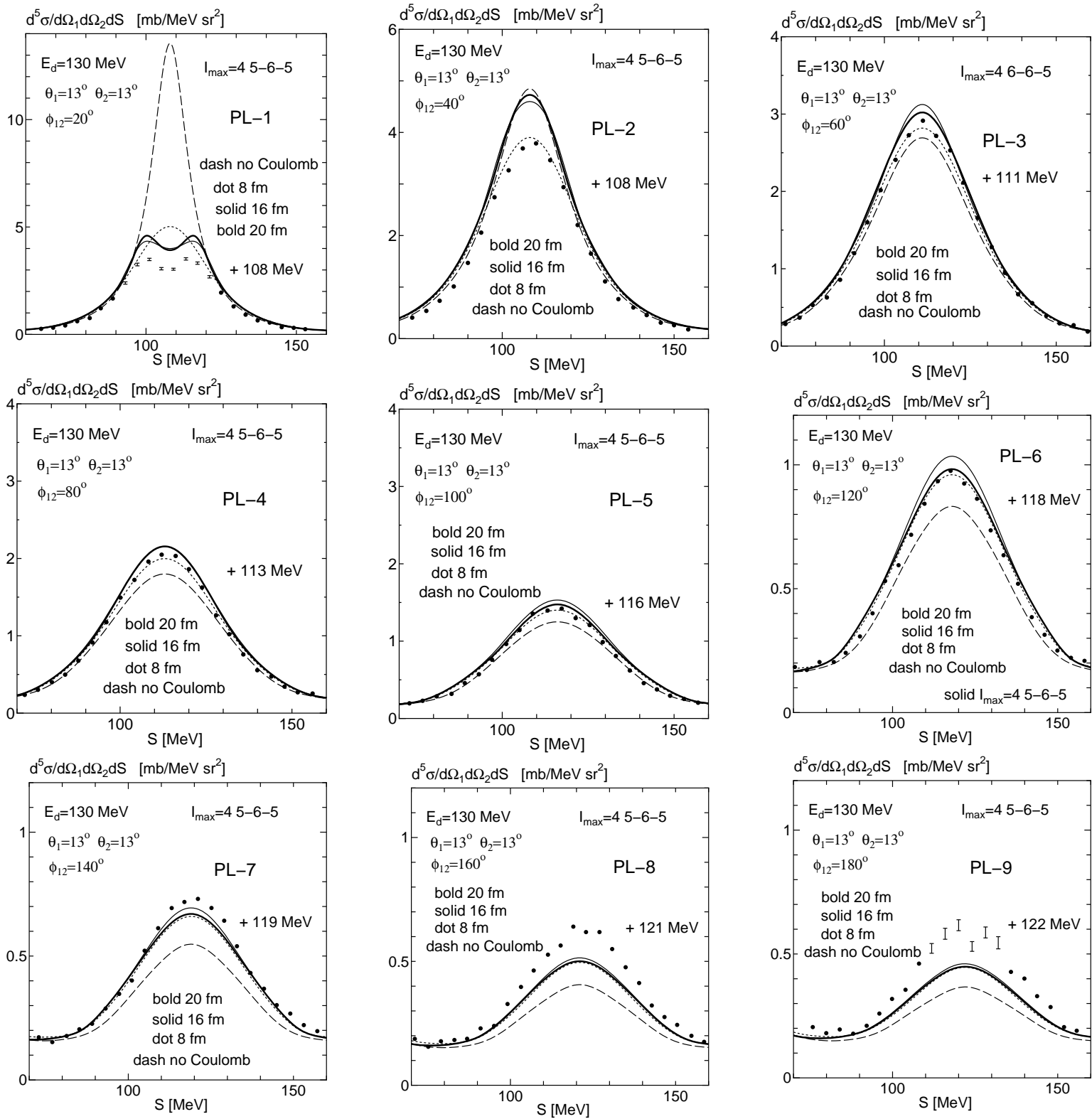

Fig. 15 Breakup differential cross sections for the reaction ${ }^{1} \mathrm{H}(\vec{d}, 2 p) n$ at $E_{d}=130 \mathrm{MeV}$, compared with the experimental data [118]. The $n d$ results are shown by the dashed curves. For the $d p$ calculations, the Coulomb cutoff parameter $\rho$ is chosen to be 8 (dotted), 16 (solid) and $20 \mathrm{fm}$ (bold solid curves). Here, the kinematical conditions are $\theta_{1}=\theta_{2}=13^{\circ}$, and $\phi_{12}$ are changed from $20^{\circ}$ to $180^{\circ}$ with a step of $20^{\circ}$. The calculated curves are shifted by about $100 \mathrm{MeV}$ with respect to $S$ to fit the experimental origin.

periment. This feature is probably related to the structure of the direct breakup amplitudes in Eq. (3.41). First, they are constrained by the elastic scattering amplitudes $f_{\left(\ell^{\prime} S_{c}^{\prime}\right)\left(\ell S_{c}\right)}^{J}$ in the initial stage. In the final stage of reactions, only the half-off shell two-nucleon $T$-matrix appears owing to the energy conservation for outgoing nucleons. The effect of the completely off-shell $T$-matrix therefore appears only at the stage of solving the basic AGS equations for $\left\langle p, q, \gamma|\widetilde{Q}| \phi ;\left(\ell^{\prime} S_{c}^{\prime}\right) J\right\rangle$, for which the present investigations imply that the difference between our QM $N N$ interaction and the meson-exchange potentials is rather minor. On the whole, the agreement with the experimental data is fair, but there exist discrepancies in some particular kinematical configurations, which are commonly found for both our predictions and meson-exchange predictions. In particular, the space star anomaly of $13 \mathrm{MeV} n d$ 

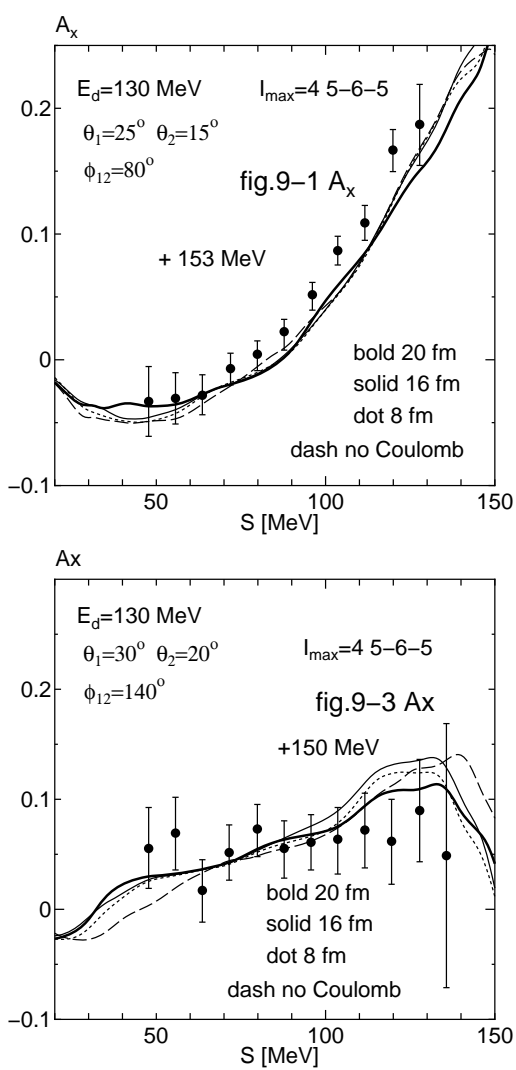
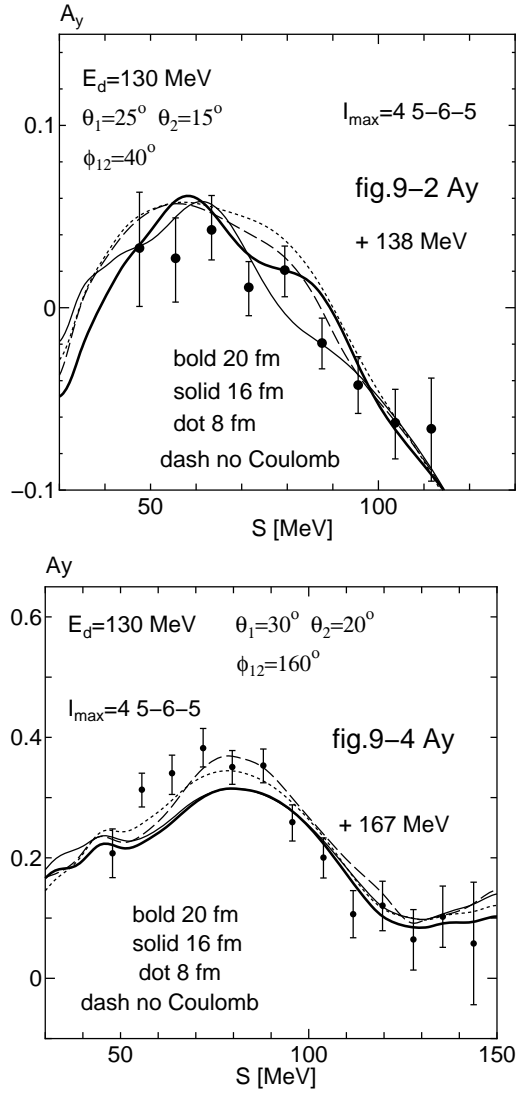

Fig. 16 Vector analyzing power of the deuteron for the reaction ${ }^{1} \mathrm{H}(\vec{d}, 2 p) n$ with $E_{d}=130 \mathrm{MeV}$, compared with the experimental data [119]. The cutoff Coulomb radii, $\rho=8$ (dotted), 16 (solid) and $20 \mathrm{fm}$ (bold solid curves), together with $I_{\max }=4$ are used. No-Coulomb results are shown by the dashed curves. The calculated curves are shifted by about $150 \mathrm{MeV}$ with respect to $S$ to fit the experimental origin.

and $p d$ scattering is not improved even in our model. There are major disagreements in the breakup differential cross sections in some of the coplanar and nonstandard configurations. Since these large disagreements can be resolved neither by the Coulomb effect nor by the introduction of the $3 N$ force, systematic studies from more basic viewpoints of the $N N$ interaction are still needed both experimentally and theoretically.

In spite of the apparent disagreement between the theory and the experiment in some of the breakup observables, our QM $N N$ interaction fss2 is still very successful in reproducing the essential features of almost all other experimental data in the three-nucleon system without reinforcing it with the three-body force. These include:

1) a nearly correct binding energy of the triton [3],

2) reproduction of the doublet and quartet $S$-wave scattering lengths, ${ }^{2} a$ and ${ }^{4} a$ [23],

3) low-energy differential cross sections of the $p d$ elastic scattering up to $E_{p} \sim 35 \mathrm{MeV}$ at the diffraction minimum points [22],

4) improved maximum heights of the nucleon analyzing power $A_{y}(\theta)$ in the low-energy region $E_{n} \leq 25$ $\mathrm{MeV}$, although still insufficient [37],

5) breakup differential cross sections with many kinematical configurations [36].

Many of these improvements are related to the sufficiently attractive $n d$ interaction in the ${ }^{2} S$ channel, in which the strong distortion effect of the deuteron is very sensitive to the treatment of the shortrange repulsion of the $N N$ interaction. In our QM $N N$ interaction, this part is described by the quark exchange kernel of the color-magnetic quark-quark interaction. In the strangeness sector involving the $\Lambda N$ and $\Sigma N$ interactions, the effect of the Pauli repulsion on the quark level appears in some baryonic 

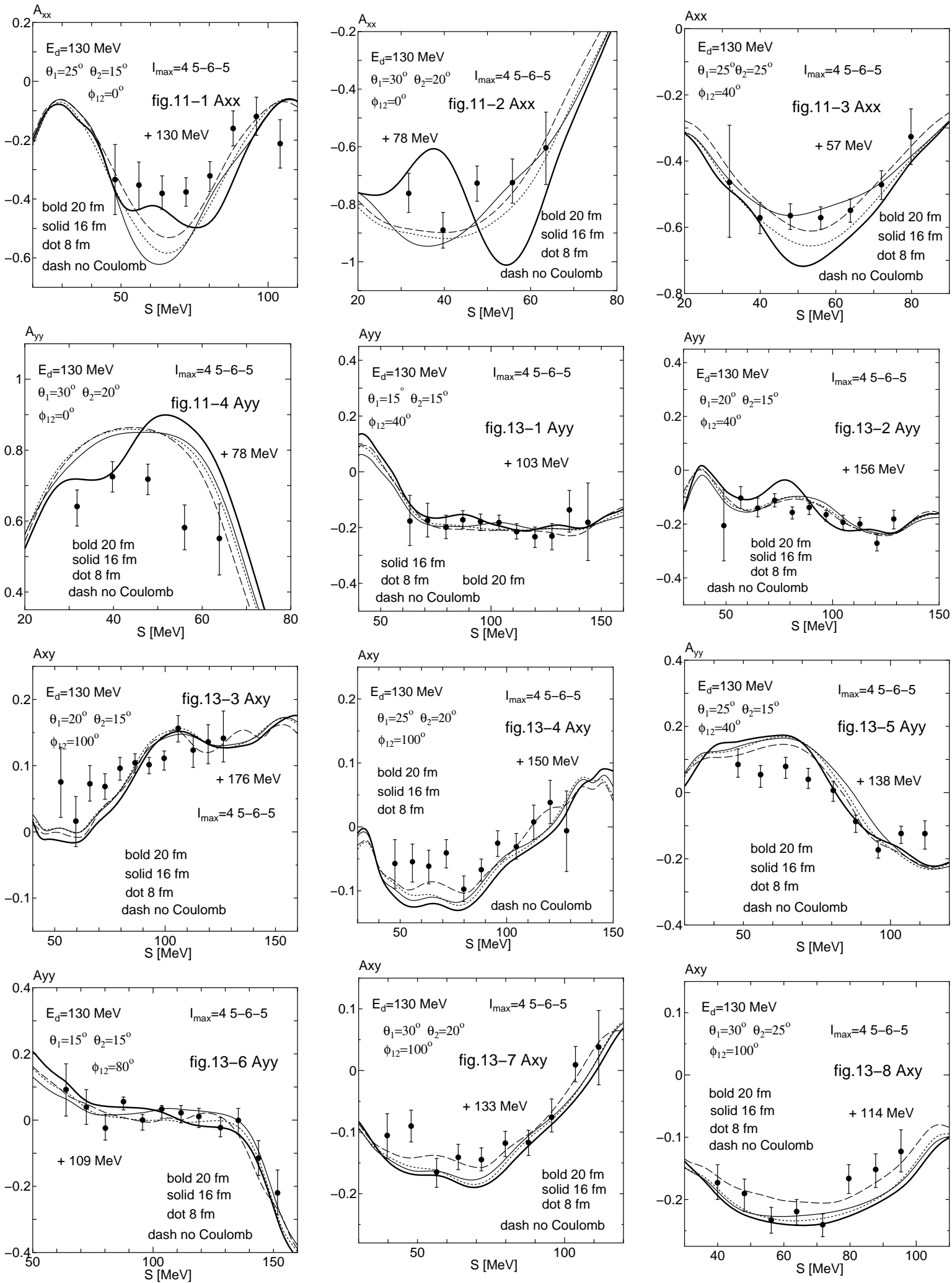

Fig. 17 Same as Fig. 16, but for the tensor analyzing powers of the deuteron. 
channels. It is therefore interesting to study $\Sigma^{ \pm}$-deuteron scattering in the present framework to find the repulsive effect directly related to the quark degree of freedom. Such an experiment is being planned at the J-PARC facility [120].

Acknowledgements The authors would like to thank Professors H. Witała, K. Miyagawa, H. Kamada and S. Ishikawa for many useful comments. We also thank Professor K. Sagara and the Kyushu University group for providing us accurate experimental data for the $p d$ elastic scattering and results of systematic analyses on the space-star anomaly and quasi-free scattering. This work was supported by a Grant-in-Aid for Scientific Research (C) from the Japan Society for the Promotion of Science (JSPS) (Grant No. 23540302), and by a Grant-in-Aid for the Global COE Program "The Next Generation of Physics, Spun from Universality and Emergence" from the Ministry of Education, Culture, Sports, Science and Technology (MEXT) of Japan. It was also supported by core-stage backup subsidies from Kyoto University. The numerical calculations were carried out on SR16000 at YITP in Kyoto University and on the high-performance computing system Intel Xeon X5680 at RCNP in Osaka University.

\section{References}

1. Y. Fujiwara, Y. Suzuki, and C. Nakamoto. Baryon-baryon interactions in the $S U_{6}$ quark model and their applications to light nuclear systems. Prog. Part. Nucl. Phys., 58:439-520, 2007.

2. Y. Suzuki, H. Matsumura, M. Orabi, Y. Fujiwara, P. Descouvemont, M. Theeten, and D. Baye. Local versus nonlocal $\alpha \alpha$ interaction in $3 \alpha$ description of ${ }^{12}$ C. Phys. Lett. B659:160-164, 2008.

3. Y. Fujiwara, Y. Suzuki, M. Kohno, and K. Miyagawa. Addendum: Triton and hypertriton binding energies calculated from $S U_{6}$ quark-model baryon-baryon interactions. Phys. Rev. C, 77:027001, 2008.

4. A. Nogga, H. Kamada, and W. Glöckle. Modern nuclear force predictions for the $\alpha$ particle. Phys. Rev. Lett., 85:944, 2000.

5. Y. Fujiwara, Y. Suzuki, K. Miyagawa, M. Kohno, and H. Nemura. Redundant components in the $3 \alpha$ Faddeev equation using the $2 \alpha$ RGM kernel. Prog. Theor. Phys., 107:993, 2002.

6. E. O. Alt, P. Grassberger, and W. Sandhas. Reduction of the three-particle collision problem to multichannel two-particle Lippmann-Schwinger equations. Nucl. Phys., B2:167, 1967.

7. K. Fukukawa, Y. Fujiwara, and Y. Suzuki. Gaussian nonlocal potentials for the quark-model baryonbaryon interactios. Mod. Phys. Lett., A24:1035-1038, 2009

8. H. P. Noyes. New nonsingular integral equation for two-particle scattering. Phys. Rev. Lett., 15:538, 1965.

9. K. L. Kowalski. Off-shell equations for two-particle scattering. Phys. Rev. Lett., 15:798; 908 (E), 1965.

10. H. Witała, Th. Cornelius, and W. Glöckle. Elastic scattering and break-up process in the $n$ - $d$ system. Few-Body Systems, 3:123, 1988

11. Y. Fujiwara and K. Fukukawa. A practical method to solve cut-off Coulomb problems in the momentum space - Application to the Lippmann-Schwinger resonating-group method and the $p d$ elastic scattering -. Prog. Theor. Phys., 128:301, 2012.

12. Y. Fujiwara, T. Fujita, M. Kohno, C. Nakamoto, and Y. Suzuki. Resonating-group study of baryon-baryon interactions for the complete baryon octet: $N N$ interaction. Phys. Rev. C, 65:014002, 2002.

13. Y. Fujiwara, M. Kohno, C. Nakamoto, and Y. Suzuki. Interactions between octet baryons in the $S U_{6}$ quark model. Phys. Rev. C, 64:054001, 2001.

14. R. A. Arndt. Scattering Analysis Interactive Dial-up (SAID). Virginia Polytechnic Institute, Blacksburg, Virginia, Private Communication.

15. Y. Fujiwara, M. Kohno, T. Fujita, C. Nakamoto, and Y. Suzuki. Lippmann-Schwinger resonating-group formalism for $N N$ and $Y N$ interactions in an $S U_{6}$ quark model. Prog. Theor. Phys., 103:755-794, 2000.

16. Y. Fujiwara, H. Nemura, Y. Suzuki, K. Miyagawa, and M. Kohno. Three-cluster equation using two-cluster RGM kernel. Prog. Theor. Phys., 107:745, 2002.

17. M. Kohno, Y Fujiwara, T. Fujita, C. Nakamoto, and Y. Suzuki. Hyperon single-particle potentials calculated from $S U_{6}$ quark-model baryon-baryon interactions. Nucl. Phys., A674:229-245, 2000.

18. Y. Fujiwara, M. Kohno, C. Nakamoto, and Y. Suzuki. G-matrix equation in the quark-model resonatinggroup method for baryon-baryon interaction. Prog. Theor. Phys., 104:1025-1040, 2000.

19. Y. Fujiwara. QMPACK homepage, http://qmpack.homelinux.com/ qmpack/index.php.

20. W. Glöckle. The Qunatum Mechanical Few-Body Problem, Texts and Monographs in Physics. Springer, Berlin, Berlin, 1983.

21. W. Glöckle, H. Witała, D. Hüber, H. Kamada, and J. Golak. The three nucleon continuum: achievements, challenges and applications. Phys. Rep., 274:107, 1996.

22. Y. Fujiwara and K. Fukukawa. Quark-model baryon-baryon interaction applied to neutron-deuteron scattering. I - Noyes-Kowalski approach to the AGS equations - . Prog. Theor. Phys., 124:433, 2010.

23. K. Fukukawa and Y. Fujiwara. Effective-range expansion of the nd scattering studied by a quark-model nonlocal gaussian potential. Prog. Theor. Phys., 125:957, 2011.

24. R. G. Seyler. Polarization from scattering polarized spin-1/2 on unpolarized spin-1 particles. Nucl. Phys., A124:253, 1969.

25. W. Glöckle, G. Hasberg, and A. R. Neghabian. Numerical treatment of few body equations in momentum space by the spline method. Z. Phys., A305:217, 1982. 
26. Ch. Elster H. Liu and W. Glöckle. Three-body scattering at intermediate energies. Phys. Rev. C, 72:054003, 2005 .

27. E. O. Alt, W. Sandhas, H. Zankel, and H. Ziegelmann. Coulomb corrections in proton-deuteron scattering. Phys. Rev. Lett., 37:1537, 1976.

28. E. O. Alt and W. Sandhas. Scattering amplitudes and integral equations for the collision of two charged composite particles. Phys. Rev. C, 21:1733, 1980.

29. E. O. Alt, W. Sandhas, and H. Ziegelmann. Calculation of proton-deuteron phase parameters including the Coulomb force. Nucl. Phys., A445:429, 1985.

30. E. O. Alt, A. M. Mukhamedzhanov, M. M. Nishonov, A. I. Sattarov, and W. Sandhas. Proton-deuteron elastic scattering from 2.5 to $22.7 \mathrm{MeV}$. Phys. Rev. C, 65:064613, 2002.

31. A. Deltuva, A. C. Fonseca, and P. U. Sauer. Momentum-space treatment of the Coulomb interaction in three-nucleon reactions with two protons. Phys. Rev. C, 71:054005, 2005.

32. A. Deltuva, A. C. Fonseca, and P. U. Sauer. Momentum-space description of three-nucleon breakup reactions including the Coulomb interaction. Phys. Rev. C, 72:054004, 2005.

33. A. Deltuva, A. C. Fonseca, A. Kievsky, S. Rosati, P. U. Sauer, and M. Viviani. Benchmark calculation for proton-deuteron elastic scattering observables including the Coulomb interaction. Phys. Rev. C, 71:064003, 2005

34. C. M. Vincent and S. C. Phatak. Accurate momentum-space method for scattering by nuclear and Coulomb potential. Phys. Rev. C, 10:391, 1974.

35. G. G. Ohlsen. Kinematic relations in reactions of the form $\mathrm{a}+\mathrm{b} \rightarrow \mathrm{c}+\mathrm{d}+\mathrm{e}$. Nucl. Instrum. Methods, $37: 240,1965$

36. Y. Fujiwara and K. Fukukawa. Quark-model baryon-baryon interaction applied to neutron-deuteron scattering. III - Breakup differential cross sections —. Prog. Theor. Phys., 125:779, 2011.

37. K. Fukukawa and Y. Fujiwara. Quark-model baryon-baryon interaction applied to neutron-deuteron scattering. II — Polarization observables of the elastic scattering —. Prog. Theor. Phys., 125:957, 2011.

38. A. Kievsky, S. Rosati, W. Tornow, and M. Viviani. Critical comparison of experimental data and theoretical predictions for $n$ - $d$ scattering below the breakup threshold. Nucl. Phys., A607:402, 1996.

39. Z. M. Chen, W. Tornow, and A. Kievsky. Extension of proton-deuteron phase-shift analysis to $E_{p}=22.7$ $\mathrm{MeV}$ and ${ }^{4} P_{J}$ phase shifts. Few-Body Systems, 35:15, 2004.

40. W. Tornow and H. Witała. Evaluation of the three-nucleon analyzing power puzzle. Nucl. Phys., A637:280 1998.

41. W. Tornow, A. Kievsky, and H. Witała. Improved proton-deuteron phase-shift analysis above the deuteron-breakup threshold and the three-nucleon analyzing power puzzle. Few-Body Systems, 32:53, 2002.

42. A. Kievsky, J. I. Friar, G. L. Payne, S. Rosati, and M. Viviani. Phase shifts and mixing parameters for low-energy proton-deuteron scattering. Phys. Rev. C, 63:064004, 2001.

43. S. Ishikawa. Low-energy proton-deuteron scattering with a Coulomb-modified Faddeev equation. FewBody Systems, 32:229, 2003.

44. S. Ishikawa. Coordinate space proton-deuteron scattering calculations including Coulomb force effects. Phys. Rev. C, 80:054002, 2009.

45. L. M. Delves. Low-energy photodisintegration of ${ }^{3} \mathrm{He}$ and neutron-deuteron scattering. Phys. Rev., $118: 1318,1960$

46. G. Barton and A. C. Phillips. Neutron-deuteron scattering near threshold: on-shell two-body approach. Nucl. Phys., A132:97, 1969.

47. A. S. Reiner. On the anomalous effective range expansion for nucleon-deuteron scattering in the $S=1 / 2$ state. Phys. Lett., B28:387, 1969.

48. J. S. Whiting and M. G. Fuda. Pole in $k \cot \delta$ for doublet, $s$-wave, $n$ - $d$ scatteing. Phys. Rev. $C, 14: 18$, 1976.

49. J. L. Friar, D. Hüber, and U. van Kolck. Chiral symmetry and three-nucleon forces. Phys. Rev. C, 59:53, 1999.

50. S. A. Coon and H. K. Han. Reworking the Tucson-Melbourne three-nucleon potential. Few-Body Systems, $30: 131,2001$.

51. H. Witała, A. Nogga, H. Kamada, W. Glöckle, J. Golak, and R. Skibiński. Modern nuclear force predictions for the neutron-deuteron scattering lengths. Phys. Rev. C, 68:034002, 2003

52. W. Dilg, L. Koester, and W. Nistler. The neutron-deuteron scattering lengths. Phys. Lett., B36:208, 1971.

53. L. Schlessinger. Use of analyticity in the calculation of nonrelativistic scattering amplitudes. Phys. Rev., $167: 1411,1968$

54. A. C. Phillips. Consistency of the low-energy three-nucleon observables and separable interaction model. Nucl. Phys., A107:209, 1968.

55. P. Schwarz, H. O. Klages, P. Doll, B. Haesner, J. Wilczynski, B. Zeitnitz, and J. Kecskemeti. Elastic neutron-deuteron scattering in the energy range from $2.5 \mathrm{MeV}$ to $30 \mathrm{MeV}$. Nucl. Phys., A398:1, 1983.

56. J. D. Seagrave, J. C. Hopkins, D. R. Dixon, Jr. P. W. Keaton, E. C. Kerr, A. Niiler, R. H. Sherman, and R. K. Walter. Elastic scattering and polarization of fast neutrons by liquid deuterium and tritium. Ann. of Phys., 74:250, 1972

57. G. Pauletta and F. D. Brooks. Cross sections for the $n+d$ breakup reaction. Nucl. Phys., A255:267, 1975.

58. M. Holmberg. The $(n, 2 n)$ cross section of ${ }^{2} \mathrm{H}$ in the energy region $4.0-6.5 \mathrm{MeV}$. Nucl. Phys., A129:327, 1969. 
59. H. C. Catron, M. D. Goldberg, R. W. Hill, J. M. LeBlanc, J. P. Stoering, C. J. Taylor, and M. A. Williamson. Deuterium and beryllium $(n, 2 n)$ cross sections between 6 and $10 \mathrm{MeV}$. Phys. Rev., 123:218, 1961.

60. J. H. Gibbons and R. L. Macklin. Total neutron yields from light elements under proton and alpha bombardment. Phys. Rev., 114:571, 1959.

61. A. Kievsky, C. R. Brune, and M. Viviani. Total cross section for $p$ - $d$ breakup below $30 \mathrm{MeV}$. Phys. Lett., B480:250, 2000.

62. R. F. Carlson, P. Doherty, D. J. Margaziotis, I. Slaus, S. Y. Tin, and W. T. H. van Oers. Proton-deuteron total reaction cross-sections in the energy range $(20-50) \mathrm{MeV}$. Lettere Al Nuovo Cimento, 8:319, 1973.

63. J. M. Clement, P. Stoler, C. A. Goulding, and R. W. Fairchild. Hydrogen and deuterium total neutron cross sections in the MeV region. Nucl. Phys., A183:51, 1972.

64. J. D. Seagrave and R. L. Henkel. Total cross section of deuterium for neutrons from $0.2 \mathrm{MeV}$ to $22 \mathrm{MeV}$. Phys. Rev., 98:666, 1955.

65. T. W. Phillips, B. L. Berman, and J. D. Seagrave. Neutron total cross section for tritium. Phys. Rev. C, $22: 384,1980$

66. R. G. Nuckolls, C. L. Bailey, W. E. Bennett, T. Bergstralh, H. T. Richards, and J. H. Williams. The total scattering cross sections of deuterium and oxygen for fast neutrons. Phys. Rev., 70:805, 1946.

67. H. B. Willard, J. K. Bair, and C. M. Jones. n-d scattering: a search for effects due to the dineutron. Phys. Lett., 9:339, 1964.

68. A. Kievsky, M. Viviani, and S. Rosati. Polarization observables in $p$-d scattering below $30 \mathrm{MeV}$. Phys. Rev. C, 64:024002, 2001.

69. P. Doleschall, W. Grüebler, V. König, P. A. Schmelzbach, F. Sperisen, and B. Jenny. Improved $N N$ interaction tested with $p d$ elastic scattering at $E_{p}=10 \mathrm{MeV}$. Nucl. Phys., A380:72, 1982.

70. G. H. Berthold, A. Stadler, and H. Zankel. Faddeev calculations for low-energy $p-d$ scattering Phys. Rev. C, 41:1365, 1990.

71. D. C. Kocher and T. B. Clegg. Cross sections for proton-deuteron scattering from 1.0 to $10.0 \mathrm{MeV}$. Nucl. Phys., A132:455, 1969.

72. E. Huttel, W. Arnold, H. Berg, H. H. Krause, J. Ulbricht, and G. Clausnitzer. Phase-shift analysis of $p d$ elastic scattering below break-up threshold. Nucl. Phys., A406:435, 1983.

73. A. Kievsky, M. H. Wood, C. R. Brune, B. M. Fisher, H. J. Karwowski, D. S. Leonard, E. J. Ludwig, S. Rosati, and M. Viviani. Evidence for three nucleon force effects in $p$-d elastic scattering. Phys. Rev. C, 63:024005, 2001

74. A. J. Elwyn, R. O. Lane, and Jr. A. Langsdorf. Polarization and differential cross sections in $n$ - $d$ scattering. Phys. Rev., 128:779, 1962

75. K. Sagara, H. Oguri, S. Shimizu, K. Maeda, H. Nakamura, T. Nakashima, and S. Morinobu. Energy dependence of analyzing power $A_{y}$ and cross section for $p+d$ scattering below $18 \mathrm{MeV}$. Phys. Rev. C, 50:576, 1994.

76. W. Grüebler, V. König, P. A. Schmelzbach, F. Sperisen, B. Jenny, R. E. White, F. Seiler, and H. W. Roser Investigation of the three-nucleon system by $p d$ elastic scattering with polarized protons and deuterons. Nucl. Phys., A398:445, 1983.

77. F. Sperisen, W. Grüebler, V. König, P. A. Schmelzbach, K. Elsener, B. Jenny, C. Schweizer, J. Ulbricht, and P. Doleschall. Comparison of a nearly complete $p d$ elastic scattering data set with Faddeev calculations. Nucl. Phys., A422:81, 1984.

78. T. A. Cahill, J. Greenwood, H. Willmes, and D. J. Shadoan. Elastic scattering of protons by deuterium between 15 and $20 \mathrm{MeV}$. Phys. Rev. C, 4:1499, 1971.

79. S. N. Bunker, J. M. Cameron, R. F. Carlson, J. R. Richardson, P. Tomas, W. T. H. van Oers, and J. W. Verba. Differential cross sections and polarizations in elastic $p d$ scattering at medium energies. Nucl. Phys., A113:461, 1968.

80. H. Shimizu, K. Imai, N. Tamura, K. Nisimura, K. Hatanaka, T. Saito, and Y. Koike. Analyzing powers and cross sections in elastic $p d$ scattering at $65 \mathrm{MeV}$. Nucl. Phys., A382:242, 1982

81. K. Sekiguchi, H. Sakai, H. Witała, W. Glöckle, J. Golak, M. Hatano, H. Kamada, H. Kato, Y. Maeda, J. Nishikawa, A. Nogga, T. Ohnishi, H. Okamura, N. Sakamoto, S. Sakoda, Y. Satou, K. Suda, A. Tamii, T. Uesaka, T. Wakasa, and K. Yako. Complete set of precise deuteron analyzing powers at intermediate energies: Comparison with modern nuclear force predictions. Phys. Rev. C, 65:034003, 2002.

82. Owen Chamberlain and Martin O. Stern. Elastic scattering of $190-\mathrm{MeV}$ deuterons by protons. Phys. Rev., 94:666, 1954

83. P. Mermod, J. Blomgren, A. Hildebrand, C. Johansson, J. Klug, M. Österlund, S. Pomp, U. Tippawan, B. Bergenwall, L. Nilsson, N. Olsson, O. Jonsson, A. Prokofiev, P. U. Renberg, P. Nadel-Turonski, Y. Maeda, H. Sakai, and A. Tamii. Evidence of three-body force effects in neutron-deuteron scattering at $95 \mathrm{MeV}$. Phys. Rev. C, 72:061002(R), 2005.

84. K. Hatanaka, N. Matsuoka, H. Sakai, T. Saito, K. Hosono, Y. Koike, M. Kondo, K. Imai, H. Shimizu, T. Ichihara, K. Nisimura, and A. Okihana. Measurements of analyzing powers $A_{y}, A_{x x}, A_{y y}$ and $A_{x z}$ in $d p$ elastic scattering at $E_{d}=56 \mathrm{MeV}$. Nucl. Phys., A426:77, 1984 .

85. S. Nemoto, K. Chmielewski, S. Oryu, and P. U. Sauer. Discrepancy in the cross section minimum of elastic nucleon-deuteron scattering. Phys. Rev. C, 58:2599, 1998.

86. S. Shimizu, K. Sagara, H. Nakamura, K. Maeda, T. Miwa, N. Nishimori, S. Ueno, T. Nakashima, and S. Morinobu. Analyzing powers of $p+d$ scattering below the deuteron breakup threshold. Phys. Rev. C, $52: 1193,1995$. 
87. E. M. Neidel, W. Tornow, D. E. González Trotter, C. R. Howell, A. S. Crowell, R. A. Macri, R. L. Walter, G. J. Weisel, J. Esterline, H. Witała, B. J. Crowe III, R. S. Pedroni, and D. M. Markoff. A new twist to the long-standing three-nucleon analyzing power puzzle. Phys. Lett., B552:29, 2003.

88. J. E. McAninch, L. O. Lamm, and W. Haeberli. Analyzing power in neutron-deuteron elastic scattering at $E_{l a b}^{n}=3 \mathrm{MeV}$. Phys. Rev. C, 50:589, 1994

89. W. Tornow, C. R. Howell, M. Alohali, Z. P. Chen, P. D. Felsher, J. M. Hanly, R. L. Walter, G. Weisel, G. Mertens, I. Slaus, H. Witała, and W. Glöckle. The low-energy neutron-deuteron analyzing power and the ${ }^{3} P_{0,1,2}$ interactions of nucleon-nucleon potentials. Phys. Lett., B257:273, 1991

90. C. R. Howell, W. Tornow, K. Murphy, H. G. Pfützner, M. L. Roberts, Anli Li, P. D. Felsher, R. L. Walter, I. Šlaus, P. A. Treado, and Y. Koike. Comparisons of vector analyzing-power data and calculation for neutron-deuteron elastic scattering from 10 to $14 \mathrm{MeV}$. Few-Body Systems, 2:19, 1987.

91. J. Cub, E. Finckh, H. Frieß, G. Fuchs, K. Gebhardt, K. Geißdörfer, R. Lin, and J. Strate. Analyzing power for elastic $n$ - $d$ scattering at $13 \mathrm{MeV}$. Few-Body Systems, 6:151, 1989.

92. T. Fujita, K. Sagara, K. Shigenaga, K. Tsuruta, T. Yagita, T. Nakashima, N. Nishimori, H. Nakamura, and $\mathrm{H}$. Akiyoshi. Difference between $n d A_{y}$ and $p d A_{y}$ below $16 \mathrm{MeV}$. AIP Conference Proc., 570:709, 2001.

93. G. J. Weisel, W. Tornow, B. J. Crowe III, A. S. Crowell, J. H. Esterline, C. R. Howell, J. H. Kelley, R. A. Macri, R. S. Pedroni, R. L. Walter, and H. Witała. Neutron-deuteron analyzing power data at $19.0 \mathrm{MeV}$. Phys. Rev. C, 81:024003, 2010.

94. H. Witała, D. Hüber, and W. Glöckle. Analyzing power puzzle in low-energy elastic nd scattering. Phys. Rev. C, 49:R14, 1994.

95. A. Kievsky nad M. Viviani and S. Rosati. Cross section, polarization observables, and phase-shift parameters in $p$ - $d$ and $n-d$ elastic scatteing. Phys. Rev. $C, 52: \mathrm{R} 15,1995$.

96. M. H. Wood, C. R. Brune, B. M. Fisher, H. J. Karwowski, D. S. Leonard, E. J. Ludwig, A. Kievsky S. Rosati, and M. Viviani. Low-energy $p$ - $d$ scattering: High-precision data, comparisons with theory, and phase-shift analyses. Phys. Rev. C, 65:034002, 2002.

97. J. C. Faivre, D. Garreta, J. Jungerman, A. Papineau, J. Sura, and A. Tarrats. Polarization in the elastic scattering of protons from deuterons from 11 to $22.7 \mathrm{MeV}$. Nucl. Phys., A127:169, 1969.

98. H. E. Conzett. Tensor analyzing powers in deuteron-proton elastic scattering and the breakup reaction at $45.4 \mathrm{MeV}$. Lect. Note. Phys., 87:477, 1978.

99. A. R. Johnston, W. R. Gibson, J. H. P. C. Megaw, R. J. Griffiths, and R. M. Eisberg. Polarization in the elastic scattering at backward angles of $30 \mathrm{MeV}$ protons from deuterons. Phys. Lett., 19:289, 1965.

100. H. Dobiash, R. Fischer, B. Haesner, H. O. Klages, P. Schwarz, B. Zeitnitz, R. Maschuw, K. Sinram, and K. Wick. Polarization in neutron-deuteron scattering at $30 \mathrm{MeV}$. Phys. Lett., B76:195, 1978

101. J. Zamudio-Cristi, B. E. Bonner, F. P. Brady, J. A. Jungerman, and J. Wang. Measurement of neutrondeuteorn polarization at $35 \mathrm{MeV}$. Phys. Rev. Lett., 31:1009, 1973.

102. W. Tornow, J. H. Esterline, and G. J. Weisel. Energy dependence of the three-nucleon analyzing power puzzle. J. of Phys., G35:125104, 2008.

103. S. Ishikawa. Spin-dependent three-nucleon force effects on nucleon-deuteron scattering. Phys. Rev. C, 75:061002(R), 2007

104. M. Sawada, S. Seki, K. Furuno, Y. Tagishi, Y. Nagashima, J. Shimizu, M. Ishikawa, T. Sugiyama, L. S. Chuang, W. Grüebler, and J. Sanada. Complete set of first-order polarization observables in nucleondeuteron elastic scattering near $20 \mathrm{MeV}$ deuteron energy. Phys. Rev. C, 27:1932, 1983.

105. Y. Koike and J. Haidenbauer. The neutron-deuteron elastic scattering with the Paris potential. Nucl. Phys., A463:365c, 1987.

106. G. Rauprich, H. J. Hähn, M. Karus, P. Nießen, K. R. Nyga, H. Oswald, L. Sydow, H. Paetz gen. Schieck, and Y. Koike. Measurement of $d(\vec{p}, p) d$ elastic scattering at 10.0, 12.0, 14.1 and $16.5 \mathrm{MeV}$ especially for small forward and extreme backward scattering angles. Few-Body Systems, 5:67, 1988.

107. J. Sowinski, D. D. Pun Casavant, and L. D. Knutson. High precision measurement of the analyzing powers for $d-p$ elastic scattering at $E_{d}=10 \mathrm{MeV}$. Nucl. Phys., A464:223, 1987

108. H. Witała, W. Glöckle, L. E. Antonuk, J. Arvieux, D. Bachelier, B. Bonin, A. Boudard, J. M. Cameron, H. W. Fielding, M. Garçon, F. Jourdan, C. Lapointe, W. J. McDonald, J. Pasos, G. Roy, I. The, J. Tinslay, W. Tornow, J. Yonnet, and W. Ziegler. Complete set of deuteron analyzing powers in deuteron-proton elastic scattering: Measurement and realistic potential prediction. Few-Body Systems, 15:67, 1993.

109. G. Rauprich, S. Lemaître, P. Niessen, K. R. Nyga, R. Reckenfelderbäumer, L. Sydow, H. Paetz gen. Schieck, H. Witała, and W. Glöckle. Study of the kinematically complete breakup reaction ${ }^{2} \mathrm{H}(\vec{p}, p p) n$ at $E_{p}=13.0 \mathrm{MeV}$ with polarized protons. Nucl. Phys., A535:313, 1991.

110. J. Strate, K. Geissdörfer, R. Lin, W. Bielmeier, J. Cub, A. Ebneth, E. Finckh, H. Friess, G. Fuchs, K. Gebhardt, and S. Schindler. Differential cross section of the ${ }^{2} \mathrm{H}(n, n n p)$-reaction at $E_{n}=13 \mathrm{MeV}$. Nucl. Phys., A501:51, 1989

111. C. R. Howell, H. R. Setze, W. Tornow, R. T. Braun, W. Glöckle, A. H. Hussein, J. M. Lambert, G. Mertens, C. D. Roper, F. Salinas, I. Šlaus, D. E. González Trotter, B. Vlahović, R. L. Walter, and H. Witała. Implications of the space-star anomaly in nd breakup. Nucl. Phys., A631:692c, 1998.

112. J. Strate, K. Geissdörfer, R. Lin, E. Finckh J. Cub, K. Gebhardt, S. Schindler, H. Witała, W. Glöckle, and T. Cornelius. A three-nucleon break-up experiment in comparison with a Faddeev calculation. J. of Phys., G14:L229, 1988

113. H. R. Setze, C. R. Howell, W. Tornow, R. T. Braun, W. Glöckle, A. H. Hussein, J. M. Lambert, G. Mertens, C. D. Roper, F. Salinas, I. Šlaus, D. E. González Trotter, B. Vlahović, R. L. Walter, and H. Witała. 
Verification of the space-star anomaly in nd breakup. Phys. Lett., B388:229, 1996.

114. J. Kuroś-Źołnierczuk, H. Witała, J. Golak, H. Kamada, A. Nogga, R. Skibinśki, and W. Glöckle. Threenucleon force effects in nucleon induced deuteron breakup. II. comparison to data. Phys. Rev. C, 66:024004, 2002 .

115. S. Kimura, K. Sagara, S. Kuroita, T. Yabe, M. Okamoto, K. Ishibashi, T. Tamura, S. Tanaka, Y. Maeda, and Y. Ooishi. Seach for QFS anomaly in $p d$ breakup reaction below $E_{p}=19 \mathrm{MeV}$. Few-Body Systems, Online First DOI:10.1007/s00601-012-0393-3, 2012.

116. F. D. Correll, R. E. Brown, G. G. Ohlsen, R. A. Hardekopf, N. Jarmie, J. M. Lambert, P. A. Treado, I. Šlaus, P. Schwandt, and P. Doleschall. Study of the reaction $\mathrm{H}(\vec{d}, 2 p) n$ at $16 \mathrm{MeV}$ around collinear configurations. Nucl. Phys., A475:407, 1987.

117. St. Kistryn, E. Stephan, A. Biegun, K. Bodek, A. Deltuva, E. Epelbaum, K. Ermisch, W. Glöckle, J. Golak, N. Kalantar-Nayestanaki, H. Kamada, M. Kiš, B. Kłos, A. Kozela, J. Kuroś Żołnierczuk, M. MahjourShafiei, U. G. Meißner, A. Micherdzińska, A. Nogga, P. U. Sauer, R. Skibiński, R. Sworst, H. Witała, J. Zejma, and W. Zipper. Systematic study of three-nucleon force effects in the cross section of the deuteron-proton breakup at $130 \mathrm{MeV}$. Phys. Rev. C, 72:044006, 2005.

118. St. Kistryn, E. Stephan, B. Kłos, A. Biegun, K. Bodek, I. Ciepal, A. Deltuva, A. C. Fonseca, N. KalantarNayestanaki, M. Kiš, A. Kozela, M. Mahjour-Shafiei, A. Micherdzińska, P. U. Sauer, R. Sworst, J. Zejma, and W. Zipper. Evidence of the Coulomb-force effects in the cross-sections of the deuteron-proton breakup at $130 \mathrm{MeV}$. Phys. Lett., B641:23, 2006.

119. E. Stephan, St. Kistryn, R. Sworst, A. Biegun, K. Bodek, I. Ciepał, A. Deltuva, E. Epelbaum, A. C. Fonseca, J. Golak, N. Kalantar-Nayestanaki, H. Kamada, M. Kiš, B. Kłos, A. Kozela, M. Mahjour-Shafiei, A. Micherdzińska, A. Nogga, R. Skibiński, H. Witała, A. Wrońska, J. Zejma, and W. Zipper. Vector and tensor analyzing powers in deuteron-proton breakup at $130 \mathrm{MeV}$. Phys. Rev. C, 82:014003, 2010.

120. K. Miwa et al. a proposal at J-PARC. 2010. 